LBL --15510

DE83 013753

\title{
PARITY MONCONSERVATION IN ATOMIC THALLIUM: THE MAGNETIC-FIELD EXPERIMENT
}

\author{
Persis Sydney Drel1 \\ (Ph. D. Thesis) \\ Lawrence Berkeley Laboratory \\ University of California \\ Berkeley, California 94720
}

\section{DISCLAMER}

\begin{abstract}
This report was prepared as an account of work sponsored by an agency of the United States Government. Neither the United States Government nor any agency theroof, nor any of their employes, makes any warranty, express or implied, or assumes any legal liability or respona. bility for the accuracy, completeness, or usefulness of any information, apparatus, product, or process disclosed, or represents that its use would not infringe privately owned rights. Refereace berein to any specific commercial product, process, or ervice by trade name, trademark, manufacturer, or otherwise does not neceasurily constitute or imply its endorsement, recommendation, or favoring by the United States Government or any agency thereof. The view and opinions of authors expresed herein do not necessarily state or reflect those of the United Staics Government or any agency thereof.
\end{abstract}

This work was supported by the Director, Office of Energy Research, Office of Basic Energy Sciences, Materials Sciences Division of the U. S. Department of Energy under Contract No. DE-ACO3-76SF00098. 
.

v

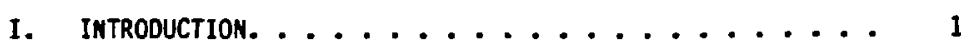

1. The Standard Model. .............. 1

2. Non-Relativistic Reduction of the Heinberg-Salam

Lagrangian: Weak Interactions in Atomic Physics. . 4

a. Bismuth. ................ 8

b. Cesium and Thallium. ............ 12

c. Hydrogen ................. 14

II. PARITY NONCONSERVATION IN ATOMIC THALliUm ....... 17

1. Past Work in Thallium .............. 17

2. Present Work in Thallium. .......... $2 E$.

III. ANALYSIS OF SYSTEMATICS ............. 39

1. Design Considerations ................ 39

2. Misalignments and Imperfect Subtractions. ...... 40

3. Dilutions and 'skgrounds ............ 50

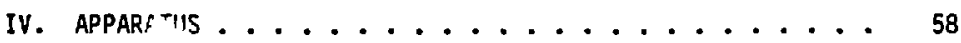

1. Laser ...................... 58

2. Thallium Cell and oven............. 63

3. Electric Field Pulser .............. 81

4. Detection System and Data Acquisition ....... 86

5. Calibration Experiments ............ 91 
v. RESULTS. ............................. 96

1. On-Line Limits .................... 96

2. Circular Polarization Measurements ........ 99

3. $\theta_{y}$ and 700 Gauss Measurements......... 103

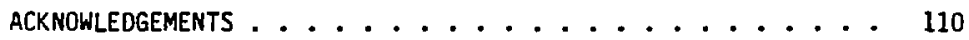
REFERENCES .............................. 111 
 \\ PARITY MONCONSERVATION IN ATOMIC THALLIUM: \\ THE MAGNETIC FIELO EXPERIMENT}

Persis Sydney Drell

(Ph. D. Thesis)

Lawrence Berkeley Laboratory

University of California

Berkeley, California 94720

\section{ABSTRACT}

An experiment underway to measure the parity-nonconserving electric-dipole amplitude in the $6^{2} \mathrm{P}_{1 / 2} \rightarrow 7^{2} \mathrm{P}_{1 / 2}$ transition in atomic thallium is described. Previous measurements ${ }^{31}$ have yielded a value of

$$
\frac{2 \operatorname{Im}\left(\varepsilon_{\text {PNC }}\right)}{M}=+\left(2.8 \pm 0.7_{-0.2}^{+0.3}\right) \times 10^{-3}
$$

The current experiment employs a magnetic field, an electric field, and linearly polarized light from a high-intensity dye-laser oscillator-amplifier system ${ }^{54}$ in an effort to refine the above result. In a large (4 KGauss) magnetic field, an interference between a parity-nonconserving EI and a Stark El amplitude can be observed by pumping the $6^{2} \mathrm{P}_{1 / 2} \rightarrow 7^{2} \mathrm{p}_{1 / 2}$ transition with linearly polarized 293- $n$ m photons and detecting the $7^{2} \mathrm{~S}_{1 / 2} \rightarrow 6^{2} \mathrm{P}_{3 / 2}$ decay

fluorescence at $535 \mathrm{~nm}$. A description of the experiment is presented as well as a detailed analysis of the possible systematic effects that could masquerade as parity nonconservation. Measured limits on the systematics are also presented. 
This report was done with support from the Department of Energy. Any conclusions or opinions expressed in this repor represent solely those of the author[s] and not necessarily those of The Regents of the University of California, the Lawrence Berkeley Laboratory or the Department of Energy.

Reference to a company or product name does not imply approval or recommendation of the product by the University of California or the U.S. Department of Energy to the exclusion of others that may be suitable. 


\section{INTRODUCTION}

\section{The Standard Model}

The concept of using a gauge theory to unify fundamental interactions in physics has enjoyed great popularity in recent years. This is due in large measure to the tremendous success of the Weinberg-Salam model proposed in the late 1960 's 1,2 to unify the weak and the electromagnetic interactions. The model builds on the work of many people ${ }^{3}$ to provide a self-consistent, renormalizable theory that has demonstrated great predictive power.

The primary ingredient of a gauge theory is the requirement of local gauge invariance. This simply means that under transformations on the fields, $\Psi(x)$ of the type

$$
\Psi\left(x_{\mu}\right) \rightarrow \Psi\left(x_{\mu}\right)+i \delta \Psi\left(x_{\mu}\right)
$$

where $\delta=\delta\left(x_{\mu}\right)$ can change arbitrarily from one space time point to the next, the Lagrangian of the system must remain unchanged. One is motivated to require local gauge invariance because the Dirac Lagrangian with the minimal electromagnetic coupling which describes QED, one of the most successful of all theories, is locally gauge invariant under the gauge group $U(1)$. Under the transformation

$$
\Psi(x) \rightarrow e^{i a(x)} \Psi(x)
$$

the Dirac Lagrangian remains unchanged.

The next ingredients of a gauge theory of the weak and electromagnetic interactions are spontaneous symmetry breaking and the Higgs mechanism whereby one can give the gauge quanta mass and still have a renormalizable theory. ${ }^{4}$ Massive gauge quanta are essential to any theory incorporating the weak interactions since the weak force 
is short ranged. In the Weinberg-Salam mode1, the gauge group chosen is $S U(2) \times U(1)$. This results in three massive gauge quanta, $W^{ \pm}$and $z^{0}$, corresponding to the short range weak interactions, and the familiar massless photon of QED. Other models use larger gauge groups and have correspondingly more gauge quanta. While experimental evidence does not exclude some of these models, nothing favors them either.

When one then introduces the matter fields of quarks and leptons, there is considerable freedom in choosing how they will transform under transformations of the gauge group. One chooses to couple them in such a way as to reproduce known phenomenology, and it is in the choice of quark and lepton couplings, that the parity nonconserving nature of the weak interactions is inserted. The Weinberg-Salam choice is: The left handed components of the quarks and leptons are in SU(2) isodoublets while the right handed components are in isosinglets under $\mathrm{SU}(2)$. This multiplet asssignment can be written as:

$$
\left[\begin{array}{l}
v_{e} \\
e^{-}
\end{array}\right] L, e_{R}^{-},\left[\begin{array}{l}
v_{H} \\
H^{-}
\end{array}\right] L, \mu_{R}^{-}, \ldots\left[\begin{array}{l}
u \\
d_{c}
\end{array}\right] L, u_{R}, d_{C_{R}},\left[s_{c}\right] L, c_{R}, s_{C_{R}}, \ldots
$$

When the couplings of the matter fields have been fixed to conform to the known electromagnetic and charged current weak interaction phenomenology, one is left with one free parameter of the theory, $\theta_{W}$, which characterizes the neutral weak interactions.

The amount of experimental evidence supporting the Weinberg-Salam model is impressive. That tests of charged current couplings agree 
with the model is not surprising since the couplings were chosen to agree with existing charged current data, but the model has also proved successful in predicting previously unobserved neutral current effects. Neutral currents were first observed and studied in high energy neutrino experiments ${ }^{5}$ in the reaction

$$
v_{\mu}+N \rightarrow v_{\mu}+N^{\prime} \text {. }
$$

All of the experimental data are consistent with the predictions of the Weinberg-Salam mode $l^{6}$ with a value of

$$
\sin ^{2} \theta_{W}^{\exp }=0.216 \pm 0.010
$$

when $O(\alpha)$ corrections are taken into account. ${ }^{7}$ Evidence also supports the Weinberg-Salam form of the interaction

$$
e^{-}+N^{-}+e^{-}
$$

for electron-quark couplings in results from a beautiful experiment at SLAC. ${ }^{8}$ A parity nonconserving asymmetry in the inelastic scattering of polarized electrons off of a deuterium target was observed and found consistent with the Weinberg-Salam predictions for

$$
\sin ^{2} \theta_{W}=0.215 \pm .015
$$

when $0(\alpha)$ corrections are taken into account. 9 Early results from atomic physics measurements refuted the weinberg-Salam predictions for eN couplings, but these results now are also consistent with the theory for the most part. (These experiments will be discussed in more detail subsequently.)

Further confirming evidence is beginning to come in with the observation ${ }^{10}$ of an asymmetry in the scattering

$$
\mathrm{e}^{+}+\mathrm{e}^{-} \rightarrow \mu^{+}+\mu^{-} \text {. }
$$

Most spectacular, however, are the preliminary reports of direct 
observation of the bosons that mediate the charged weak interactions. 11 Studies of direct $W$ and $Z$ production should revea? information not only on the first order weak interaction effects, but provide tests of higher order corrections in the Weinberg-Salam model. This is important since the ability to calculate higher order terms is one of the great attractions of a gauge theory. Perhaps future experiments will also give a clue as to the existence of the Higgs particle. This is a scalar particle whose existence is necessary in order to incorporate masses into the gauge theory and still retain renormalizability. It is a particle for which there is no evidence so $f a r$, and experimental verification of its existence would be very exciting.

2. Non-Relativistic Reduction of the Heinberg-Salam Lagrangian: Weak Interactions in Atomic Physics

The experiment described herein is an attempt to further understand the nature of the electron nucleon coupling due to weak neutral currents by examining an atomic system for evidence of parity nonconservation. Figure I-1 shows the interaction schematically in terms of Feynman diagrams where the Weinberg-Salam model predicts values for the vertex couplings. (Any parity violation due to nuclear charged current interactions is $O(a)$ smaller and hence negligible). ${ }^{33}$ If it is assumed that the neutral currents contain only vector and axial vector components ${ }^{12}$ then the currents take the form

$$
\begin{aligned}
& J_{e}^{\mu}=V_{e}^{\mu}+A_{e}^{\mu} \\
& J_{N}^{\mu}=V_{N}^{\mu}+A_{N}^{\mu}
\end{aligned}
$$

as the sum of vector and axial vector portions. The total amplicude 

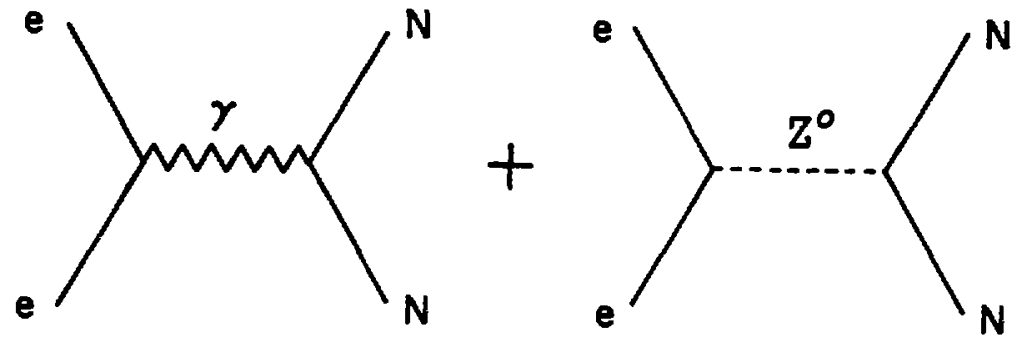

XBL 833-8844

Fig. I-1. Feynmman diagrams for photon $(y)$ and neutral vector boson $\left(z^{0}\right)$ exchange between electron and nucleon. 
$J_{\mu} J^{U}$ can be written as a sum of scalar and pseudoscalar parts:

$$
J_{e}^{\mu_{N}} J_{\mu}=\left(V_{e} V_{N}+A_{e} A_{N}\right)+\left(V_{e} A_{N}+V_{N} A_{e}\right) \text {. }
$$

The scalar terms are unobservable but the two pseudoscalar terms can be observed. In the Weinberg-Salam model, (I.10) is predicted to be:

$j_{e}^{\nu_{j} j_{p}}=\frac{G_{F}}{2 \sqrt{2}} \bar{u}_{e}^{\prime} \gamma_{\lambda}\left(1-4 \sin ^{2} \theta_{W}-r^{5}\right) u_{e} \cdot \bar{u}_{p}^{\prime} \gamma^{\lambda}\left(1-4 \sin ^{2} \theta_{W}-1.2 \gamma^{5}\right) u_{p}$

for protons and

$$
J_{e}^{j} J_{n_{u}}=-\frac{G_{F}}{2 \sqrt{2}} \bar{u}_{e}^{\prime} \gamma_{\lambda}\left(1-4 \sin ^{2} \theta_{W}-\gamma^{5}\right) u_{e} \cdot \bar{u}_{n}^{\prime} \gamma^{\lambda}\left(1-1.2 \gamma^{5}\right) u_{n}
$$

for neutrons. 4 For a nucleus with $Z$ protons and $N$ neutrons, (1.11) and (I.12) can be combined and in the limit of a non-relativistic point nucleus one finds

$$
\begin{gathered}
V_{P N C}=\frac{G_{F}}{4 \sqrt{2 m_{e} c}} Q_{W}\left[\vec{\sigma} \cdot \vec{p} \delta^{3}(\vec{r})+\delta^{3}(\vec{r}) \vec{\sigma} \cdot \vec{p}\right] \\
Q_{W}=\left(1-4 \sin ^{2} \theta_{W}\right) z-N \\
V_{P N C}=\frac{1.2 G_{F}}{4 \sqrt{2 m_{e} c}}\left(1-4 \sin ^{2} \theta_{W}\right)\left[\vec{\sigma} \cdot \vec{\sigma}_{n} \vec{\sigma} \cdot \vec{p} \delta^{3}(\vec{r})\right. \\
\left.+s^{3}(\vec{r}) \vec{\sigma} \cdot \vec{\sigma}_{n} \vec{\sigma} \cdot p\right]
\end{gathered}
$$

where $V_{\text {PNC }}$ and $V_{\text {PNC }}$ are the effective potentials induced by the $V_{N} A_{e}$ and $V_{e} A_{N}$ terms respectively. ${ }^{4}$ The vectors $\vec{\sigma}, \vec{p}$, and $\vec{r}$ refer to the electron whereas $\vec{\sigma}_{n}$ refers to the nucleons.

The atomic physics experiments performed on heayy atoms are sensitive to $V_{P N C}$ only. This is because, relative to $V_{P N C}$ ', $V_{P N C}$ has an extra factor of $Z$ since the nucleons contribute coherently. In $V_{\text {PKC }}$ the nucleons add proportional to spin, and 
when one sums over the nucleons terms cancel in pairs leaving at most one unpaired nucleon to contribute. These heavy atom experiments derive great benefit from the approximately $z^{3}$ dependence in $V_{\text {PNC }}$. The other two factors come from the density of the valence electron at the nucleus and from the velocity of the electron near the nucleus, both of which are approximately proportional to $z^{13}$ Experiments in hydrogen are sensitive to effects of both $V_{P N C}$ and $V_{\text {PNC }}$.

The effective potentials due to neutral currents must be combined with the familiar electromagnetic atomic hamiltonian to give the correct total hamiltonian for the system:

$$
H_{\text {TOTAL }}=H_{E M}+V_{\text {PNC }} \text {. }
$$

This new total hamiltonian no longer commutes with the parity operator due to the presence of the pseudoscalar term, $V_{P N C}$, so the atomic eigenstates will no longer be eigenstates of the parity operator. One expects to see transitions that are forbidden by electromagnetic parity selection rules, now allowed to order $G_{F}^{2}$. Such effects would be extremely difficult to see, but if a transition can proceed by an electromagnetic and a weak amplitude, interference terms, linezr in $G_{F}$, will appear and are, in fact, observable. 14,15

Consider an electromagnetic transition between two states of the same nominal parity, taking into account the effects of "PNC to first order in perturbation theory $\left(V_{F N C} \ll H_{E M}\right)$ :

$$
\left\langle\bar{u}_{2}\left|0_{E M}\right| \bar{u}_{1}\right\rangle=\left\langle u_{2}\left|o_{E M}\right| u_{1}\right\rangle+\sum_{n}^{\left\langle u_{2}\left|o_{E M}\right| v_{n}\right\rangle \mid\left\langle v_{n}\left|v_{P N C}\right| u_{1}\right\rangle} \frac{E_{u_{1}}-E_{v_{n}}}{E_{n}}+1 \leftrightarrow 2
$$


where $i$ and $v$ are states of opposite parity. For a transition that is nominally magnetic dipole, one gets:

$$
\left\langle\overline{u_{2}}\left|O_{E M}\right| \overline{u_{1}}\right\rangle=M 1+i E 1_{P N C} .
$$

Under a parity transformation of the system, this transition anplitude becomes $M 1$ - $i E 1_{\text {PNC }}$ so that one can see an interference by looking at an asymetry in the light absorbed on the transition:

$$
\Delta_{\text {PMC }}=\frac{W(R)-W(L)}{W(R)+W(L)}=\frac{2 \operatorname{Im}\left(E 1_{P N C}\right)}{W I},
$$

where $R$ and $L$ refer to two experimental configurations related by a parity transformation. Attempts to measure $\triangle_{\text {PNC }}$ in atoms have fnllowed three independent courses depending on the atomic system used. These different experiments will now be briefly discussed, and theoretical and experimental results presented.

\section{a. Bismuth}

The first experiments to measure $\Delta_{\text {PNC }}$ were done in Bismuth. 16-21 Attempts were made to measure a very small optical rotation in Bismuth vapor due to the interference of an allowed $M 1$ with the parity nonconserving El amplitude. Linearly polarized light near resonance passes through a bismuth vapor cell and the plane of polarization rotates slightly because the MI-El interference results in a difference in the refractive index for right and left circularly polarized light. The angle of rotation is given by

$$
\sigma_{\text {PNC }}=\frac{4 \pi(n-1) L}{\lambda} \frac{-i E 1_{\text {PNC }}}{\text { MI }}
$$

where $n$ is the index of refraction, $\lambda$ the wavelength, and $L$ is the path length. Typical estimates of the rotation are 
The leading term in the transition probability is

$$
\begin{cases}B_{2}{ }^{2} \sin ^{2} \theta & \Delta m_{F}=0 \\ B_{2}{ }^{2} \cos ^{2} \theta & \Delta m_{F}= \pm 1 .\end{cases}
$$

Using IV.9 and recalling that

$$
B_{2}=B\left(E_{\text {oy }}+\Delta E_{y}\right)
$$

where $E_{o y}$ is the reversing and $\Delta E_{y}$ the non-reversing $y$ component of the electric field, then

$$
Y_{E}=\frac{S\left(E_{+}\right)-S\left(E_{-}\right)}{S\left(E^{+}\right)+S\left(E_{-}\right)}=\frac{2 \Delta E_{y}}{E_{D y}} .
$$

The quantity $\gamma_{E}$ is computed separately for the two regions. The sizes of the electric field for $\mathrm{E}^{+}$and $\mathrm{E}-$ can be independently adjusted for the regions individually in order to minimize $\gamma_{E}$. It is possible during a run to keep

$$
\left|\Delta E_{y}\right|<5 \times 10^{-4}\left|E_{o y}\right|
$$

for each region. This value is combined with other data to put a limit on the $\mathrm{B}_{2}{ }^{2} \mathrm{\theta}_{\mathrm{x}}$ interference term which gives an asymmetry of

$$
{ }^{\theta} x \text { alse }=\frac{4 \Delta E_{y^{\theta} x}}{E_{o y}} \text {. }
$$

(Note that $\Delta E_{y}$ does not enter into the asymmetries induced by the other interference terms containing a factor of $\beta_{2}$ except for effects which are of third order and thus negligible.)

Now consider $\gamma_{U V}$ which is also measured on-line. Recall that

$$
Y_{U V}=\frac{S(U V+)-S(U V-)}{S(U V+)+S(U V-)}
$$

where now more than the leading term in (V.1) must be kept in computing $r_{U V}$. Returning to the expressions for the total 


\begin{tabular}{|c|c|c|}
\hline Transition & $\begin{array}{r}R=\operatorname{Im} \frac{E T_{\text {PNC }}}{N I} \\
\text { (units of } 10^{-8} \text { ) }\end{array}$ & Group \\
\hline $\mathrm{J}=\underset{ }{(876 \mathrm{~nm})}=3 / 2$ & $\begin{array}{r}0.2 \pm 1.5 \\
-10.4 \pm 1.7\end{array}$ & $\begin{array}{l}\text { Seattle } 197716 \\
\text { Seattle } 1981^{21}\end{array}$ \\
\hline $\mathrm{J}=\underset{(648 \mathrm{~nm})}{3 / 2 \rightarrow \mathrm{J}^{\prime}}=5 / 2$ & $\begin{aligned} 2.7 & \pm 4.7 \\
-20 & \pm 5.5 \\
-20.6 & \pm 3.2 \\
-2.3 & \pm 1.3 \\
-10.7 & \pm 1.5\end{aligned}$ & $\begin{array}{l}\text { Oxford } 1977^{17} \\
\text { Novisibirsk } 197818 \\
\text { Novisibirsk } 197919 \\
\text { Moscow } 1980^{20} \\
\text { Oxford } 1980^{26} \\
\text { (unpublished) }\end{array}$ \\
\hline
\end{tabular}
Fig. I-2(a). Experimental results for $R=\frac{I m\left(E I_{P N C}\right)}{M I}$ from optical rota-
tion experiments in bismuth. 


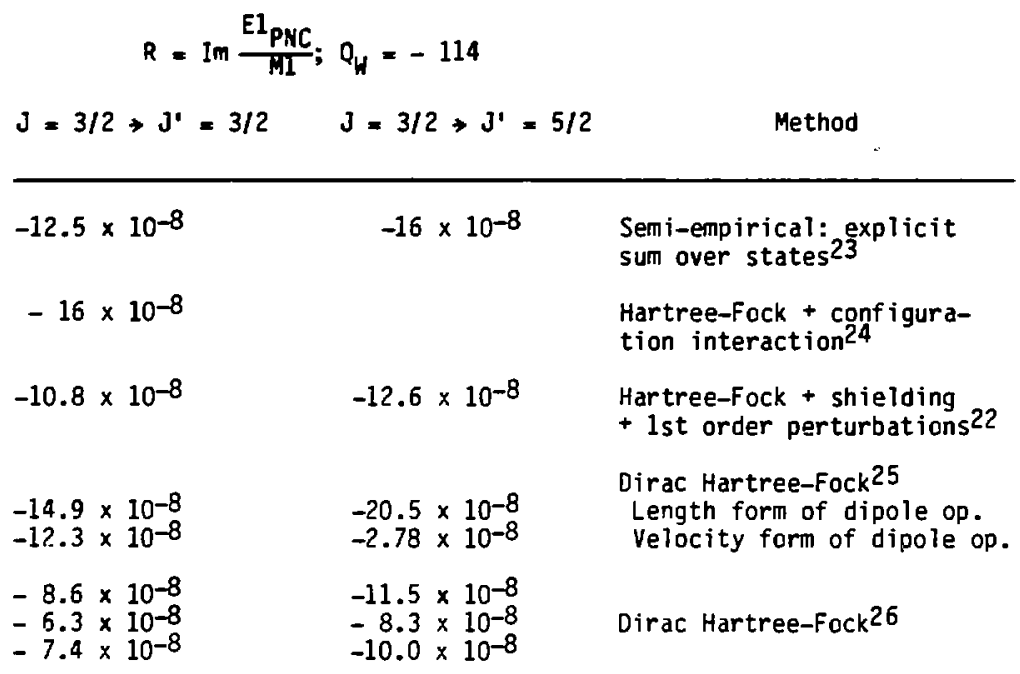

Fig. $I-2(b)$; Theoretical values for $R=\frac{I m\left(E I_{P N C}\right)}{M I}$ in bismuth with $\sin ^{2} \theta_{W}=.21$. 
calculations and discuss their possible shortcomings. They suggest that there are three possible sources of difficulty in bismuth:

(i) shielding effects ( $i$ ) exchange effects ( $i i$ ) complications due to the breakdown of $j \mathrm{j}$ coupling. Both conclude more work is necessary to clarify both the experimental and theoretical situation.

b. Cesium and Thallium

In both cesium and thallium one works on a highly forbidden M1 transition. A laser pumps a transition $\left(6^{2} \mathrm{~S}_{1 / 2} \rightarrow 7^{2} \mathrm{~S}_{1 / 2}\right.$ in Cs, $6^{2} \mathrm{P}_{1 / 2} \rightarrow 7^{2} \mathrm{P}_{1 / 2}$ in $\left.\mathrm{TI}\right)$ in the presence of an external electric field which induces a parity conserving Stark El amplitude. The interference between $E 1_{\text {Stark }}$ and $E 1_{\text {PNC }}$ is then observed by looking at the decay fluorescence from the excited state as the handedness or parity of the system is changed. The experimentaliy observed asymmetries are

$$
{ }_{\text {PNC }}-.3-1 \times 10^{-5}
$$

Experifiients in cesium are being conducted in several tabs. $28-30$ The first observation of parity nonconservation in atomic cesium has recently been published, 29 and the results are in good agreement with theory with $\theta_{W}=.21$ (see Fig. 1-3). (Radiative corrections lower the value of $\sin ^{2} \theta_{W}$ for atomic energies when compared with the value ascertained from neutrino scattering.) Other experiments have made preliminary measurements of Stark amplitudes but are not yet sensitive enough to measure $E 1_{\text {PNC }}$. Results consistent with theory have been published measuring PNC in thallium ${ }^{31}$ and efforts are continuing to make the results more precise. 


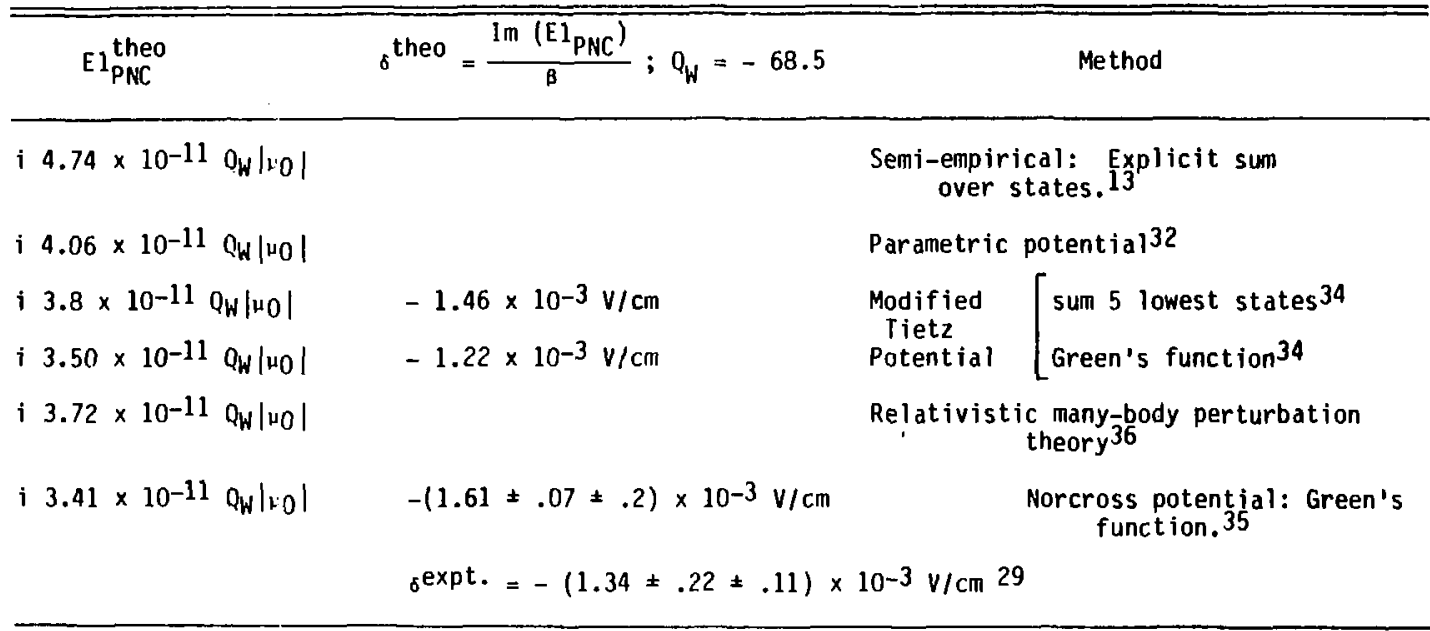

Fig. 1-3. Theoretical values for the parity nonconserving El amplitude in cesium. For comparison with the experimental value, $\delta$ theo $=\frac{\operatorname{Im}\left(E 1_{P N C}\right)}{\beta}$ is given. 
Theoretically both cesium and thallium are considerably simpler than bismuth. Very diverse calculational methods yield consistent predictions for values of the parity nonconserving transition amplitude. The lower lying states of both atcins are He: $:$ descrited in a one-electron central field approximation and semi-empirical calculations can be expected to yield accurate results. Sophisticated many body perturbation theory calculations are consistent with parametric potential calculations. A summary of theoretical predictions for $E 1_{\text {PNC }}$ on cesium is given in Fig. I-3 along with the experimental result for comparison. Results from thallium calculations are given in Fig. II-3. The abundance of spectroscopic data for both atoms provides additional consistency checks for the atomic calculations and the agreement between theory and experiment is good. Confidence in the level of understanding of the atomic physics in cesium is such that attempts are being made to refine the experimental results to the few percent level where it is hoped that effects due to the $V_{e} A_{N}$ term (I.13) can be seen. At this level, radiative corrections might also be in evidence. ${ }^{36}$

\section{c. Hydrogen}

The experiments being pursued in hydrogen are still several orders of magnitude in sensitivity away from being able to test predictions of neutral current effects. Predicted asymmetries in hydrogen are much smaller than in heavy atoms because of the absence of any $z^{3}$ enhancement. Some advantage can be regained by working on the 25-2P states which at zero magnetic field are separated only by the Lamb shift. At a finite magnetic field, the energy denominator in Eq. 
(I.15) can become as small as $100 \mathrm{thz}$ at a 2S-2P level crossing. The experimental methods used are described in greater detail elsewhere. ${ }^{12,37}$ Very briefly, a beam of hydrogen atoms is prepared in a component of the metastable $2^{2} \mathrm{~S}_{1 / 2}$ state. Transitions are induced in a microwave cavity to another component of the $2^{2} \mathrm{~S}_{1 / 2}$ state with a magnetic field tuned to a $2 \mathrm{~S}-2 \mathrm{P}$ Tevel crossing in order to maximize the PNC matrix element. A small external electric field Stark mixes states to enhance the signal and results in an interference between the Stark assisted $E 1$ amplitude and $E 1_{\text {PNC }}$. The number of atoms that have made the microwave transition is measured downstream and an asymmetry in that number, as the parity of the interaction region is changed by reversing $E, B$ or $E$, is sought. The expected size of the asymmetry is $10^{-7}-10^{-6}$ and the signals are extremely small.

The great advantage of hydrogen is the precision and reliability with which calculations can be done. Also, the hydrogen experiments are sensitive to both $V_{N} A_{e}$ and the $V_{e} A_{N}$ term which is inaccessiible at this point to the heavy atom experiments. In fact, all the current hydrogen experiments are attempting to measure $V_{e} A_{N}$ first.

The most recent progress repcrt is from the Michigan group. ${ }^{38}$ They put an upper 1 imit of $2.5 \times 10^{-3}$ on a PNC asymmetry which trans Tates into a limit of

$$
C_{2 p}=.62\left(1-4 \sin ^{2} \theta_{W}\right)<620
$$

for the coefficient of the $V_{e} A_{N}$ term in (I.13). The expected value in the Weinberg-Salam model is $C_{2 p}=.099$ for $\sin ^{2} \theta_{W}=.21$. The experimental difficulties to be overcome before this measurement can be 
improved are formidable. Motional electric fields and misalignments must be smaller than $10^{-4}-5 \times 10^{-6} \mathrm{~V} / \mathrm{cm}$ for the various experiments and field reversals must be good to a part in $10^{-4} .12$ It is likely to be some time before such technical acheivements will be possible.

We shall now return to a description of continuing efforts to refine the measuremerit of $E 1_{\text {PNC }}$ in atomic thallium. The experiment and its current status will be described in detail in subsequent chapters. 


\section{PARITY NONCONSERVATION IN ATOMIC THALLIUM}

1. Past Work in Thalium

For the past eight years, Eugene Commins and his collaborators at Berkeley have conducted a research program to determine whether or not parity is conserved in atomic thallium. These efforts have yielded calculations of the thallium wavefunctions and theoretical predictions as well as experimental values for various transition amplitudes in thallium, including the parity nonconserving (PNC) amplitude. Results are summarized in Fig. II-2 and II-3, and are more fully explained in the references quoted there. The experiment described here represents an effort to improve the precision of the parity measurement with a different technique that has independent systematic errors associated with it.

The thallium atom has 81 electrons, 80 of them in closed shells with one additional $6 \mathrm{P}$ electron in the ground state configuration. The ground state and lower lying excited states are well described in terms of a single valence electron outside of a spherically symetric closed core. As a result, calculations of wavefunctions and transition amplitudes can be made with confidence due to their relative simplicity, despite the large $z$. The availability of large quantities of spectroscopic data provides an excellent test of the reliability of these calculations.

In the calculations on thallium done by Neuffer and Commins, 39 valence electron wavefunctions were generated as numerical solutions to the Dirac equation in a modified Tietz central potential. These wavefunctions were then used to calculate transition amplitudes, 
energy levels, and fine and hyperfine structure splittings for low lying states. Other methods of generating wavefunctions have also been used by different groups $13,36,40$ to calculate transition elements and their results are in good agreement with those of Neurfer and Commins. In particular, calculations of hyperfine structure splittings are important since they test the value of the wavefunction at the origin, and the generally good agre ment between calculated and experimental numbers gives one confidence that the lestaviour nf the wavefunction there is understood. Since it is a contar. ". cential, the PNC amplitudie depends on the wavefunction at the jigin. Unfortunately, it also depends on the derivative of the wavefunction at the origin (see Eq. I.13), a quantity none of the spectroscopic data are sensitive to.

In the current experiment, the transition amplitudes of greatest interest are the PNC amplitude of course, and the Stark amplitudes, referred to as a and $B$, which enter when an electric field is externally applied to the atoms. The particular transition of interest is from the ground state $\sigma^{2} p_{1 / 2}$ to the $7^{2} p_{1 / 2}$ state (Fig. II-1). Nominally this is a forbidden magnetic dipole transition with measured amplitude of 43

$$
M=(-2.1 \pm 0.3) \times 10^{-5}\left|\frac{e \hbar}{2 m e^{c}}\right| .
$$

Parity nonconservation causes admixing of the $6^{2} \mathrm{P}_{1 / 2}$ and $7^{2} \mathrm{P}_{1 / 2}$ states with nearby $n^{2} \mathrm{~S}_{1 / 2}$ states and admits an 


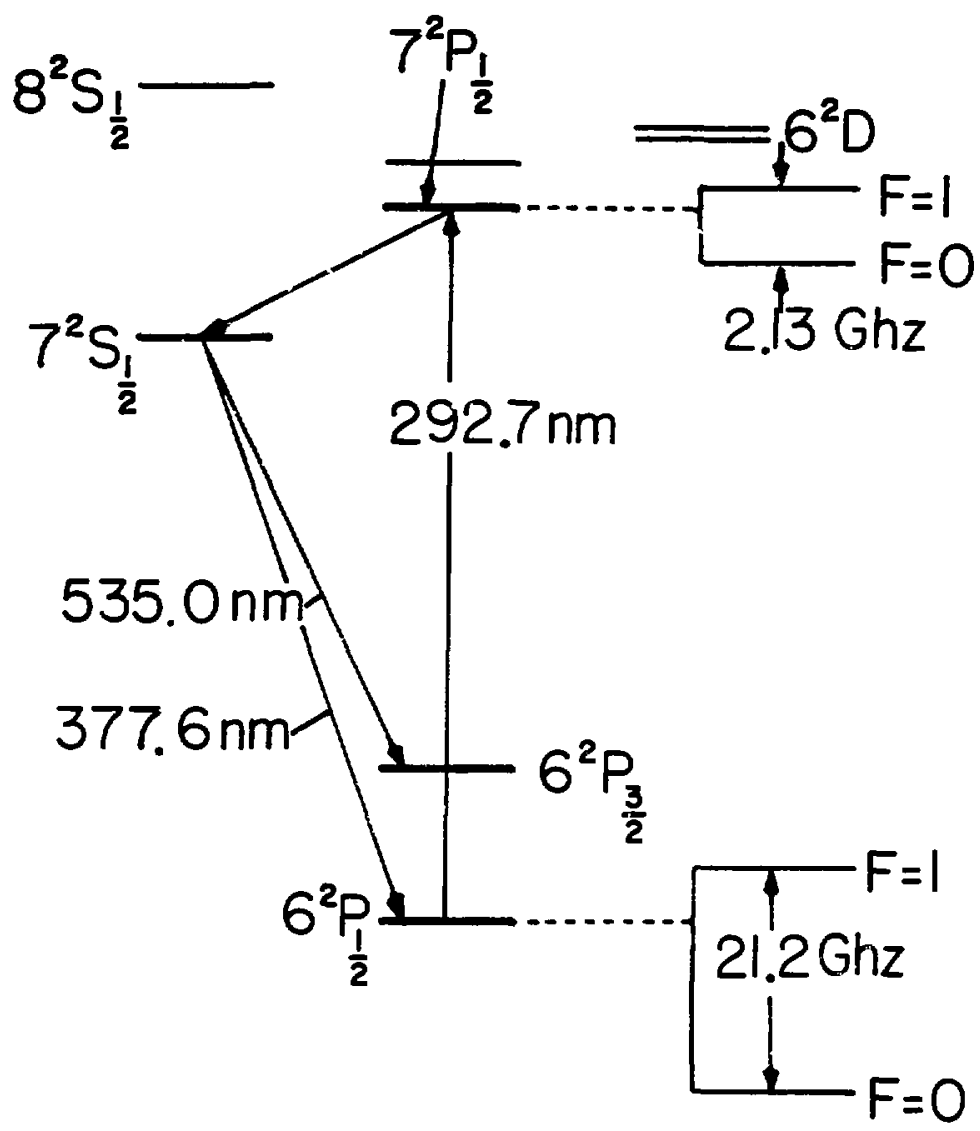

XBL $833-8845$

Fig. Il-1. Low-lying energy levels of Tl (not to scale). The hyperfine splittings of $6^{2} \mathrm{p}_{1 / 2}, 7^{2} \mathrm{p}_{1 / 2}$ states are
shown. 41,42 
additional electric dipole transition amplitude. In first order perturbation theory the perturbed states can be written

$$
\begin{aligned}
& \overline{\left|6^{2} P_{1 / 2}\right\rangle}=\left|6^{2} P_{1 / 2}\right\rangle+\sum_{n S} \frac{\left\langle n S\left|v_{P_{N C}}\right| 6^{2} P_{1 / 2}\right\rangle}{E_{6 P}-E_{n S}}|n S\rangle \\
& \left|7^{2} P_{1 / 2}\right\rangle=\left|7^{2} P_{1 / 2}\right\rangle+\sum_{n S} \frac{\left\langle n S\left|v_{P N C}\right| 7^{2} P_{1 / 2}\right\rangle}{E_{7 P}-E_{n S}}|n S\rangle
\end{aligned}
$$

where $V_{P N C}$ is the PNC effective potential described in Eq. (I.13). The electric dipole amplitude between the states is then

$$
\begin{aligned}
& \left\langle\overline{7^{2} P_{1 / 2}}\right| E 1 \overline{\left|6^{2} P_{1 / 2}\right\rangle}=\sum_{n S} \frac{\left\langle 7^{2} P_{1 / 2}|E l| n S\right\rangle\left\langle n S\left|V_{P N C}\right| 6^{2} P_{1 / 2}\right\rangle}{E_{6 P}-E_{n S}} \\
& +\sum_{n S} \frac{\left\langle 7^{2} p_{1 / 2}\left|V_{P N C}^{+}\right| n S>\left\langle n S|E 1| \sigma^{2} p_{1 / 2}\right\rangle\right.}{E_{7 p}-E_{n S}} .
\end{aligned}
$$

Many of the El matrix elements, $\langle n P|E 1| n S\rangle$, have been experimentally determined by measurements of excited state lifetimes (see Fig. II-2), but the PNC matrix element must be calculated. Neuffer and Commins predict $^{39}$

$$
\begin{aligned}
E_{\text {PNC, theo }} & =\left\langle 7^{2} P_{1 / 2}, m_{j}=-\frac{1}{2}|E 1| 6^{2} P_{1 / 2}, m_{j}=-\frac{1}{2}\right\rangle \\
& =+1.93 \times 10^{-10} i Q_{w}\left|\frac{e \hat{h}}{2 m_{e} c}\right|, Q_{w}=2\left(1-4 \sin ^{2} \theta_{w}\right\rangle-N \\
& =-2.12 \times 10^{-8} i\left|\frac{e h}{2 m_{e}^{c}}\right|
\end{aligned}
$$


A coefficient $\left(10^{7} \mathrm{sec}^{-1}\right)$

Transition Theory (Ref. 39)

Experiment (Ref. 45 unless otherwide indicated)

\begin{tabular}{lll}
\hline $7^{2} s_{1 / 2}-6^{2} p_{1 / 2}$ & 5.78 & $6.25 \pm 0.31$ \\
$8^{2} s_{1 / 2}-6^{2} p_{1 / 2}$ & 1.75 & $1.78 \pm 0.16$ \\
$9^{2} s_{1 / 2}-6^{2} p_{1 / 2}$ & 0.777 & $0.78 \pm 0.10$ \\
$11^{2} s_{1 / 2}-6^{2} p_{1 / 2}$ & 0.244 & $0.31 \neq 0.06$ \\
$7^{2} s_{1 / 2}-6^{2} p_{3 / 2}$ & 8.30 & $7.05 \pm 0.32$ \\
$8^{2} s_{1 / 2}-6^{2} p_{3 / 2}$ & 2.30 & $1.73 \pm 0.18$ \\
$9^{2} s_{1 / 2}-6^{2} p_{3 / 2}$ & 1.01 & $0.80 \pm 0.08$ \\
$10^{2} s_{1 / 2}-6^{2} p_{3 / 2}$ & 0.534 & $0.57 \pm 0.06$ \\
$6^{2} D_{3 / 2}-6^{2} p_{1 / 2}$ & 15.04 & $12.6 \pm 1.0$ \\
$7^{2} D_{3 / 2}-6^{2} p_{1 / 2}$ & 6.39 & $4.4 \pm 0.5$ \\
$8^{2} D_{3 / 2}-6^{2} p_{1 / 2}$ & 3.19 & $1.89 \pm 0.3$ \\
$9^{2} D_{3 / 2}-6^{2} p_{1 / 2}$ & 1.82 & $0.98 \pm 0.22$ \\
$10^{2} D_{3 / 2}-6^{2} p_{1 / 2}$ & 1.14 & $0.58 \pm 0.15$ \\
$6^{2} D_{3 / 2}-6^{2} p_{3 / 2}$ & 16.3 & $2.20 \pm 0.23$ \\
$7^{2} D_{3 / 2}-6^{2} p_{3 / 2}$ & 2.88 & $0.76 \neq 0.08$ \\
$8^{2} D_{3 / 2}-6^{2} p_{3 / 2}$ & 1.01 & $0.37 \pm 0.04$ \\
$9^{2} D_{3 / 2}-6^{2} p_{3 / 2}$ & 0.498 & $0.19 \pm 0.02$ \\
$6^{2} D_{5 / 2}-6^{2} p_{3 / 2}$ & 0.279 & $12.4 \pm 1.5$ \\
& & $F 19.11-2$
\end{tabular}

Fig. II-2 continued 


\section{A coefficient $\left(10^{7} \mathrm{sec}^{-1}\right)$}

Transition

Theory (Ref. 39)

Experiment (Ref. 45

uniess otherwide indicated)

$\begin{array}{lll}7^{2} \mathrm{D}_{5 / 2}-6^{2} \mathrm{P}_{3 / 2} & 6.06 & 4.2 \pm 0.5 \\ 8^{2} \mathrm{D}_{5 / 2}-6^{2} \mathrm{P}_{3 / 2} & 2.96 & 1.7 \pm 0.2 \\ 7^{2} \mathrm{~S}_{1 / 2}-7^{2} \mathrm{p}_{1 / 2} & 1.88 & 1.71 \pm 0.07 * \\ 6^{2} \mathrm{D}_{3 / 2}-7^{2} \mathrm{p}_{1 / 2} & 0.0479 & 0.0597 \pm 0.0078 *\end{array}$

*These experimental values are from reference 44 .

Fig. II-2. Comparison of theoretical and experimental vaiues for allowed El transition rates. The radial matrix elements determined from these $A$ coefficients enter in the calculation of $a$ and $B$ as well as EPNC. Only transitions for which there is experimental data have been listed here. For a more complete summary see ref. 39 . 
for $\sin ^{2} \theta_{w}=.21$. A more recent calculation using relativistic many-body perturbation theory $y^{36}$ predicts a somewhat smaller value of

$$
\varepsilon_{\text {PNC, theo }}=(1.51 \pm 0.07) \times 10^{-10} i Q_{W}\left|\frac{\text { eh }}{2 m e^{c}}\right| \text {. }
$$

Several other authors have calculated this quantity and the results are summarized in Fig. II-3. Experimentally, the quantity $2 I m\left(\varepsilon_{\text {PNC }}\right) / M$ has been determined at Berkeley to be ${ }^{31}$

$$
2 \operatorname{Im}\left(\varepsilon_{\mathrm{PNC}}\right) / M=+\left(2.8 \pm 0.7_{-0.2}^{+0.3}\right) \times 10^{-3} .
$$

A direct measurement of the interference $2 \operatorname{Im}\left(\varepsilon_{\text {PNC }}\right) / M$, where $M$ is a forbidden magnetic dipole transition amplitude, would be an attractive experiment because of the size of the asymmetry (see I.16 and I.17). Such an experiment is not possible since light scattering and other backgrounds would obscure the resonance. Instead, a technique suggested by Bouchiat and Bouchiat ${ }^{13}$ is used where an external electric field of a $100-300 \mathrm{~V} / \mathrm{cm}$ is applied to the thallium vapor and the Stark signal is observed. The electric field admixes $\mathrm{nS}_{1 / 2}$ and $\mathrm{nD}_{3 / 2}$ states with the $6^{2} \mathrm{p}_{1 / 2}$ and $7^{2} \mathrm{p}_{1 / 2}$ states, an electric dipole transition is then allowed, and the interference between the Stark induced electric dipole amplitude and the PNC electric dipole amplitude yields an asymetry, $2 \operatorname{Im}\left(\varepsilon_{\mathrm{PNC}}\right) / \varepsilon_{\text {Stark }}$, which is observed. Unfortunately, one loses two orders of magnitude in the size of the asymmetry with this technique, since 


E1 $1_{P N C}^{\text {theo }}\left(6^{2} P_{1 / 2} \rightarrow 7^{2} P_{1 / 2}\right) \quad \quad s^{\text {theo }}=\frac{2 I m\left(E_{P N C}\right)}{M} ; Q_{W}=-110 \quad$ Method

i $1.37 \times 10^{-10} Q_{W}\left|\omega_{0}\right|$

i $1.77 \times 10^{-10} Q_{W}\left|\mu_{0}\right|^{*}$

i $2.36 \times 10^{-10} Q_{W}\left|\mu_{0}\right|$

i $1.93 \times 10^{-10} \mathrm{OW}_{\mathrm{W}}\left|\mathrm{u}_{0}\right|$

$i(1.51 \pm 0.07) \times 10^{-10} Q_{W}\left|\nu_{0}\right|$

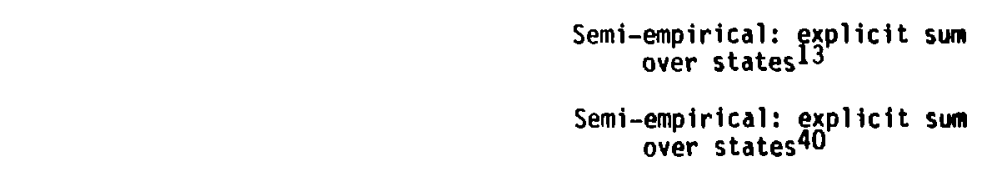

Semi-empirical: explicit sum
over states 13

Semi-empirical: explicit sum Modified
Tietz
Potential $\left[\begin{array}{l}\text { sum } 5 \text { lowest states } 39 \\ \text { Green's function } 39\end{array}\right.$

Relativistic many-body perturbation theory 36

$$
s^{\operatorname{expt} .}=2.8 \pm 0.7^{+0.3}+0.2 \times 10^{-3}
$$

*This result was recalculated to give $~ i 1.64 \times 10^{-10} Q_{W} / \mu_{0} l$ after a sign error in a matrix element was found.

Fig. II-3. Sumary of theoretical predictions for the parity nonconserving $E 1$ amplitude in the $T 1$
$6^{2} \mathrm{P}_{1 / 2} \rightarrow 7^{2} \mathrm{P}_{1 / 2}$ transition. 


$$
\begin{aligned}
& 2 \operatorname{Im}\left(\varepsilon_{\text {PNC }}\right) / M-10^{-3} \\
& 2 \operatorname{Im}\left(\varepsilon_{\text {PMC }}\right) / \varepsilon_{\text {Stark }}-10^{-5} .
\end{aligned}
$$

Neuffer and Commins calculated the Stark amplitudes a and $\mathrm{s}$ were a is the Stark amplitude for the linear polarization of the absorbed radiation parallel to the electric field

$$
\begin{aligned}
& a=\frac{1}{9} \sum_{n S} R_{1 / 2}{ }_{7 P, n S} R_{n S, 6 P}\left(\frac{1}{E_{7 P^{-E} n S}}+\frac{1}{E_{6 P^{-E} n S}}\right) \\
& +\frac{2}{9} \sum_{n D_{3 / 2}} R_{7 P, n S}{ }^{R D, 6 P}\left(\frac{1}{E_{7 P^{-E} n D}}+\frac{1}{E_{6 P^{-E} n D}}\right)
\end{aligned}
$$

and $\beta$ is the Stark amplitude for linear polarization perpendicular to the electric field

$$
\begin{aligned}
& B=\frac{1}{9} \sum_{n S} \sum_{1 / 2} R_{7 P, n S} R_{n S, 6 P}\left(\frac{1}{E_{6 P}-E_{n S}}-\frac{1}{E_{7 P}-E_{n S}}\right) \\
& +\frac{1}{9} \sum_{n D} R_{3 / 2}{ }_{7 P, n S}{ }^{R D, 6 P}\left(\frac{1}{E_{7 P}-E_{n D}}-\frac{1}{E_{6 P}-E_{n D}}\right)
\end{aligned}
$$

where $E_{6 P}=E\left(6^{2} P_{1 / 2}\right)$, etc., and $R_{7 P, n S}=\left\langle 7^{2} P_{1 / 2}|r| n^{2} S_{1 / 2}\right\rangle$, etc. The theoretical values for $\alpha$ and $B$ vary somewhat depending on the calculational method used. One trchnique involves taking a finite sum over the five lowest energy levels using radial integrals calculated by Neuffer and tabulated values for the energy levels. This method yie1ds 39

$$
\begin{aligned}
& e^{2} a=2.43 \times 10^{-5}\left|\frac{e^{h}}{2 m_{e} c}\right| c m / V \\
& e^{2} B=1.78 \times 10^{-5}\left|\frac{e h}{2 m_{e} c}\right| c m / V \\
& B / \alpha=.73 .
\end{aligned}
$$


A Green's function technique was also employed that includes all discrete and continuous $S$ and $D$ states and gives ${ }^{39}$

$$
\begin{aligned}
& e^{2} a=2.05 \times 10^{-5}\left|\frac{e_{h}}{2 m_{e}}\right| \mathrm{cm} / \mathrm{V} \\
& e^{2} B=1.64 \times 10^{-5}\left|\frac{e_{h}}{2 m_{e}}\right| \mathrm{cm} / \mathrm{V} \\
& B / \alpha=.80 .
\end{aligned}
$$

$\alpha$ and $B$ have not been measured directly but the ratio $B / \alpha$ was measured to be $\mathrm{be}^{43}$

$$
(B / \alpha)_{\text {expt. }}=.84 \text {. }
$$

2. Present Work in Thallium

The current thallium experiment at Berkeley employs a method suggested independently by Bouchiat ${ }^{46}$ and Commins ${ }^{47}$ where the $\mathrm{Tl}$ $6^{2} \mathrm{P}_{1 / 2} \rightarrow 7^{2} \mathrm{P}_{1 / 2}$ transition is excited with linearly polarized light passing through $T 1$ vapor in the presence of crossed electric and magnetic fields. If parity is not conserved, then the transition rate contains an interference term between the Stark induced and the PNC electric dipole amplitudes that is proportional to

$$
\hat{\varepsilon} \cdot \vec{B} \cdot \hat{\varepsilon} \cdot \vec{E} \times \vec{B}
$$

where $\hat{E}$ is the laser polarization and $\vec{E}$ and $\vec{B}$ are the electric and magnetic fields respectively.

The coordinate system chosen for the experiment is indicated in fig. Il-4. A laser with $\hat{k}$ parallel to the electric field and tuned to the $6^{2} \mathrm{P}_{1 / 2} \rightarrow 7^{2} \mathrm{P}_{1 / 2}$ transition energy (293 $\mathrm{nm}$ ) excites atoms to the $7^{2} \mathrm{P}_{1 / 2}$ state. In a magnetic field the hyperfine levels are 


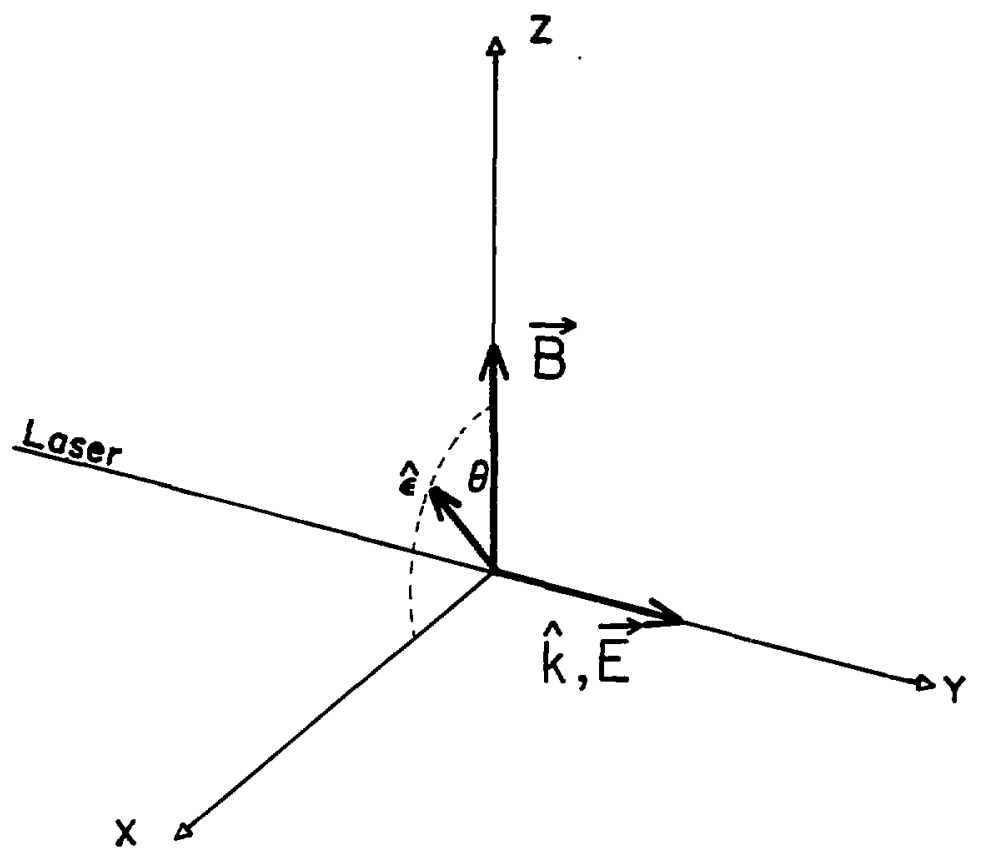

XBL $833-8846$

Fig. II-4. Coordinate system for the experiment giving relative orientations of the laser beam, laser polarization, magnetic and electric fields. 
split as shown in Fig. II-5. Both fsotopes of thallium have a nuclear spin of $1 / 2$ and many of the individual lines $\mid \overline{\left.6^{2} p_{1 / 2} ; F_{,} n_{F}\right\rangle} \rightarrow$ $\left[7^{2} P_{1 / 2} ; F^{\prime} m_{F},\right\rangle$ can be resolved, where $F_{,} F^{\prime}=0,1 ; m_{F}, m_{F^{\prime}}=$ $0, \pm 1$. (The bar over the states indicates the use of states diagonal in the magnetic fieid perturbation.) Whereas the population of either the $\mid 7^{2} \mathrm{P}_{1 / 2} ; \mathrm{F}=0>$ or $17^{2} \mathrm{P}_{1 / 2} ; \mathrm{F}=1>$ state at zero magnetic field does not vary with a parity transformation and no interference between $s$ and epNC exists (see Fig. II-5), the way that the population distributes among individual magnetic sublevels (e.g., $\left|6^{2} \mathrm{P}_{1 / 2} ; \mathrm{F}=0, \mathrm{~m}_{\mathrm{F}}=0\right\rangle \rightarrow\left|7^{2} \mathrm{P}_{1 / 2} ; \mathrm{F}=1, \mathrm{~m}_{\mathrm{F}}=-1\right\rangle$ transition $)$ does exhibit an interference tem. The large magnetic field allows selective excitation of the individual magnetic sublevels and their population is then measured by observing the decay fluorescence as the atoms cascade $7^{2} \mathrm{p}_{1 / 2} \rightarrow 7^{2} \mathrm{~S}_{1 / 2}, 7^{2} \mathrm{~S}_{1 / 2} \rightarrow 6^{2} \mathrm{P}_{3 / 2}, 6^{2} \mathrm{p}_{1 / 2}$ (see fig. II-1). The $7^{2} \mathrm{~S}_{1 / 2} \rightarrow 6^{2} \mathrm{P}_{1 / 2}$ radiation is resonantly trapped at the densities used so the $7^{2} \mathrm{~S}_{1 / 2} \rightarrow 6^{2} \mathrm{P}_{3 / 2}$ transition at $535 \mathrm{~nm}$ is observed. The population of a particular magnetic sublevel varys as the "handedness" of the coordinate system (e.g. the sign of $\hat{\varepsilon} \cdot \vec{B} \hat{\varepsilon} \cdot \vec{E} \times \vec{B}$ ) reverses. The pseudoscalar term in the transition probability, $B \varepsilon_{P N C}$, is extracted by taking the difference in the rate, measured by the number of $535 \mathrm{~nm}$ photons, as the electric field is reversed $\vec{E} \rightarrow-\vec{E}$ and the angle $\theta$ between $\hat{\varepsilon}$ and $\vec{B}$ is reversed $\theta \rightarrow-\theta$. The form of the pseudoscalar provides a signature to help isolate the PNC portion of the transition probability. One looks for a quantity that reverses with $\vec{E}$ and $\theta$ but not with $\hat{k}$ and $\vec{B}$. 


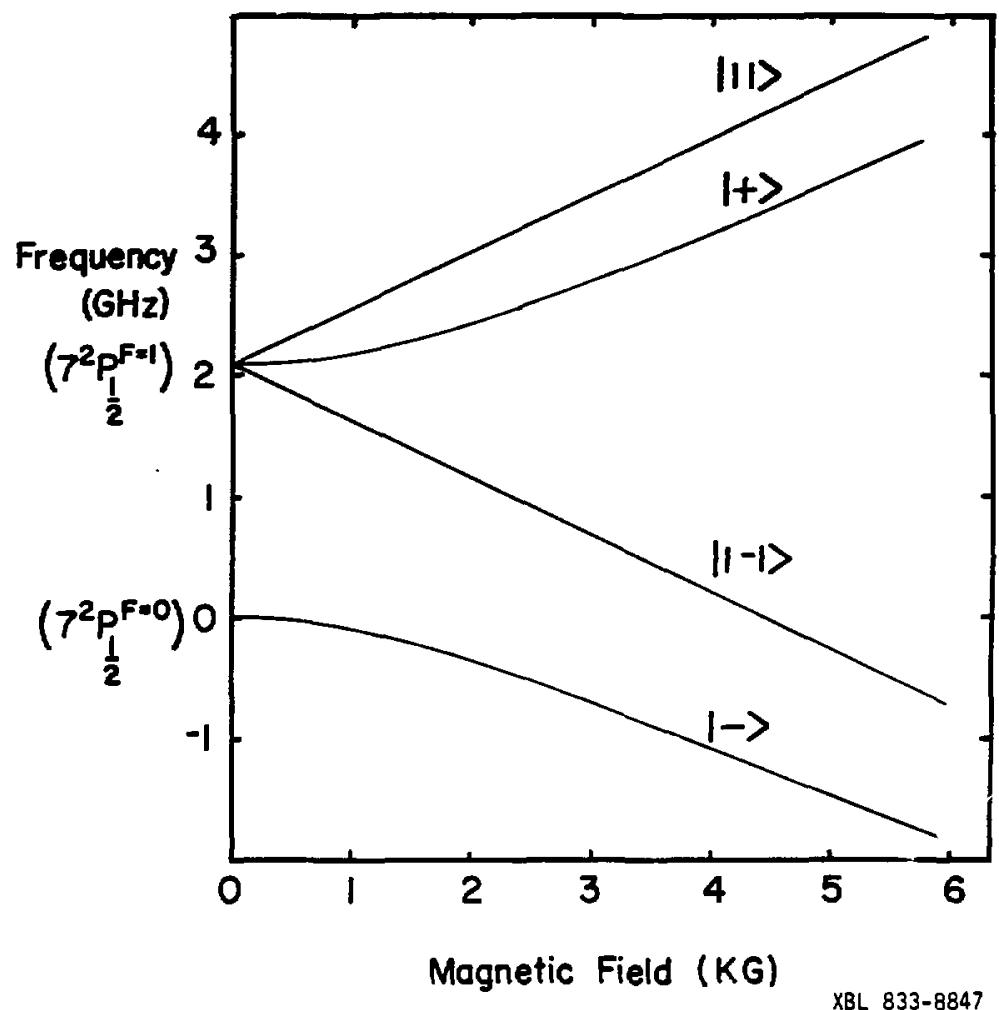

Fig. II-5. Hyperfine splitting of the $7^{2} \mathrm{P}_{1 / 2}$ state of $T 1$ as a function of magnetic field. The zero field hyperfine solitting is $2.1 \mathrm{GHz}$. The eigenstates $|1,1>|+,>$, $|1,-1\rangle$, and $\mid \rightarrow$ are defined in the text in eqns. II.24 and II.25. 
It is straight forward now to proceed and calculate the transition amplitudes between the $6^{2} p_{1 / 2}$ and $7^{2} p_{1 / 2}$ states with the coordinate system defined as in Fig. II-4. Proceeding first with the Stark amplitude, the perturbation Hamiltonian is

$$
\begin{aligned}
H^{\prime} & =\mathrm{eE} \cdot \vec{r} \\
& =\mathrm{eEy} .
\end{aligned}
$$

The perturbed states are

$$
\begin{aligned}
\mid \overline{\left.n^{2} P_{1 / 2}\right\rangle=\left|n^{2} P_{1 / 2}\right\rangle} & +e \sum_{n S} \frac{\left\langle n S|E y| n^{2} P_{1 / 2}\right\rangle}{E_{n P}-E_{n S}}|n S\rangle \\
& +e \sum_{n D} \frac{\left\langle n D|E y| n P_{1 / 2}\right\rangle}{E_{n P}-E_{n D}}|n D\rangle
\end{aligned}
$$

Consider now an electric dipole transition with laser photons

$$
\hat{\varepsilon}=\hat{\varepsilon} \cos \theta+\hat{x} \sin \theta .
$$

The Stark amplitudes are then

$$
\begin{aligned}
& \left.{ }_{\text {Stark }}=e<\overline{7^{2} P_{1 / 2}}|\hat{\varepsilon} \cdot \vec{k}| \overline{6^{2} P_{1 / 2}}\right\rangle \\
& =e^{2} \sum_{n} \frac{\left\langle 7^{2} P_{1 / 2}|z \cos \theta+x \sin \theta| n\right\rangle\left\langle n|E y| 6^{2} P_{1 / 2}\right\rangle}{E_{6 P}-E_{n}} \\
& +e^{2} \sum_{n} \frac{\left\langle 7^{2} p_{1 / 2}|E y| n>\left\langle n|z \cos \theta+x \sin \theta| 6^{2} p_{1 / 2}\right\rangle\right.}{E_{7 p}-E_{n}}
\end{aligned}
$$

where $\mathrm{n}=\mathrm{nS}_{1 / 2}, \mathrm{nD}_{3 / 2}$ states. Standard computations lead to the $2 \times 2$ transition matrix:

$$
\varepsilon_{\text {Stark }}=e^{2} E^{\star}
$$




$$
\begin{array}{ll|lll} 
& & \frac{1}{2} & -\frac{1}{2} & m_{j}\left(6^{2} \mathrm{p}_{1 / 2}\right) \\
\cline { 2 - 3 } m_{j}\left(7^{2} P_{1 / 2}\right) & \frac{1}{2} & i \beta \sin \theta & -i \beta \cos \theta \\
& -\frac{1}{2} & -i \beta \cos \theta & -i \beta \sin \theta
\end{array}
$$

with $B$ defined in eqn. II.9.

The MI matrix elements are equally straight forward to generate in terms of the calculated value

$$
M=\left\langle 7^{2} p_{1 / 2} ; m_{j}=+1 / 2\left|M 1_{o p}\right| 6^{2} p_{1 / 2} ; m_{j}=+1 / 2\right\rangle \text {. }
$$

The angular part of the $M I$ operator is described by the familiar

$$
\vec{\mu} \cdot \hat{k} \times \hat{\mathbf{E}}
$$

which gives

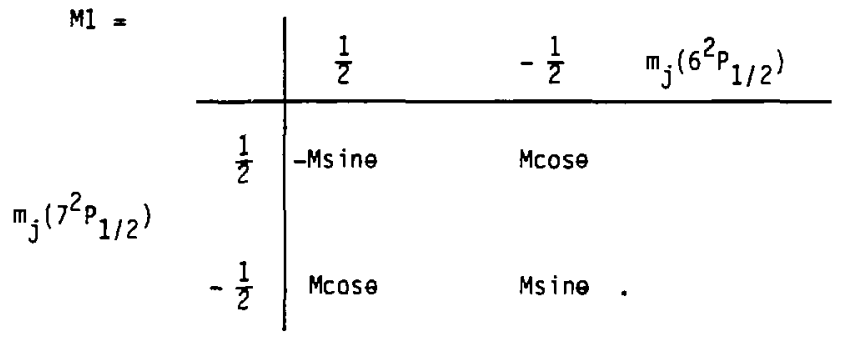

Finally, using II.3, the definition of EPNC (II.4) and the substitution

$$
U=E_{\text {PNC }} / i
$$

the parity nonconserving amplitudes are

$$
\begin{aligned}
& \varepsilon_{\text {PNC }}= \\
& \begin{array}{cc|ccc} 
& & \frac{1}{2} & \frac{1}{2} & m_{j}\left(6^{2} p_{1 / 2}\right) \\
\cline { 2 - 4 } m_{j}\left(7^{2} p_{1 / 2}\right) & \frac{1}{2} & -i U \cos \theta & -i U \sin \theta \\
& -\frac{1}{2} & -i U \sin \theta & \text { iUcos }
\end{array}
\end{aligned}
$$


The hyperfine splittings are large so the most convenient basis states are those described by $\left|F, m_{F}\right\rangle$

$$
\begin{aligned}
& \left|F, m_{F}\right\rangle \quad\left|m_{j}, m_{i}\right\rangle \\
& |0,0\rangle=(|1 / 2,-1 / 2\rangle-|-1 / 2,1 / 2\rangle) / \sqrt{2} \\
& |1,1\rangle=|1 / 2,1 / 2\rangle \\
& |1,0\rangle=(\mid 1 / 2,-1 / 2)+|-1 / 2,1 / 2\rangle) / \sqrt{2} \\
& |1,-1\rangle=|-1 / 2,-1 / 2\rangle .
\end{aligned}
$$

The total transition matrix on this basis is given in Fig. II-6. As was mentioned before, none of the four possible transitions $F=0 \rightarrow F^{\prime} \quad 0, F=0 \rightarrow F^{\prime}=1, F=1 \rightarrow F^{\prime}=0, F=1 \rightarrow F^{\prime}=1$ has a $\mathrm{BU}$ interference term. In order to see an interference a magnetic field strong enough to resolve the hyperfine lines must be applied. In the intermediate field Zeeman effect (where ${ }_{\mu} B$ hyperfine splitting) for a magnetic field

$$
\vec{B}=B \hat{z},
$$

the eigenstates of the perturbation are

$$
\begin{array}{ll}
\mid 1,1> & E_{11}=\Delta(1+x / 2) \\
|+>=\delta| 10>+\gamma \mid 00> & \dot{E}_{+}=\Delta\left(1+\sqrt{1+x^{2}}\right) / 2 \\
\mid 1,-1> & E_{1-1}=\Delta(1-x / 2) \\
|->=\delta| 10>-Y \mid 00> & E_{-}=\Delta\left(1-\sqrt{1+x^{2}}\right) / 2
\end{array}
$$

where the energy levels are shifted as indicated and: 


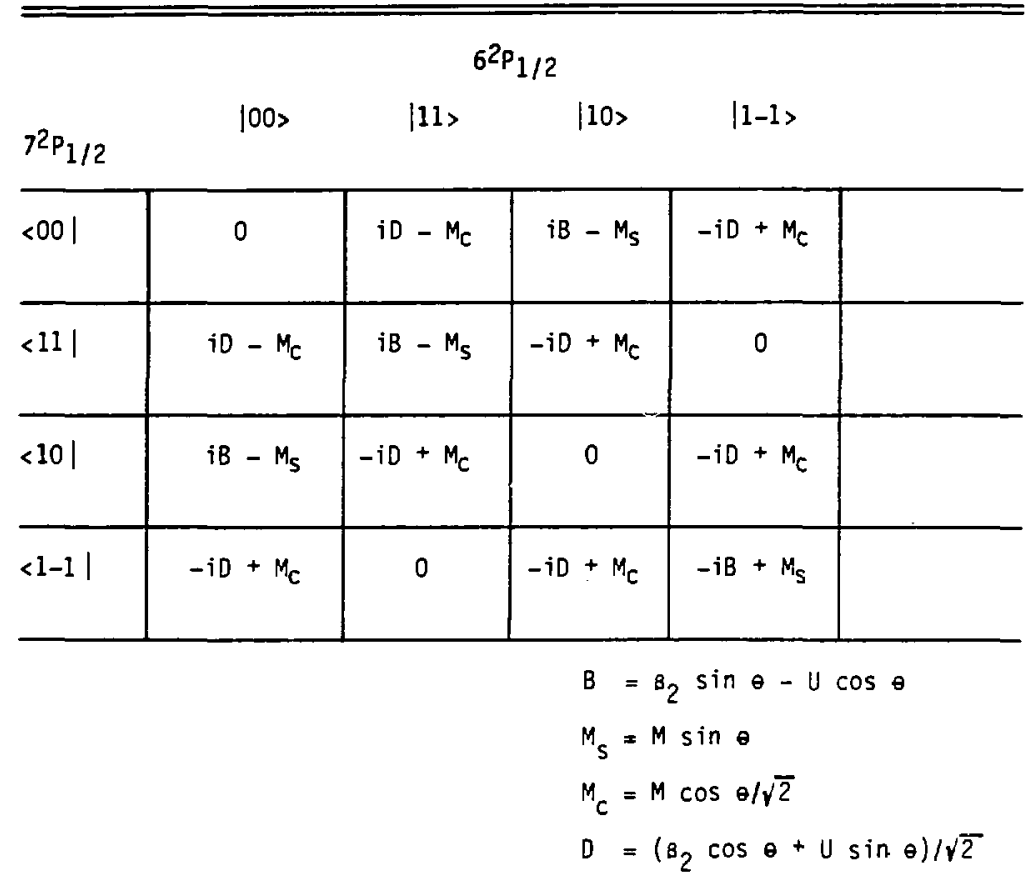

Fig. II-6. Dipole transition amplitudes $\langle M l\rangle+\left\langle E_{P N C}\right\rangle+\left\langle E l_{\text {Stark }}\right\rangle$ for the $6^{2} P_{1 / 2}\left|F, m_{F}>\rightarrow 7^{2} P_{1 / 2}\right| F^{\prime}$, mF $1>$ transitions. 


$$
\begin{aligned}
& { }^{u_{0}}=\text { Bohr magneton }=1.4 \mathrm{MHz} / \text { gauss } \\
& \Delta=\text { hyperfine splitting of } F=0 \text { and } F=1
\end{aligned}
$$

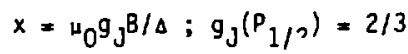

$$
\begin{aligned}
& \delta=\frac{x}{\sqrt{x^{2}+\left(1-\sqrt{1+x^{2}}\right)^{2}}} \\
& Y=\frac{\sqrt{1+x^{2}}-1}{\sqrt{x^{2}+\left(1-\sqrt{1+x^{2}}\right)^{2}}} .
\end{aligned}
$$

Figure II-7 lists the transition probabilities and energy shifts of these new eigenstates with the coefficient of $28 \mathrm{U}$, the Stark-PNC interference term, explicitly listed. Terms of order $M^{2}$ or $U^{2}$ are not included. Numerical valves for the coefficients with $B=4$ KGauss are given in addition.

The transition most attractive to work on is $\left|6^{2} p_{1 / 2} ; 1,1\right\rangle \rightarrow$ $\left|7^{2} \mathrm{p}_{1 / \mathrm{c}} ; 1,1\right\rangle$ which coincides with $\left|6^{2} \mathrm{p}_{1 / 2} ; 1,-1\right\rangle \rightarrow$ $\mid 7^{2} \mathrm{P}_{1 / 2} ; 1,-1>$ and is therefore the strongest. The transition probability, $T^{\theta}$, is

$$
\begin{aligned}
T_{11 \rightarrow 11}^{\theta}-E^{2} B^{2} \sin ^{2} \theta & -2 E_{B} U \sin \theta \cos \theta+U^{2} \cos ^{2} \theta \\
& +M^{2} \sin ^{2} \theta \\
\sim & E^{2} B^{2} \sin ^{2} \theta-2 E B U \sin \theta \cos \theta .
\end{aligned}
$$

The intensity asymmetry for $+_{\theta} \rightarrow-\theta$ (or likewise $+E \rightarrow-E$ ) is

$$
\begin{aligned}
\Delta_{11 \rightarrow 11}^{\theta} & =\frac{T_{11 \rightarrow 11^{(+)}-T_{11 \rightarrow 11}(-)}}{T_{11 \rightarrow 11^{(+)}+T_{11 \rightarrow 11^{(-)}}}} \\
& =-\frac{2 ! 1}{E B} \cot \theta .
\end{aligned}
$$

To choose precisely the cor act magnetic fielo and valve of $\theta$ for which the experiment is optimized requires a computer calculation. Doppler broadened nearby lines with $\Delta$ 's of the opposite sign can 
Transition

$4 K G$

${ }_{B_{2}}{ }^{2} \sin ^{2} \theta \quad{ }^{2} B_{2} U \sin \theta \cos \theta \quad$ Freq. (GHz) Amp. ${ }^{B_{B}}{ }^{2}$

\begin{tabular}{lcccc}
\hline$T(11 \rightarrow 11)$ & 1 & -1 & 0 & $\sin ^{2} \theta$ \\
$T(1-1 \rightarrow 1-1)$ & 1 & -1 & 0 & $\sin ^{2} \theta$ \\
$T(+\rightarrow+)$ & $\omega^{2}$ & $-\omega^{2}$ & .91 & $.33 \sin ^{2} \theta$ \\
$T(\rightarrow-)$ & $\omega^{2}$ & $-\omega^{2}$ & 18.29 & $.33 \sin ^{2} \theta$ \\
$T(+\rightarrow-)$ & $\phi^{2}$ & $-\omega^{2}$ & -3.36 & $.67 \sin ^{2} \theta$ \\
$T(\rightarrow+)$ & $\phi^{2}$ & $-\omega^{2}$ & 22.56 & $.67 \sin ^{2} \theta$ \\
\hline
\end{tabular}

Fig. II $-7(a)$

Transition probabilities for $6^{2} \mathrm{p} 1 / 2 \rightarrow 7^{2} \mathrm{p} 1 / 2$ transitions in a magnetic field, for (a) $\Delta m_{F}=0$ ani $(b) \Delta m_{F}= \pm 1$. The first column gives the transition strength, column 2 gives the sign and coefficient of the PNC interference term, and columns 3 and 4 give numerical values for energy splittings and Tine strengths for $B=4$ KGauss. The symbols used are defined as follows (refer to II.26 in text):

$$
\begin{gathered}
\omega=\delta_{7} \gamma_{6}+\delta_{6} \gamma_{7} \\
\sigma=\delta_{7} \delta_{6}-{ }_{7} \gamma_{6} \\
\Lambda_{6(7)}=\delta_{6(7)}+\gamma_{6(7)} \\
\Gamma_{6(7)}=\delta_{6(7)}-\gamma_{6(7)}
\end{gathered}
$$


Transition

4 KG

$\theta_{2}{ }^{2} \cos ^{2} \theta \quad{ }^{2 B_{2}}$ Usinecose Freq. (GHz) Amp. $\star_{B_{2}}{ }^{2}$

\begin{tabular}{lllll}
\hline$T(11 \rightarrow-)$ & $\frac{1}{2} \Lambda_{7}^{2}$ & $\frac{1}{2} \Lambda_{7}^{2}$ & -5.08 & $.94 \cos ^{2} \theta$ \\
$T(1-1 \rightarrow+)$ & $\frac{1}{2} \Lambda_{7}^{2}$ & $\frac{1}{2} \Lambda_{7}^{2}$ & 2.96 & $.94 \cos ^{2} \theta$ \\
$T(t \rightarrow 1-1)$ & $\frac{1}{2} \Lambda_{6}^{2}$ & $\frac{1}{2} \Lambda_{6}^{2}$ & -2.04 & $.59 \cos ^{2} \theta$ \\
$T(\rightarrow 11)$ & $\frac{1}{2} \Lambda_{6}^{2}$ & $\frac{1}{2} \Lambda_{6}^{2}$ & 23.36 & $.59 \cos ^{2} \theta$ \\
$T(11 \rightarrow+)$ & $\frac{1}{2} \Gamma_{7}^{2}$ & $\frac{1}{2} \Gamma_{7}^{2}$ & -.81 & $.06 \cos ^{2} \theta$ \\
$T(1-1 \rightarrow-)$ & $\frac{1}{2} r_{7}^{2}$ & $\frac{1}{2} \Gamma_{7}^{2}$ & -1.32 & $.06 \cos ^{2} \theta$ \\
$T(\rightarrow 1-1)$ & $\frac{1}{2} \Gamma_{6}^{2}$ & $\frac{1}{2} r_{6}^{2}$ & 19.60 & $.41 \cos ^{2} \theta$ \\
$T(t \rightarrow 11)$ & $\frac{1}{2}{ }_{6}^{2}$ & $\frac{1}{2} r_{6}^{2}$ & 1.72 & $.41 \cos ^{2} \theta$ \\
\hline \hline
\end{tabular}

Fig. $I I-7(b)$ 
dilute the observed asymmetry. Also, $\Delta$ does not approach infinity as - goes to zero as might be indicated by 11.28 since the signal $\left(-\sin ^{2} a\right)$ at that point slips below the background and instead $\Delta \rightarrow$ 0. In order to minimize the running time for the experiment, the quantity

$$
K=\frac{1}{\left(\Delta^{\circ}\right)^{2} T^{\theta}}
$$

must be minimized. This follows if the experiment is limited by counting statistics, since then the uncertainty in the measured as ymmetry is $1 / \sqrt{N}$ where $N$ is the number of $535 \mathrm{~nm}$ photons counted:

$$
N=k^{\prime} T^{\theta} t
$$

where $k^{\prime}$ is a constant and $t$ is the running time. In II.29 the effects due to nearby lines and finite background must be included in $\Delta^{\theta}$ and $T^{\theta}$. The necessary computer calculations have been done and a detailed description along with actual laser scans of the hyperfine structure will be presented in Chapter III and IV.

The analysis presented in this chapter is correct as far as it goes, but is inadequate to describe an actual experiment. In the real experiment, the electric and magnetic fields are not precisely perpendicular to $k$, it is not possible to make $E \rightarrow-E$ or $\theta \rightarrow-\theta$ exactly, the linear polarization is actually slightly elliptical, and the background is not a constant. Ail of these misalignments and imperfections can cause false asymmetries, many of which have signatures disturbingly like that of parity nonconservation. The 
challenge of this experinent is to separate the false effects from the real PHC. The effect one wishes to measure is very small and the elimination of the impostors is quite tricky. The next chapter will be devoted to the analysis of possible false effects and how to remove them. 


\section{AMALYSIS OF SYSTEMATICS}

\section{Design Considerations}

This experiment looks for a small intensity asymetry $\left(-10^{-5}\right)$ that violates parity. A reliable measurement requires that any systematic asymmetry inherent in the instrument with the same signature as PAC be removed to the $10^{-6}$ level. The design of the experiment, with a maximal amount of symmetry built in, avoids a great deal of possible instrumental asymmetry. Two interaction regions side by side in the thallium vapor cell, viewed independently, which share the same laser pulse and $T 1$ density but in which the electric fields are opposite, allow an asymmetry to be measured on a shot-to-shot basis. This removes possible effects due to fluctuations in the laser power or in the density. A mirror (see Fig. IV-5) at the end of the cell can reflect the laser light back through the cell, thus removing possible effects that depend on $\hat{k}$. A further effort is made to reduce systematic drifts with time by changing as many parameters of the experiment on as short a time scale as possible. The electric field and the laser polarization change on a shot-to-shot basis at $16 \mathrm{~Hz}$ (the rep. rate of the laser). The frequency of the laser changes at 1 $\mathrm{Hz}$, and data are taken on transitions of opposite asymmetry. The mirror which reverses $\hat{k}$ is blocked and unblocked once per minute and $\vec{B}$ is reversed every $15 \mathrm{~min}$. Nevertheless, these efforts are not sufficient to guarantee that the systematics are at the $10^{-6}$ level or below. Misalignments, backgrounds and imperfect subtractions can all contribute to mask the PNC asymmetry. What follows is a complete analys is of the possible false effects that can occur, and ways that they can be distinguished from true parity nonconservation. 


\section{Misalignments and Imperfect Subtractions}

The transition amplitudes given in Fig. II-6 describe transitions for an atom in an electric field exactly along $\hat{y}$ with perfectly linearly polarized incident laser light. Figure $\mathrm{II}-7$ lists the transition probabilities for the atom when a magnetic field exactly along $\hat{z}$ is applied. Let the laser beam, $\hat{k}$, define the $y$ axis. Suppose then that $\vec{E}$ is not parallel to $\hat{k}$ and $\vec{B}$ is not perpendicular to $\hat{k}$ as shown in Fig. III-I, and that the light is not perfectly polarized. Figure II-6 and II-7 must then be expanded to include terms in the amplitude that these misalignments will introduce.

First consider the result of having the electric field not be parallel to $\hat{k}$ with zero magnetic field. Then,

$$
\begin{aligned}
& \vec{E}=E_{x} \hat{x}+E_{y} \hat{y}+E_{z} \hat{z} \\
& E_{x}, E_{z} \ll E_{y}
\end{aligned}
$$

and the perturbed states (II.15) are changes by the replacement

$$
E_{y} y \rightarrow E_{x} x+E_{y} y+E_{z} z
$$

II.18 is now modified by the addition of terms in the amplitude proportional to $E_{x}$ and $E_{z}$. Using the following shorthand

$$
\begin{array}{ll}
a_{1}=a E_{x} & a_{1}=a E_{x} \\
a_{2}=a E_{y} & B_{2}=\theta E_{y} \\
a_{3}=a E_{z} & B_{3}=\mathrm{aE}_{z}
\end{array}
$$

with $a$ and $B$ as defined in II.8 and II.9, the Stark transition matrix II .18 beccmes 


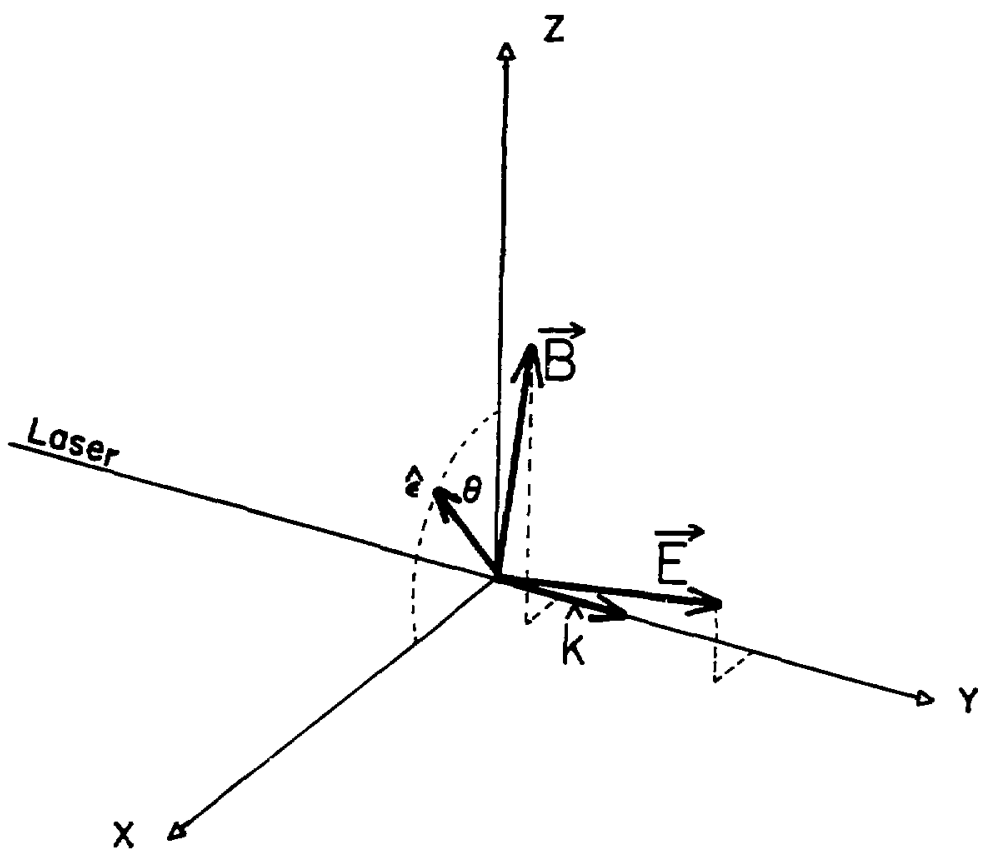

XBL $833-8848$

Fig. III-1. Schematic view of the actual coordinate system for the experiment where $E$ and $B$ are not exactly aligned with the $y$ and $z$ axes respectively (exaggerated view). 


$$
\begin{aligned}
& \varepsilon_{\text {Stark }}=\mathrm{e}^{2}
\end{aligned}
$$

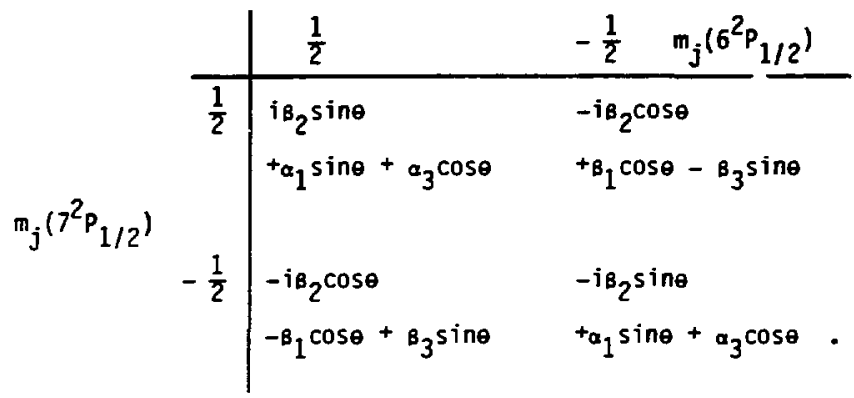

It is also possible and indeed certain that the nominally linearly polarized light is in fact elliptically polarized. Arbitrary polarization in the $x, z$ plane ( $k$ defines $y$ ) can be written

$$
\hat{\varepsilon}=\hat{z} \cos \theta+\dot{x} e^{i \delta} \sin \theta .
$$

At this point there is nothing in the experiment that defines the $z$ axis $(B=0$ here). Define the $x$ axis (thereby determining the $z$ axis as $\hat{z}=\hat{x} \times \hat{y}$ ) to be the axis that bisects the angle between $\theta^{+}$and $\theta-$ a priori. The magnetic field, which is nominally along $z$, does not exactiy bisect the angle between the two choices for linear polarization. Defining $x$ as the axis that the two signs of linear polarization are symmetric about is equivalent then to admitting an $x$-component to the magnetic field (see Fig. III-1). The amount of birefringence in the system, $\sigma_{\varepsilon}$, is measured to be very small so III.4 can be expanded assuming $\delta_{\varepsilon} \ll 1$ to give:

$$
\hat{\varepsilon}=\cos \theta \hat{z}+\left(1+i \delta_{\varepsilon}\right) \sin \theta \hat{x} .
$$

The correct form of Fig. II-6, where possible misalignments in $E$ and imperfections in the linear polarizatin are taken into account is 
given in Fig. III-2. It is already clear that $E_{x}, E_{z}$ and $\delta_{c}$ are going to cause trouble - give rise to terms in the transition probability that look similar to PNC. As an example, consider a magnetic field applied exactly along the z-axis. (The possibility of off axis magnetic field components will be dealt with presently.) From the definitions (II.25) and the observed symmetry in Fig. III-2, one can write

$$
\begin{aligned}
A_{+\rightarrow_{+}}=\rho A_{10 \rightarrow 10}+\omega_{10} & \rightarrow 00 \\
\rho & =\delta_{7} \delta_{6}+\gamma_{7} \gamma_{6} \\
\omega & =\delta_{7} \gamma_{6}+\gamma_{7} \delta_{6}
\end{aligned}
$$

where $A_{a \rightarrow b}$ refers to the transition amplitude from state las to state $|b\rangle$. Fig. III-2 gives

$$
\begin{aligned}
& A_{10 \rightarrow 10}=\left(1+i \delta_{\varepsilon}\right) a_{1} \sin \theta+a_{3} \cos \theta \\
& A_{10 \rightarrow 00}=i\left(1+i \delta_{\varepsilon}\right) \theta_{2} \sin \theta-i U \cos \theta-M \sin \theta\left(1+i \delta_{\varepsilon}\right) .
\end{aligned}
$$

Squaring to get the transition probability $T$ and keeping terms to second order only in the small quantities $a_{1}, a_{3}, \delta_{\varepsilon}, M_{s}$ and to first order only in $U$ gives

$$
\begin{aligned}
T_{+\rightarrow^{+}} & =\left(\omega^{2} \theta_{2}^{2}+\omega^{2} \delta_{c}^{2} \beta_{2}^{2}+\omega^{2} M^{2}+\rho^{2} a_{1}^{2}\right) \sin ^{2} \theta+\rho^{2} a_{3}^{2} \cos ^{2} \theta \\
& +2\left(\rho^{2} a_{1} a_{3}-\omega^{2} \beta_{2} U-\rho \omega a_{3}-\rho \omega \delta_{\varepsilon} \theta_{2} a_{3}\right) \sin \theta \cos \theta .
\end{aligned}
$$

Clearly,

$$
\omega^{2} \beta_{2}^{2} \gg \omega^{2} \delta_{\varepsilon}^{2} \beta_{2}^{2}, \omega^{2} M^{2}, \rho^{2} a_{1}^{2}, \rho^{2} a_{3}^{2} \cot ^{2} \theta
$$

so the expression reduces to

$$
T_{+_{+^{+}}}=\omega^{2} \theta_{2}^{2} \sin ^{2} \theta+2\left(\rho^{2} a_{1} a_{3}-\omega^{2} \beta_{2} U-\nu \omega M a_{3}-\nu \omega \delta_{\varepsilon} \beta_{2} a_{3}\right) \sin \theta \cos \theta .
$$




\section{${ }^{2} \mathrm{p}_{1 / 2}$}

$\begin{array}{lllll}7^{2} p_{1 / 2} & |00\rangle & |11\rangle & |10\rangle & |1-1\rangle\end{array}$

\begin{tabular}{c|c|c|c|c}
\hline$<00 \mid$ & $A$ & $C+i D-M_{C}$ & $i B-M_{S}$ & $C-i D+M_{C}$ \\
\hline$<11 \mid$ & $-C+i D-M_{C}$ & $A+i B-M_{S}$ & $C-i D+M_{C}$ & 0 \\
\hline$<10 \mid$ & $i B-M_{S}$ & $-C-i D+M_{C}$ & $A$ & $C-i D+M_{C}$ \\
\hline$<1-1 \mid$ & $-C-i D+M_{C}$ & 0 & $-C-i D+M_{C}$ & $A-i B+M_{S}$ \\
\hline
\end{tabular}

$$
\begin{aligned}
& A=\left(1+i \delta_{\varepsilon}\right) a_{1} \sin \theta+a_{3} \cos \theta \\
& B=\left(1+i \delta_{\varepsilon}\right) B_{2} \sin \theta-U \cos \theta \\
& M_{S}=\left(1+i \delta_{\varepsilon}\right) M \sin \theta \\
& M_{C}=M \cos \theta / \sqrt{2} \\
& C=\left(B_{1} \cos \theta-\left(1+i \delta_{\varepsilon}\right) B_{3} \sin \theta\right) / \sqrt{2} \\
& D=\left(B_{2} \cos \theta+U \sin \theta\right) / \sqrt{2}
\end{aligned}
$$

Fig. III-?: Dipole transition amplitudes 〈MI $\rangle+\left\langle E_{\text {PNC }}\right\rangle+\left\langle E l_{\text {Stark }}\right\rangle$ for the $6^{2} P_{1 / 2}\left|F, m_{F}>\rightarrow 7^{2} P_{1 / 2}\right| F F^{\prime}, m_{F} />$ transitions with misaligned electric field and imperfect linear polarization. 
The term of interest is $-2 \omega^{2} \theta_{2}$ Usinecose, the PNC interference. Yet, it is clear that on this transition the term $-2 p$ wha ${ }_{3} \sin \theta \cos \theta$, an interference between the magnetic dipole and the Stark assisted electric dipole due to an off axis component of $\vec{E}$, behaves the same way as the parity term does under reversal of $\vec{E}$ and $\theta$. Furthermore, consider the terms $\left(20^{2} \alpha_{1} a_{3}-20 \omega \delta_{1}{ }_{2} a_{3}\right) \sin \theta \cos \theta$. These have the same $\theta$ dependence as the parity term but are quadratric rather than linear in $E$. These terms should be el iminated for the most part by the subtraction $E^{+} \rightarrow E-$, but the electric field does not reverse exactly so the subtraction is not perfect. The electric field in the interaction region has a part that reverses $E^{+} \rightarrow E-$ as well as small nonreversing components. Let $E_{0 x}, E_{o y}$, and $E_{O z}$ be the reversing components of the field while $\Delta E_{x}, \Delta E_{y}$, and $\Delta E_{z}$ are the nonreversing components.

$$
\vec{E}=\left(E_{o x}+\Delta E_{x}\right) \hat{x}+\left(E_{o y}+\Delta E_{y}\right) \hat{y}+\left(E_{o z}+\Delta E_{z}\right) \hat{z} \text {. }
$$

With (III.10) and (III.2) the terms quadratic in $E$ become

$$
\begin{aligned}
& 2\left(\rho^{2} a_{1} a_{3}-o \omega \delta_{\varepsilon}{ }^{\theta_{2} a_{3}}\right) \sin \theta \cos \theta=2\left[\rho^{2} \alpha^{2}\left(E_{0 x} E_{0 z}+\Delta E_{x} \Delta E_{2}\right)\right. \\
& \text { - } \left.\rho \omega \delta_{c} a \beta\left(E_{o y} E_{0 z}+\Delta E_{y} \Delta E_{z}\right)\right] \sin \theta \cos \theta \\
& +2\left[\rho^{2} \alpha^{2}\left(E_{o x} \Delta E_{z}+E_{0 z} \Delta E_{x}\right)\right. \\
& \text { - } \left.\rho \omega \sigma_{\varepsilon} a \beta\left(E_{o y} \Delta E_{z}+E_{o z} \Delta E_{y}\right)\right] \sin \theta \cos \theta \text {. }
\end{aligned}
$$

The first term in brackets does not reverse sign when the subtraction $E^{+} \rightarrow E-$ is made, but the second term does change sign and therefore mimics the PNC term under both rever'sal of $E$ and of $\theta$. The 
misalignent combines with an imperfect subtraction to give a false asyminetry. 48

There are subtractions that remain to differentiate between the 3 false terms in (III.9) and the ${ }_{2} U$ term. How these false asymmetries can be differentiated from the PNC asymmetry will be discussed later in this chapter and primarily in Chapter V. First, the effects due to a misaligned magnetic field must be considered.

The actual magnetic field in the experiment can be written

$$
\vec{B}=8_{x} \hat{x}+8_{y} \hat{y}+8_{z} \hat{z}
$$

with

$$
B_{z} \gg B_{x}, B_{y} \cdot
$$

$B_{x}$ and $B_{y}$ are treated as a perturbation, using as a basis the states defined in (II.25). The transition amplitudes between these states perturbed by the off ax is components of $B$ can be written in. terms of the states unperturbed by the magnetic field so that the amplitudes can then be read off in a straight forward way. They are given in Fig. III-3. Using Fig. III-2 with Fig. III-3, the total transition probabilities can be written where now all possible misalignments are included. Terms even in $\theta$ or even in E (e.g. $E_{0} E_{0}$ or $\triangle E \Delta E$ terms) are of no consequence except for the dominant $B_{2}^{2}$ term that gives the strength of the line. Terms odd in $E$ and in $\theta$ are of great consequence however, and a tabulation of the problematical terms is given in Fig. III-4.

For many of the systematics, Fig. III-4 provides information on how to eliminate them. For instance, working on the $|1,1\rangle \rightarrow|1,1\rangle$, $|1,-1\rangle \rightarrow|1,-1\rangle$ line eliminates the $a_{3} M$ and $\delta_{\varepsilon_{2}} 2^{a_{3}}$ terms since 
$\Delta m_{F}=0$ transitions:

$$
\begin{aligned}
& \bar{A}_{11 \rightarrow 11}=A_{11 \rightarrow 11}+\frac{1}{\sqrt{2}} \theta_{-} A_{11 \rightarrow 10}+\frac{1}{\sqrt{2}} \theta_{+} A_{10 \rightarrow 11} \\
& \bar{A}_{1-1 \rightarrow 1-1}=A_{1 \rightarrow 1 \rightarrow 1-1}-\frac{1}{\sqrt{2}} \theta_{-} A_{11 \rightarrow 10}-\frac{1}{\sqrt{2}} \theta_{+} A_{10 \rightarrow 11} \\
& \bar{A}_{++}=\rho A_{10 \rightarrow 10}+\omega A_{10 \rightarrow 00}+\omega\left(\theta_{-} A_{11 \rightarrow 10}+\theta_{+} A_{10 \rightarrow 11}\right) / \sqrt{2} \\
& \bar{A}_{\rightarrow-}=A_{10 \rightarrow 10}-\omega A_{10 \rightarrow 00}-\omega\left(\theta_{-} A_{11 \rightarrow 10}+\theta_{+} A_{10 \rightarrow 11}\right) / \sqrt{2} \\
& \bar{A}_{+\rightarrow}=A_{10 \rightarrow 10}+A_{10 \rightarrow 00}+\omega\left(\theta_{-} A_{11 \rightarrow 10}+\theta_{+} A_{10 \rightarrow 11}\right) / \sqrt{2} \\
& \bar{A}_{\rightarrow_{+}}=-\infty A_{10 \rightarrow 10}+\omega A_{10 \rightarrow 00}+\omega\left(\theta_{-} A_{11 \rightarrow 10}+\theta_{+} A_{10 \rightarrow 11}\right) / \sqrt{2}
\end{aligned}
$$

$\Delta m_{F}= \pm 1$ transitions:

$$
\begin{aligned}
& \bar{A}_{11 \rightarrow-}=-\Lambda_{7} A_{11 \rightarrow 10}+\Lambda_{7} \theta_{+} A_{10 \rightarrow 00} / \sqrt{2} \\
& \bar{A}_{1-1 \rightarrow+}=\Lambda_{7} A_{10 \rightarrow 11}-\Lambda_{7} \theta_{-} A_{10 \rightarrow 00} / \sqrt{2} \\
& \bar{A}_{+\rightarrow 1-1}=\Lambda_{6} A_{11 \rightarrow 10}-\Lambda_{6} \theta_{+} A_{10 \rightarrow 00} / \sqrt{2} \\
& \bar{A}_{\rightarrow 11}=-\Lambda_{6} A_{10 \rightarrow 11}+\Lambda_{6} \theta_{-} A_{10 \rightarrow 00} / \sqrt{2} \\
& \bar{A}_{11 \rightarrow+}=\Gamma_{7} A_{11 \rightarrow 10}-\Gamma_{7} \theta_{+} A_{10 \rightarrow 00} / \sqrt{2} \\
& \vec{A}_{1-1 \rightarrow-}=\Gamma_{7} A_{10 \rightarrow 11}-\Gamma_{7} \theta_{-} A_{10 \rightarrow 00} / \sqrt{2} \\
& \bar{A}_{+\rightarrow 11}=\Gamma_{6} A_{10 \rightarrow 11}-\Gamma_{6} \theta_{-} A_{10 \rightarrow 00} / \sqrt{2} \\
& \bar{A}_{\rightarrow 1-1}=\Gamma_{6} A_{11 \rightarrow 10}-\Gamma_{6} \theta_{+} A_{10 \rightarrow 00} / \sqrt{2}
\end{aligned}
$$$$
\theta_{ \pm} \pm \frac{B_{x} \pm i B_{y}}{2}
$$

$$
\begin{aligned}
& \rho=\delta \delta^{\delta} \delta_{6}+\gamma_{7} \gamma_{6} \\
& \alpha=\delta \gamma_{6}-\gamma_{7} \delta_{6} \\
& \omega=\delta_{7} \gamma_{6}+\gamma_{7} \delta_{6} \\
& \phi=\delta \delta^{\delta} \delta_{6}-\gamma_{7} \gamma_{6} \\
& \Gamma=\delta-\gamma \\
& \Lambda=\delta+\gamma
\end{aligned}
$$

Fig. III-3. Transition amplitudes for $6^{2} \mathrm{P}_{1 / 2}\left|\mathrm{~F}, \mathrm{mF}_{\mathrm{F}} \mathrm{l}\right\rangle \rightarrow\left|7^{2} \mathrm{p}_{1 / 2} \mathrm{~F}^{\prime}, \mathrm{mF}_{\mathrm{F}}\right\rangle$ transitions for the atom in a magnetic field primarily along $z$ with small $x$ and $y$ components. The amplitudes are written in terms of the 0 magnetic field transition amplitudes. 


\begin{tabular}{|c|c|c|c|c|c|c|c|c|}
\hline & \multirow[b]{2}{*}{$\mathrm{B}_{2}{ }^{2}$} & \multirow[b]{2}{*}{$\sin ^{2} \theta$} & \multicolumn{6}{|c|}{ Interference Terms $* \sin \theta \cos \theta$} \\
\hline & & & ${ }^{2 B_{2} U}$ & ${ }^{2 a_{1}} a_{3}$ & $2 a_{3} M$ & ${ }^{2 B} 1_{1}{ }_{2}{ }^{\theta} y$ & $2 \beta_{2}^{2}{ }^{\theta} x$ & $2 \delta_{E}^{\beta} 2^{\alpha_{3}}$ \\
\hline$T(11 \rightarrow 11)$ & & 1 & -1 & +1 & -1 & +1 & -1 & -1 \\
\hline $\mathrm{T}(1-1 \rightarrow 1-1)$ & & 1 & -1 & +1 & +1 & +1 & -1 & +1 \\
\hline$T(+>+)$ & & $\omega^{2}$ & $-\omega^{2}$ & $+0^{2}$ & ש L & $+\omega^{2}$ & $-\omega^{2}$ & - Dw \\
\hline$T(\rightarrow-)$ & & $\omega^{2}$ & $-\omega^{2}$ & $t_{p}^{2}$ & $t_{\infty}$ & $+\omega^{2}$ & $-\omega^{2}$ & $+\infty \omega$ \\
\hline$T(+\rightarrow)$ & & $\psi^{2}$ & $-\phi^{2}$ & $+\phi^{2}$ & $-\phi \psi$ & $+\psi^{2}$ & & 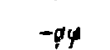 \\
\hline$T(\rightarrow+)$ & & $6^{2}$ & $-6^{2}$ & $+\phi^{2}$ & +66 & $+\infty^{2}$ & $-\omega^{2}$ & $+\beta \phi$ \\
\hline
\end{tabular}

Fig. III $-4(a)$

Transitic 1 probabilities for $6^{2} p_{1 / 2} \rightarrow 7^{2} p_{1 / 2}$ transitions with misaligned electric and magnetic fields and imperfect laser polarization for (a) $\Delta m_{F}=0$ and (b) $\Delta m_{F}= \pm 1$ transitions. The first column gives the transition strength and subsequent columns list all the interference terms in the transition probability that are coefficients of $\sin \theta \cos \theta$ and can mimic PNC. The true PNC interference term is also listed. 


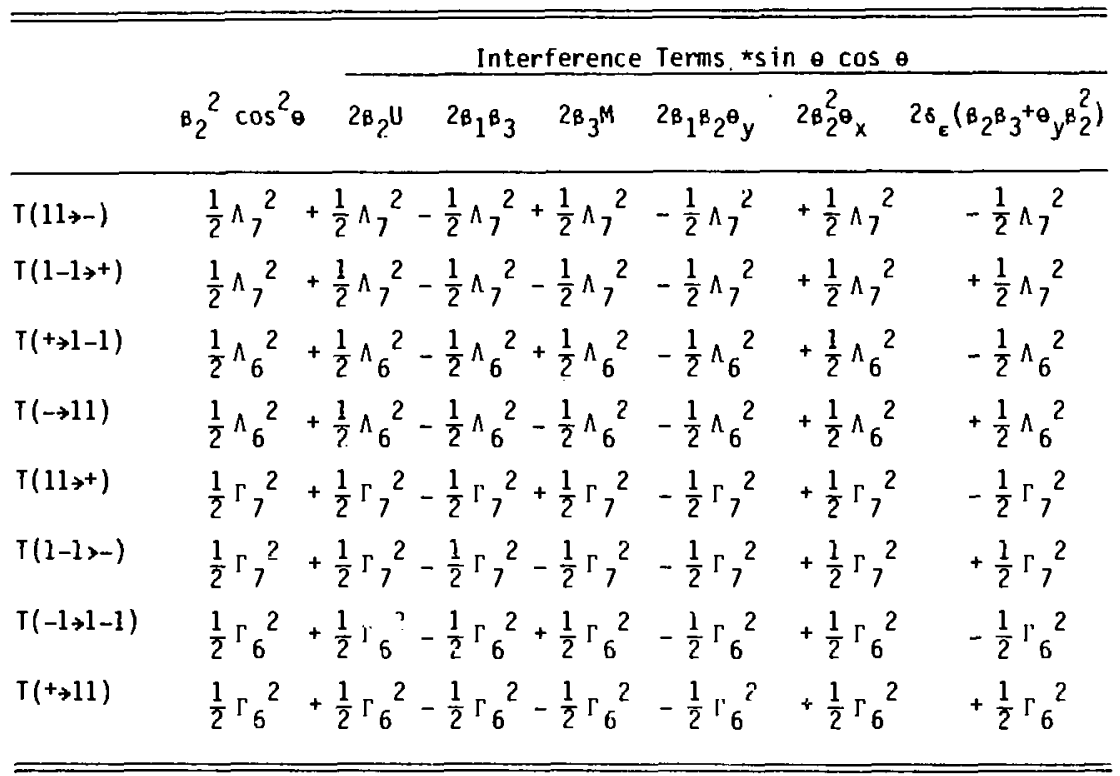

Fig. III $-4(b)$ 
they cancel in those two exactly overlapping lines. A small contribution to those terms comes in from the $|+\rangle|+\rangle$ transition but that is not on resonance and so the contribution relative to the line strength is reduced by a factor of 8 . Those two terms can then be eliminated quite satisfactorily by reversing the magnetic field and $k$ since both change signs with $B$ and $k$ wile the parity term does not. As sill be discussed in greater detait in Chapter $v^{*}$; the ${ }^{2}{ }_{2}^{2} \theta_{x}^{*}$ term can be kept * adequately small by adjustments made while data is being taken. The most difficult tems to eliminate satisfactorily are the $a_{1} a_{3}$ term and the $B_{1}{ }^{\theta} 2^{\theta} y$ term. The later enters in the asymmetry in the form, $\frac{\Delta E_{x^{\theta} y}}{E_{o y}}$ (the $\frac{E_{0 x^{A E} y^{\theta} y}}{E_{o y}^{2}}$ term is considered third order and dropped) and a separate experiment puts a limit or this quantity. The $\alpha_{1} \alpha_{3}$ term, which contribbutes to the asymetry as $\frac{a^{2}\left(E_{o x} \Delta E_{z}+E_{o z} \Delta E_{z}\right)}{a^{2} E_{o y}^{2}}$, can only be eliminated by a direct measurement. $E_{\partial z}, \Delta E_{x}$, and $\Delta E_{z}$ can be measured directly but there is no way that $E_{o x}$ can be independently determined. Instead, an experiment is done at a magnetic field of 700 Gauss where instead of the parity term $B_{2} U$ and the $a_{1} a_{3}$ term entering into the transition probability with approximately equa? weights, the $\alpha_{1} \alpha_{3}$ terms is enhanced and the $B_{2} U$ term is greatly supressed. This will be discussed in greater detail in Chapter $V$.

\section{Dilutions and Backgrounds}

There are two sources of dilutions in this experiment which will result in the measured asymmetry being somewhat smaller than $2 \mathrm{E}_{\mathrm{PNC}} / \mathrm{BE}$. 
These are incomplete line separation and finite signal to background ratio. The latter can also contribute to a false asymetry.

The problen of incomplete line separation has been mentioned previously. The hyperfine transitions cannot be completely resolved since the inhomogeneous line width due to Doppler broadening is approximately $1.6 \mathrm{GH} z$, whereas typical line separations at $4 \mathrm{KG}$ are only a few $\mathrm{GHz}$ (see Fig. II-7). Furthermore the lines may be broadened by the isotope shift in $T:$ of $1.27 \mathrm{GHz}$. Tl naturally occurs as $30 \%{ }^{203} \mathrm{~T} 1$ and $70 \%{ }^{205} \mathrm{Tl}$ but the experiment can be done with isotopically pure ${ }^{205} \mathrm{Tl}$ to remove this source of line broadening. The consequence of the line overlap is that at a given frequency there are contributions from 1 ines of opposite asymetry.

The transitions listed in Fig. II-7 or III-4 can be grouped into two categories. The $\Delta m_{F}=0$ transitions have a leading term of $B_{2}^{2} \sin ^{2} \theta$ and a PNC interference term of negative sign while the $\Delta m_{F}= \pm 1$ transitions have $B_{2}^{2} \cos ^{2} \theta$ as the leading term and the interference is positive. Let $Q_{j}(0)$ be the coefficients of the $\Delta m_{F}= \pm 1$ transitions and $Q_{j}(90)$ be the coefficients of the $\Delta m_{F}=$ 0 transitions. (These coefficients are listed in Fig. III-4.) Then

$$
\begin{aligned}
T^{g 0}(\omega) & =\sum_{i} Q_{i}(90) P\left(\omega-\omega_{j}\right) \\
T^{C}(\omega) & =\sum_{i} Q_{i}(0) P\left(\omega-\omega_{i}\right) \\
P\left(\omega-\omega_{j}\right) & =\begin{array}{c}
\text { Doppler broadened lineshape } \\
\text { centered at } \omega_{i} .
\end{array}
\end{aligned}
$$


At a given frequency, the total intensity will be given by

$$
T^{\theta}=T^{0}(\omega) \cos ^{2} \theta+T^{90}(u) \sin ^{2} \theta
$$

and the measured asymmetry will be given by

$$
\Delta^{\theta}=\frac{2 \varepsilon_{\text {PNC }}}{B E} \quad \frac{T^{0}(\omega)-T^{90}(\omega)}{\left(T^{0}(\omega) \cot \theta+T^{90}(\omega) \tan \theta\right)}
$$

as compared with the somewhat simpler expression in (II.28). A computer program is used to generate the lineshape curves and to $f$ ind the optimal value of and $B$ to minimize the running time of the experiment. Computer generated curves of the lineshape in the $F=1 \rightarrow F^{\prime}=$ 1 transition are given in Fig. III-5 with a magnetic field of $4 \mathrm{KG}$ for TI with natural isotopic abundance and for isotopically pure ${ }^{205} \mathrm{~T}$. Two curves are shown for each case: $T^{0}(\omega)$ and $T^{90}(\omega)$, and two optimal transitions are indicated, one to measure a positive, the other a negative asymmetry. For pure ${ }^{205} \mathrm{Tl}, \mathrm{T}^{\theta}(\omega)$ is also shown.

As Fig. III-5(b) shows quite dramatically, another consequence of the finite line width is that at $\theta=34^{\circ}$ where the running time as defined in equation $I I .29$ is minimized for $B=4 \mathrm{KG}$, the resonance is relatively flat for many $\mathrm{GHz}$ across the entire hyperfine spectrum. In order to stay on resonance, one must look at the signal with $\theta=0^{\circ}$ and $\theta=90^{\circ}$, since both are very sharp functions of $\omega$. The maximum asymmetries occur where

$$
R=\frac{T^{90}(\omega)}{T^{0}(\omega)}
$$

is a local maximum or a local minimum. Once the laser is close to a point of maximal asymmetry, a feedback loop that maximizes or 

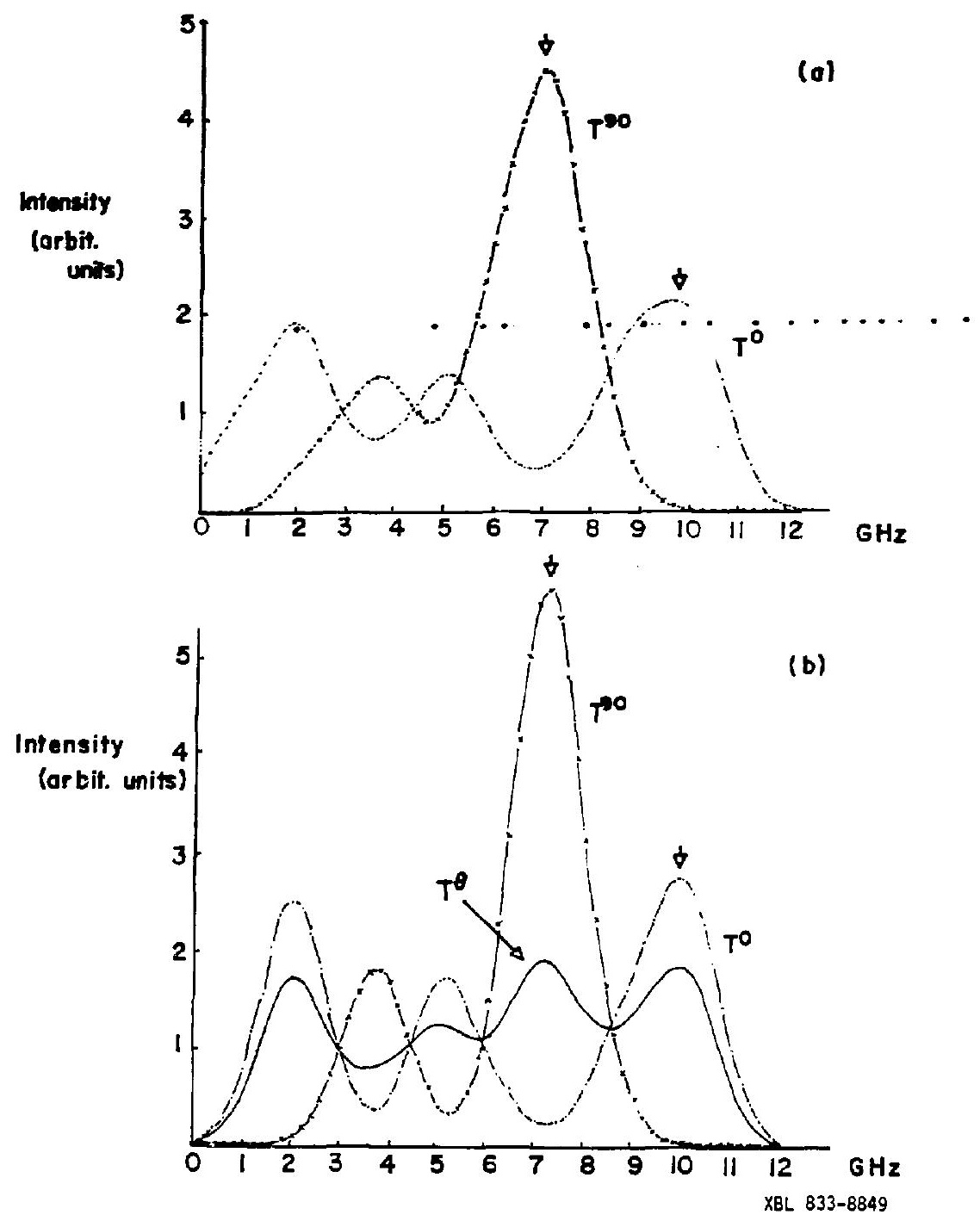

FIg. III-5 
Fig. III-5. Computer generated curves showing the $6^{2} P_{1 / 2}, F=1$ $7^{2} \mathrm{P}_{1 / 2}, \mathrm{~F}^{\prime}=1$ transition lineshape as a function of laser frequency for (a) Matural $\mathrm{T}=30 \% 203 \mathrm{~T}$ + 70\% 205 $\mathrm{Tl}$ and (b) Isotopically pure 205T). Lineshapes are shown for both $\theta=0^{\circ}$ and $\theta=90^{\circ}$ ( $\mathrm{TO}^{\circ}$ and $\mathrm{T}^{90}$ ) to exhibit both $\Delta m F=0$ and $\triangle M F F= \pm 1$ lines. In (b), $T^{\theta}$ for $\theta=34^{\circ}$ is aiso shown. 
minfmizes $\mathrm{R}$ can hold it there. During the experiment, tuning data are taken at $\bullet=0$ and $\bullet=68^{\circ}$ in addition to parity data at $\bullet= \pm 34^{\circ}$. Maximizing $T^{90} / T^{0}$ is equivalent to maximizing $\mathrm{T}^{68} / \mathrm{T}^{0}$, and the laser is automatically kept on resonance.

A finite background in the experiment will also dilute the measured asymmetry. . Consijder (II.28) where. $T(*)$ is, rep laced by $T^{*}+\ldots$. . . $B$ where $B$ is the total background and is assumed for the moment not to vary with the reversal of $E$ or $\theta$. The measured asymmetry becomes

$$
\begin{aligned}
\Delta & =\frac{\left(T^{+}+B\right)-\left(T^{-}+B\right)}{T^{+}+B+T^{-}+B} \\
& =\frac{T^{+}-T^{-}}{T^{+}+T^{-}} \frac{T}{T^{+} B} .
\end{aligned}
$$

The true asymmetry has been diluted by the ratio of the signal to the signal plus the background. Currently the background in the experiment is quite large $\left(\frac{T}{T+B}=.8\right.$ ) and improvements are underway to reduce it to a more acceptable level.

A potentialiy greater problem that the background can cause resutts from any dependence of the background on the direction of $E$ or of $\theta$. There are several sources of background which can be classified as molecular background, direct scattered light background, and indirect scattered light or electric field background. The first two are relatively harmless. The molecular background is a broad background that is independent of the electric field and is thought to be due to sticky collisions between thallium atoms inducing electric dipole transitions between the $6^{2} \mathrm{P}_{1 / 2}$ and $7^{2} \mathrm{P}_{1 / 2}$ states. ${ }^{49}$ This background is typically a factor of 10 below the signal level of the transition. Direct scattered light is due to laser photons at $293 \mathrm{~nm}$ 
being bounced into the detectors. This background is extremely small because $293 \mathrm{~nm}$ light is effectively blocked from the detectors by a Phthalic acid filter. 50 There is some fluorescence in the quartz cell window and detector window which downshifts the light into the passband of the detection system, but the scattered light contribution to the background is still less then $2 \%$ of the signal.

The electric field background is at present the mos troubiesome because it is large (almost $15 \%$ of the total signal) and it has a strong dependence on $E$ and a somewhat weaker dependence on $\theta$. This background is thought to be caused by scattered laser light striking the tantalum electrodes inside the cell that are used to produce the electric field. Electrons are ejected from the metal, accelerate in the few hundred volts/cm field, and collide with and excite Il atoms. This background is profoundly affected by the magnetic field since then the electrons spiral around the field lines with orbits of only $.1 \mathrm{~mm}$ and are accelerated by the Lorentz force either towards or away fram the detector, resulting in a strong dependence in the background with the sign of $E$. Since the amount of scattered light varies with $\theta$ depending on whether the polarization is in or out of the plane of incidence with the scattering surface, there is a component to this background that varies with $E$ and $\theta$ just as the PNC interference. The background can be reduced by taking data at two frequencies where the asymmetry is of opposite sign and the ratio of signal to background is the same. However, great care must be taken that the laser beam does not move as the frequency is scanned since the background would then change and the subtraction would not be adequate. This frequency 
subtraction has been tried but the size of the background and the asymmetry it produces (typicaliy 5 - 10 times the size of the expecter PNC 3symetry) require a more drastic solution. The work function of tantalun is approxinately $4.13 \mathrm{ev}^{51,52}$ which is below the $4.24 \mathrm{eV}$ of the laser photons. Electrons can therefore be ejected rather easily. A new cell is presently being made with Ni electrodes. The work function of $\mathrm{Ni}^{\circ}$ is $5.01 \mathrm{ej}^{53}$ so it will take a multiphotion absorbtion to remove electrons from the surface of the metal and the electric field background should then be eliminated entirely.

At present experimental limits exist on all of the false asymmetries except for the $a_{1} a_{3}$ interference. The large background asymmetry prevents us from being able to reduce the limits below the 4-5 $\times 10^{-6}$ level. An explanation of how the systematics have been measured and upper limits on their values will be presented in Chapter $V$ after a detailed description of the experiment which follows in Chapter IV. 


\section{APPARATUS}

Figure IV-I presents an overview of the apparatus for this experiment. The individual components will now be described in detail, as well as results from several tests used to calibrate the system.

\section{Laser}

A dye-laser oscillator-amplifier" syśtemi generätès "the" high" intensity $293 \mathrm{~mm}$ light needed to drive the $6^{2} \mathrm{P}_{1 / 2} \rightarrow 7^{2} \mathrm{P}_{1 / 2}$ thallium transition. A frequency stabilized $\mathrm{cw}$ dye laser (Coherent Inc. Model 599-21) pumped by an argon ion laser (Coherent Inc. Mode] CR-6) is used as an oscillator and is amplified by three dye amplifiers Dumped by the doubled output of a Q-switched Nd: YAG laser (Quanta Ray DCR OSCillator). Details of the laser are described elsewhere ${ }^{54}$ and a schematic is shown in Fig. IV-2. For maximum efficiency at $585 \mathrm{~nm}$, the amplifier chain is operated with Rhodimine 610 (Exiton) dissolved in methanol in concentrations of $145 \mathrm{mg} / \mathrm{l}$ in the first two stages and $25 \mathrm{mg} / 7$ in the last stage. The laser produces $10-15 \mathrm{mj}$ of $585 \mathrm{~nm}$ light per $5 \mathrm{nsec}$ pulse at $17 \mathrm{~Hz}$ in a 100 MHz bandwidth. The beam is approximately $3 \mathrm{~mm}$ in diameter and is nearly diffraction limited.

The output of the amplifier chain goes unfocused into an angle tuned KDP crystal, (Cleveland Crystals $12 \mathrm{~mm} \times 12 \mathrm{~mm} \times 30 \mathrm{~mm}$ ); and $293 \mathrm{~nm}$ UV light is generated with $30 \%$ efficiency. The UV is horizonally polarized and is separated from the visible light with a fused silica Brewster angle prism (Quanta Ray) which deviates the beam $90^{\circ}$. The energy of the UV beam is monitored at this point with a pyroelectric 


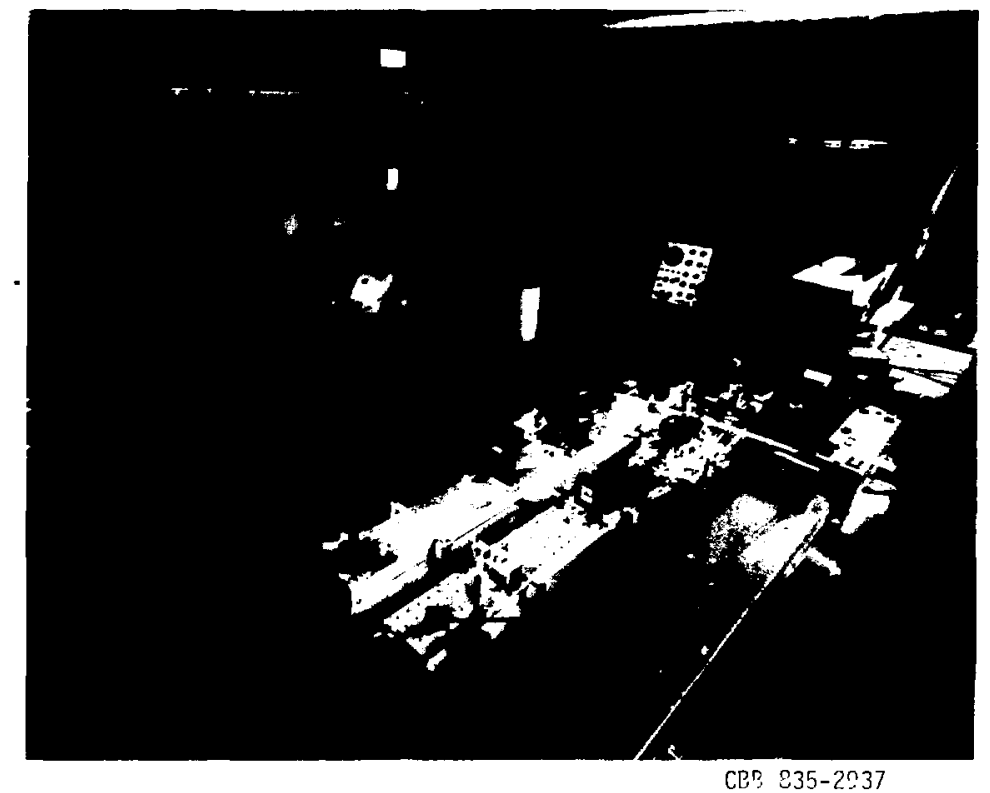

Fig. IV-1. Overview of the apparatus showino lasers iri the forearound and the magnet with the vacuum can installed in the center. 


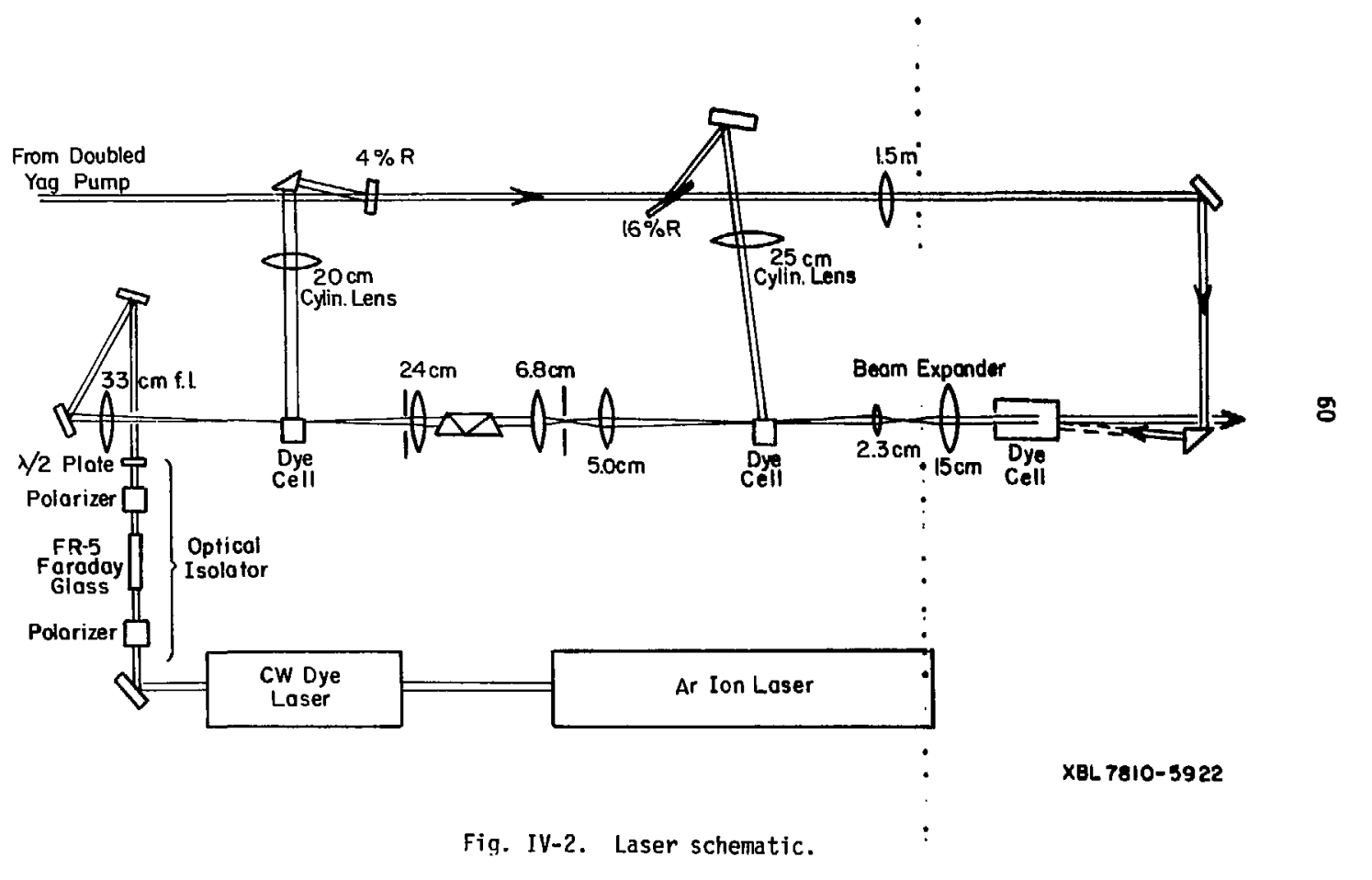


joulemeter (Holectron 3-3). Two mirrors then steer the bean so that it lines up with the polarizing elements and the thallium cell. The mirrors are hard coated for $P$ polarization and the extinction of the linear polarization measured after the mirrors and before the Pockels cells, using a Glan-air prism and a photodiode, is better than $10^{3}$.

To look for parity nonconservation, linearly polarized light. is .. . needed, where the angle between $\hat{\varepsilon}$ and the magnetic field must switch from to to $\rightarrow$. It is also very convenient to have the light polarized parallel to $B$ and approximately perpendicular to $B$ in order to stay on resonance, as was described at the end of Chapter III. With two Pockels cells in series, each acting as a fixed half-wave plate, one has four options for the direction of the outgoing linear polarization when the effective fast axes of the Pockels cells are sequentially at $a$ and $\beta$ :

\begin{tabular}{ccc} 
PCII $(\alpha)$ & PCI (B) & Polarization \\
\hline OFF & OFF & HORIZONTAL $=0^{\circ}$ \\
ON & OFF & $2 \alpha$ \\
OFF & ON & $2 \beta$ \\
ON & ON & $2 B-2 \alpha$.
\end{tabular}

This is shown diagramatically in Fig. IY-3. When $\hat{\varepsilon}$ is at \pm 0 with respect to the magnetic field, the parity experiment collects data. The ratio of the signal size when $c$ is at $0^{\circ}$ to that at 20 with respect to the field is used for the laser tuning. Thus we choose $\alpha=\theta$ and $B=0 / 2$ to get the desired choices for the linear polarization. 

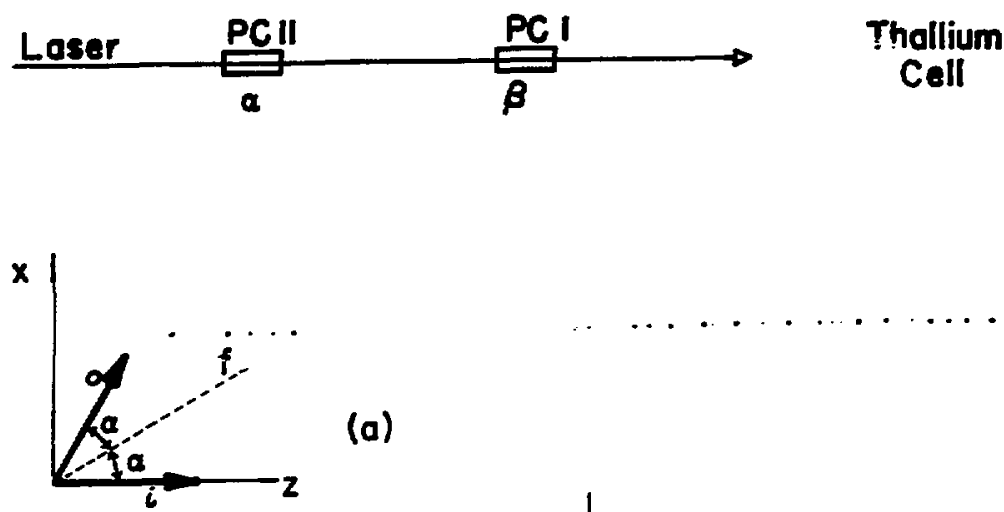

(a)

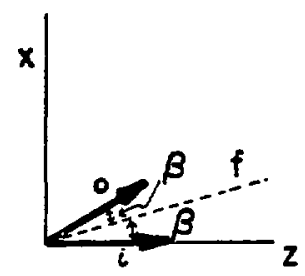

(b)

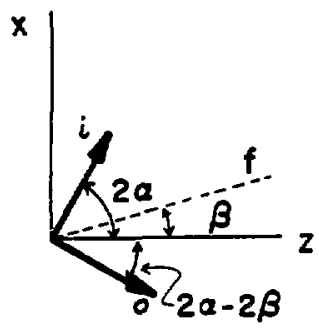

\section{(c)}

XBL $833-8850$

Fig. IV-3. Possible orientations of laser polarizations; $i$ refers to incident, o refers to outgoing. If no voltage is applied to the PC then the outgoing polarization is the same as the incident polarization. (a) Shows the effect of PCII acting as a $\lambda / 2$ plate with fast axis at angle $\alpha$. (b) Shows the effect of PCI acting as a $\lambda / 2$ plate with fast axis at angle $\beta$ when no voltage has been applied to PCII. (c) shows the effect of PCI when voltage has been applied to PCII. The four possible linear polarizations are described in the text in IV.I. 
It is possible that the magnetic field is at a small angle with respect to the horizontal axis determined by the laser polarization after the doubling crystal. In this case we choose $\alpha=0$ and $a=(0+\phi) / 2$ by rotating Pockels cell I until to is symetric about $B$. The atoms themselves are used in this final fine alignment of the linear polarization as shall be explained later.

The Packels cells used in the experiment are $10 \mathrm{~mm}$ dianeter $\times 30 \mathrm{~mm}$ long KD*P ( $C$ leveland Crystals QX 1020) where the crystals are specially selected for high transmission in the UV. With peak laser powers in excess of 5 Watts $/ \mathrm{cm}^{2}$, the Pockels cells suffer considerable surface damage and some bulk crystal damage. Running the laser with 2-3 mj/pulse incident upon them, the Pockels cells last several hundred hours before needing to be repolished due to surface damage. The crystals are sealed in desiccated housings with AR coated windows. Attempts were made to use index matching fluid to reduce the scattering and reflection losses from the crystal surfaces, but the damage threshold of the crystals was greatly reduced. After the Pockels cells, the crossed polarizer rejection is better than $10^{3}$. Both Pockels cells are mounted in modified mirror mounts with additional vertical and horizontal translation stages and a retational adjustment so that the crystal can be rotated about the longitudinal axis for fine adjustments of the polarization angle while its position remains unchanged. The $\lambda / 2$ voltage for the Pockeis iells is approximately 1800 volts applied to two cylindrical ring electrodes. The voltage pulse is $1 \mathrm{msec}$ long with a 100 ysec rise time. 
The beam then enters the vacuum can through a quartz window and passes through the cell. At the far end of the cell a uY mirror ref lects the baam back on itse]f and back out the front window. The mirror can be blocked by a beam stop, upoiz a command from the computer, so that data can be taken with ard without the mirror reflecting the beam. By replacing the mirror with a quartz window, the birefringence of the can windows and cell can be measured on the far side. This was attempted and on the far side of the vacuum system the crossed polarizer extinction was still found to be better than $10^{3}$.

The position of both the initial beam and the beam reflected from the mirror at the back end of the can is monitored with a $5 \mathrm{~mm}$ element quadrant detector (Molectron Q-E). A reflection off the surface of the Pockels cell can be used to detect the position of the incident beam and a fraction of the beam returned from the back mirror can be used to sense the position of the beam at the far end of the cell. Using these two references the position of the beam inside the cell can be held fixer so that angular deflections are less than $1 \mathrm{mrad}$ and the position of the beam changes by less than .1mm. The backup circuit for the quadrant detector is shown schematically in Fig. IV-4. The normalized sums and differences which indicate movement of the beam across the quadrant detector are displayed digitally and if the beam moves, the direction and size of the motion is indicated. The drifts in the laser beam position are slow. Typically the beam drifts a few mrad in several hours due to drifts in the YAG and $\mathrm{Ar}^{+}$ laser beams, and adjustments to keep the beam in a fixed position are 


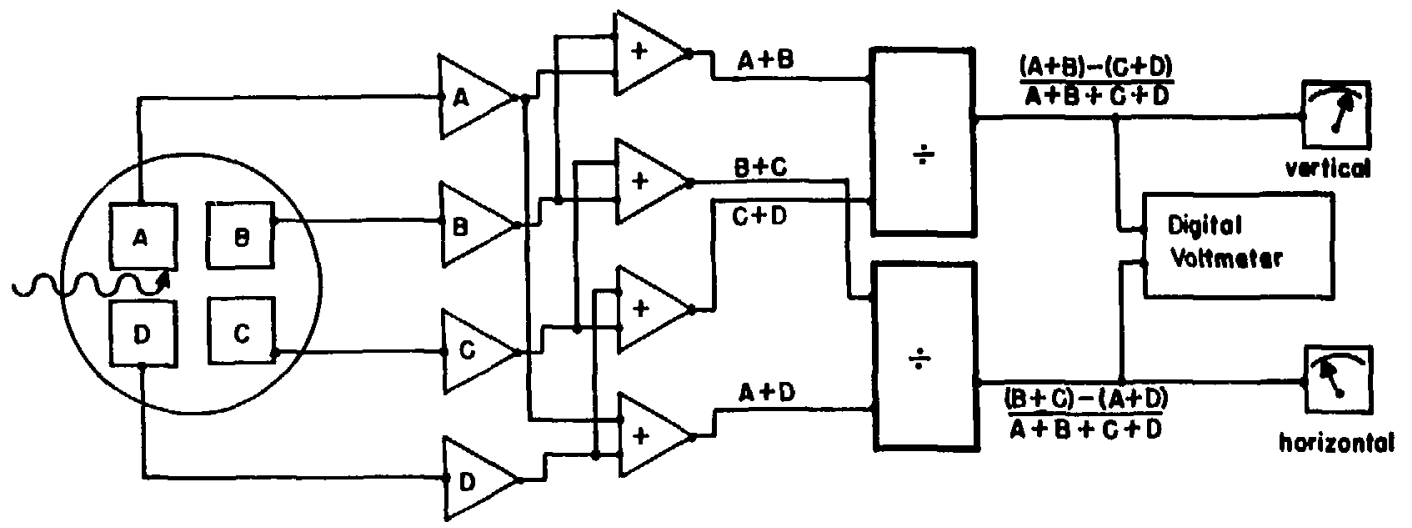

Fig. IV-4. Backup circuit for quadrart detector. Signals from the 4 pads are amplified, summed, and then the appropriate differences of the sums are taken and normalized to the total incident intensity to give horizontal and vertical deflection information. 
done manually using the steering mirrors in front of the Pockels cells. A diagram of the path of the laser is shown in Fig. IV-5.

The frequency of the UV laser is determined by the output of the Cw dye laser, which is actively stabilized to $5 \mathrm{MHz}$. However, drifts in temperature as the laser warms up can result in frequency drifts of many MHz. To insure that the laser remains at the peak of the resonance, a computer controlled feedback loop is used. Interspersed with the parity data, every 17 th and 18 th laser shot is devoted to tuning data. The ratio

$$
R=\frac{T^{2 \theta}(\omega)}{T^{0}(\omega)}
$$

sormed, averaged over 16 measurements and compared to its previous value. If it has decreased then the computer assumes the laser has drifted of $\mathrm{f}$ resonzence and adjusts the laser frequency, by means of a DC voltage, in $50 \mathrm{MHz}$ steps. Always the current valve of $R$ is compared to the previous valve of $R$. If $R$ decreases, the step direction is reversed; if $R$ increases, the step direction remains the same.

The computer also scans the laser. Data are taken at two fixed frequencies (see Fig. III-5) and the laser jumps between them once per second. Here, $R$ is maximized for one transition and the jump frequency is fixed by changing the $D C$ voltage that the computer applies to the external jack of the control box of the laser by a fixed amount. The computer can also scan the $\mathrm{cw}$ laser across the entire hyperfine spectrum; a technique used to do diagnostic tests on the experiment. Great care must be taken when the laser frequency is changed however, to avoid systematic beam motion. The frequency of the laser is changed by tilting a $1 \mathrm{~mm}$ quartz flat mounted on a galvanometer and 


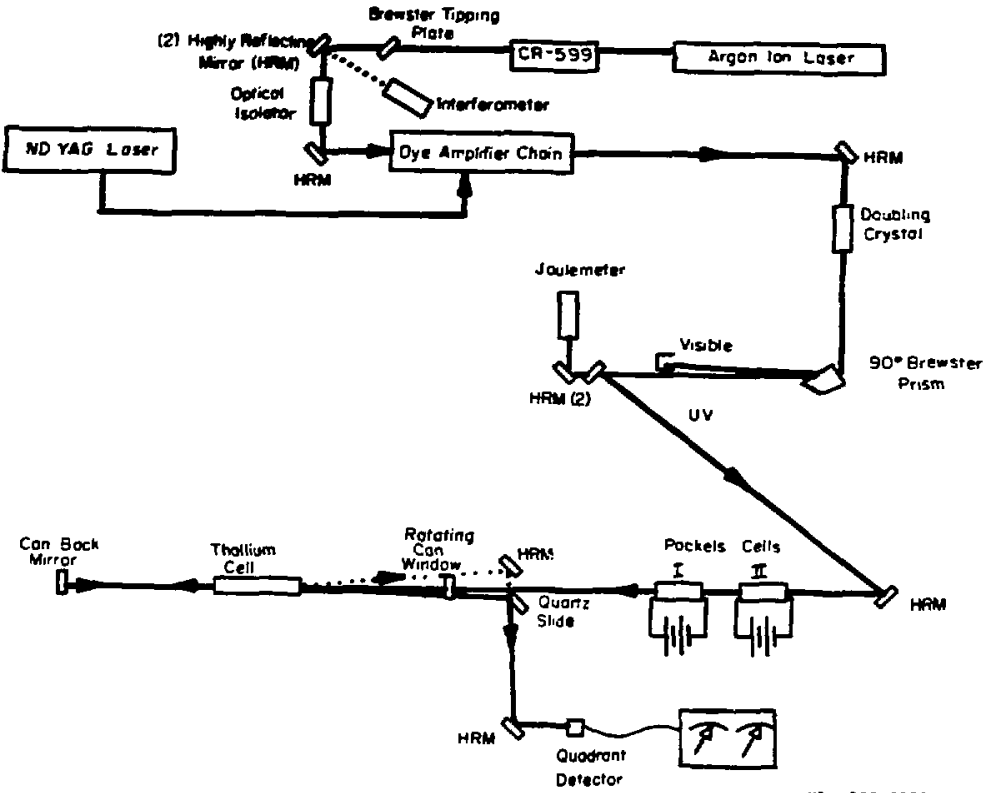

XBL $833-8852$

Fig. IV-5. Schematic diagram of the path of the laser beam. 4\% reflections off of surfaces are shown as dotted lines. 
held at close to Brewster's angle in the laser cavity. As the plate tilts, the optical path length changes and correspondingly, so does the frequency. Unfortunately, the bean also moves. It is important that the background not change in a systematic fashion for data taken at two different frequencies where the parity interference is of an opposite sign, since this could show up in the data, so a second galvanometer driven quartz flat held at Brewster's angle was added outside the laser cavity. It tilts in the opposite sense as the intracavity plate and cancels any beam motion. The voltage driving this second galvanometer is extracted from the laser control box and comes from the curcuit that drives the laser galvanometer. An adjustable gain allows for differences in the galvanometer sensitivities. The quadrant detector is used to verify the absence of beam motion as the frequency of the laser is scanned over far greater ranges $(60 \mathrm{GHz}$ in the UV) than the frequency chop of $2.8 \mathrm{GHz}$ in the experiment. 2. Thallium Cell and Oven

In order to produce the necessary vapor pressure of .1 torr, the thallium for the experiment must be heated to $1000^{\circ} \mathrm{K} .55$ To accomplish this the thallium is placed in a quartz cell and surrounded by a nickel oven. The oven is, in turn, surrounded by a vacuum can and the entire apparatus, including a separate pumping system for the thallium cel1, sits in the 6-1/4" gap between the pole pieces of a $5 \mathrm{KG}$ electromagnet.

The cell is shown in Fig. IV-6. The cell body is a $48 \mathrm{~mm}$ 0.D. quartz cylinder with $2.5 \mathrm{~mm}$ walls. A 2-3/4" stem contains the thallium 


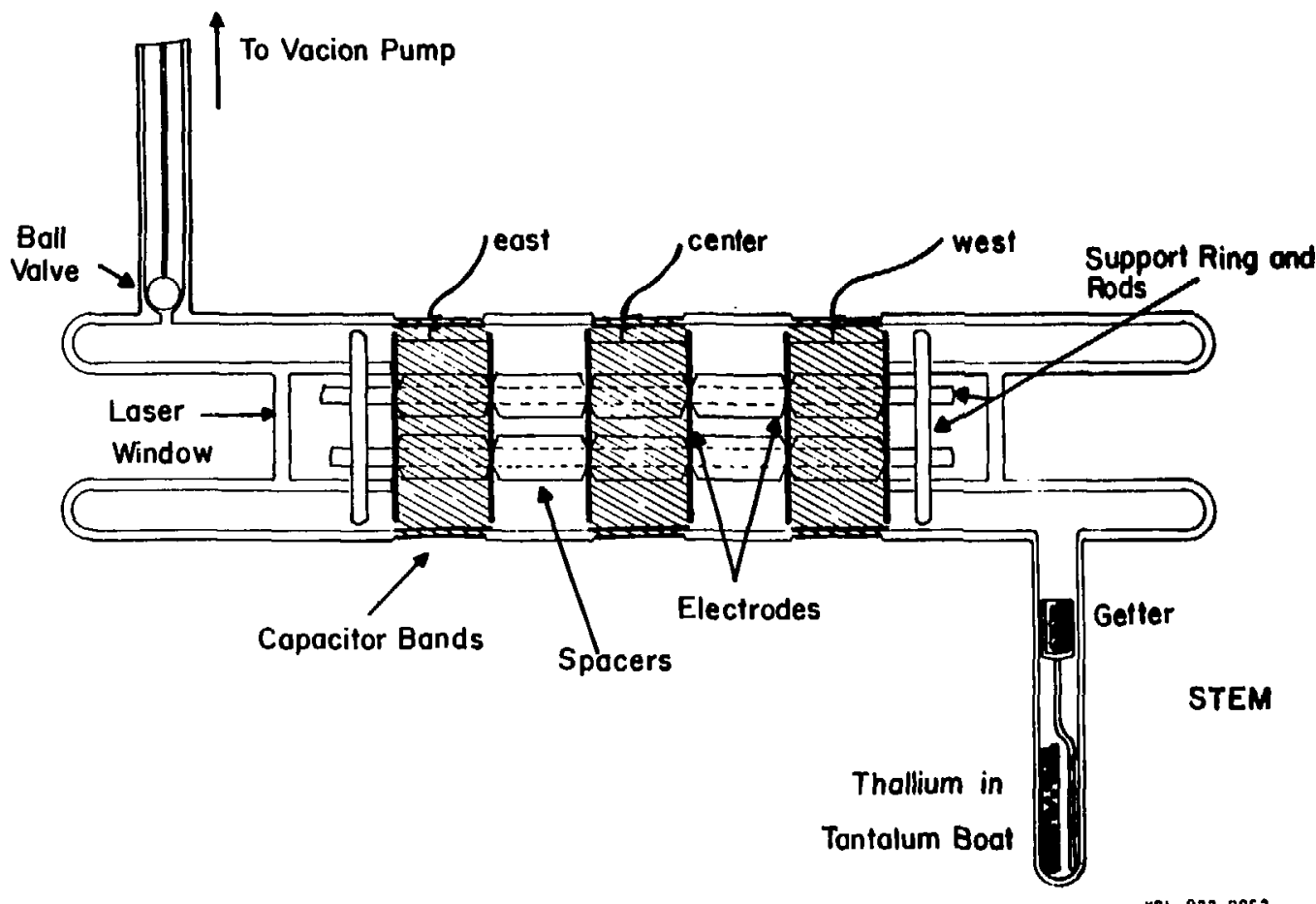

Fig. VI-6. 
Fig. IV-6. Thallium cell. Shaded regions show placement of bands both inside and outside the cell for capacitive coupling. Unshaded regions between bands are the interaction regions viewed by the detectors. Support rods and spacers to position electrodes are also shown. 
reservoir and a quartz cup holding getter. The stem part of the cell sits in a separate oven that is typically $130^{\circ} \mathrm{F}$ cooler than the main oven. The stem temperature determines the thallium density and is the coolest portion of the cell except for the pump out manifold. The cell hangs from its pump out manifold which is connected through the top of the vacuum can to a Vac-ion pump (Varian). A ground glass ball valve seals the cell during data taking but can be lifted by applying current to a copper wire solenoid placed around the top part of the ball valve (outside the vacuum system) which is made of steel plug vacuum sealed in quartz. This allows the cell to be purged of impurities periodically.

The windows of the cell through which the laser passes are Suprasil quartz disks (Amersil), 1/8" thick, in re-entrant holders. This allows the windows to be annealed after they are sealed to the window holders to reduce birefringence, and the final cell seal attaching the window holder to the end of the body is made far enourn from the window not to introduce any additional stress. Great care is taken during construction so that the window surfaces end up parallel, usually to better than $2 \mathrm{mrad}$.

The cell contains 6 metal plates which form the electrode structure and provide the necessary electric fields for the experiment. In the past, these plates have been tantalum and in the current cell under construction they are nickel. Care must be taken in the choice of materials put in the cell that they react neither with thallium nor with quartz at elevated temperatures. The electrodes are $4 \mathrm{~cm}$ in diameter and $.040^{\prime \prime}$ thick and have a $12 \mathrm{~mm}$ hole 
on center for the laser bean to pass through. The hole distorts the field in the interaction region somewhat. This can be calculated using a relaxation method to solve Laplace's eqn. in cylindrical coordinates. It turns out that the field gradients in the portion of the interaction region of interest are negligable. There is a diminution of the field from its expected valve and this must be accounted for in estimating the field in the region. Figure IV-7 shows the profile of the electric field in the region between the electrodes. Figure IV-8 shows the spacing and electrical connections between the electrodes. The outer two electrodes are present to smooth the field lines in the interaction region, as well as to act as baffles to block scattered laser light. The electrodes are supported on 4 quartz rods and separated by quartz spacers ground to a precision of .001". The ends of the window holders (see Fig. IV-6) serve as supports for the rods and provide a flat surface ground parallel to the windows for aligning the electrodes so that the plane of the electrodes is parallel to the plane of the windows. The electrode assembly is not a tight fit in the cell however. Several thousandths of an inch must be left as clearance to accommodate thermal expansion of the plates.

By far the most technically difficult part of the cell construction involves the electrical connection between the outside world and the electrodes inside the cell. Many methods have been tried in the past ${ }^{49,56}$ with varying measures of success. In this experiment, two methods have been used. The first irivolved making electrical feedthroughs by collapsing quartz onto molybdenum foil. 


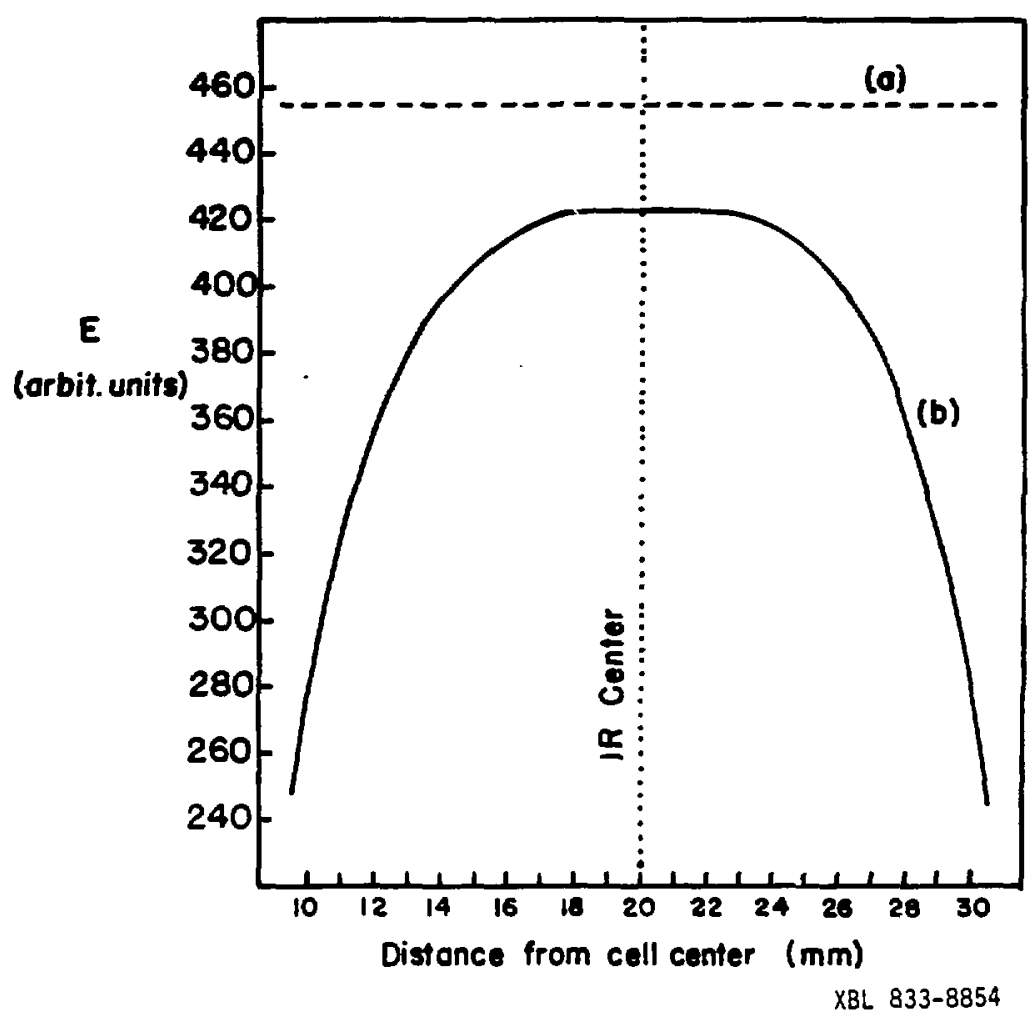

Fig. IV-7. (a) Ideal electric field in interaction region. (b) Calculated longitudinal electric field in interaction region taking into account holes in electrodes. The center of the interaction region is indicated. 


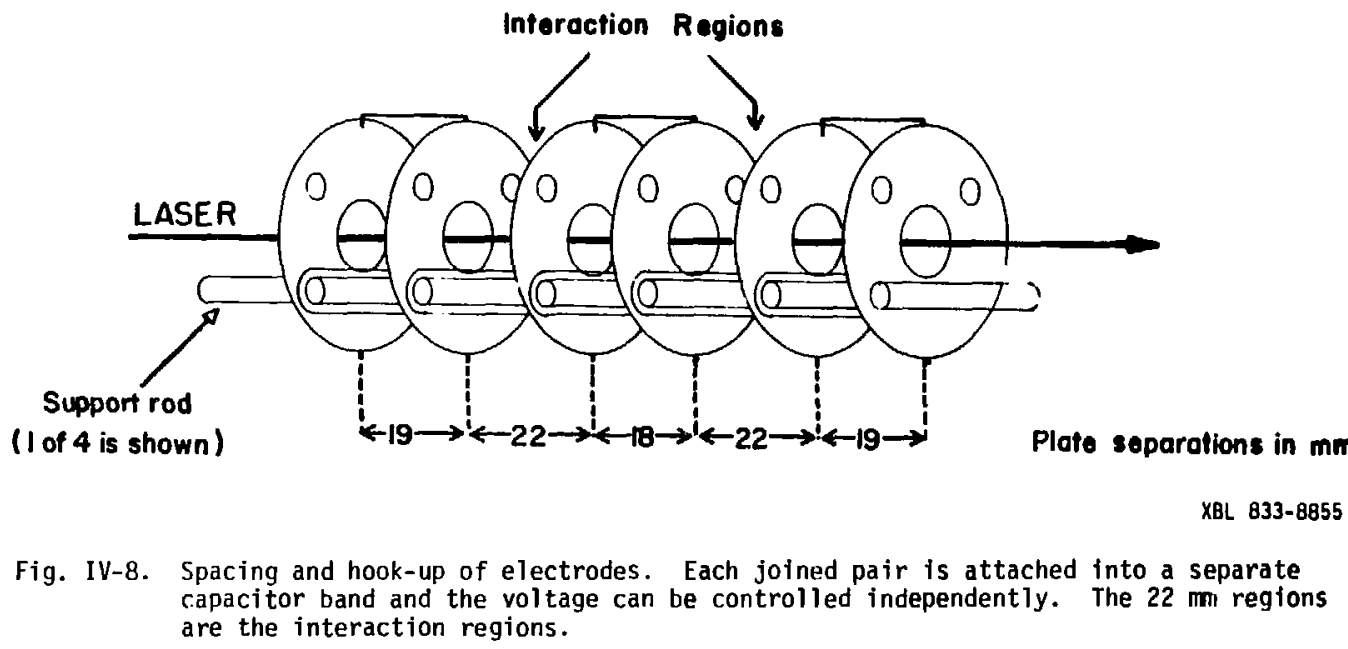


They were then sealed into the cell wall and made a direct electrical connection between the electric field plates and the excernal world. The feedthroughs were extremely time consuming and difficult to make and very delicate. Furthermore, wires connecting the feedthroughs to the electrodes caused troublesome off axis components of electric field in the interaction regions. For the latest celis a new design was proposed which has proved very successful. Instead of being brought in directly, the electric field is capacitively coupled through the quartz using a short (< $100 \mathrm{nsec}$ ) pulse. This is accomplished with 3 pairs of $2 \mathrm{~cm}$ wide bands of thin tantalum oil, one inside and one outside the cell. The inner band is .010" thick and stiff enough to spring out against the cell wall; the cuter band is .004" thick and is held in place with a spring. The quartz cell wall between the bands is ground to $1.5 \mathrm{~mm}$ thickness to $10 . \mathrm{m}$ a seat for the bands and to increase the capacitance. The position of the bands is indicated in Fig. IV-6. The viewing regions are ke $i$ free and there are no connecting wires that need to pass near he interaction region. The one disadvantage of the new method is that we no longer know the field in the interaction regions as well. The capacitance between the tantalum bands is only about $40 \mathrm{pf}$ an $\mathrm{can}$ be shunted by stray capacitance to ground. Also, the capacitisce of the interaction region of a few pf couples with the band capacitance to act as a voltage divider. While these effects can be "eliably modeled and calculated, they can also be measured as will be further discussed when the electric field pulser is describec. Direct measurements of the capacitance of the bands and plates using an LC meter were made 
before the windows of the cell were sealed on. These were then compared with valves inferred from calibration experiments to determine the field in the interaction region, and the agreement is excellent.

The cell is surrounded by a nickel main oven and a stainless steel stem oven connected to the main oven by a 1/32" walled tube. The oven is shown in Fig. IV-9. Nickel was choosen because it does not affect the quartz at high temperatures in a good vacuum. Stainless steel had been used previously but fogged viewing optics and cooler parts of the cell when used in a good diffusion pump vacuum of $10^{-5}-10^{-6}$ mini $\mathrm{Hg}$. The nickel remains clean at high temperatures in a good vacuum and the experimental temperatures are well above the Curie point for nickel so its magnetic properties are not a problem. A good vacuum helps keep the cell and can windows ciean and free oí oil so that they are less susceptible to burning due to the laser.

The heater elements are made of .030 " tantalum wire wound helically, and arranged around the oven to cancel any induced magnetic fields. The heaters are held in ceramic spacers to prevent them from shorting to each to eac other or to the cell, but care must still be taken in affixing them since the magnet's field exerts considerabie force on them. The main oven heaters run at 7 amps ac and the stem heaters at 5.5 amps. The temperatures of the ovens are measured with chromel-alumel thermocouples placad in the bodies of the ovens and read by a digital voltmeter. Calibration curves are taken from the AIP Handbook. ${ }^{57}$ One nickel and three titanium heat shields surround the oven to reduce radiation losses. The oven has two holes in it, 


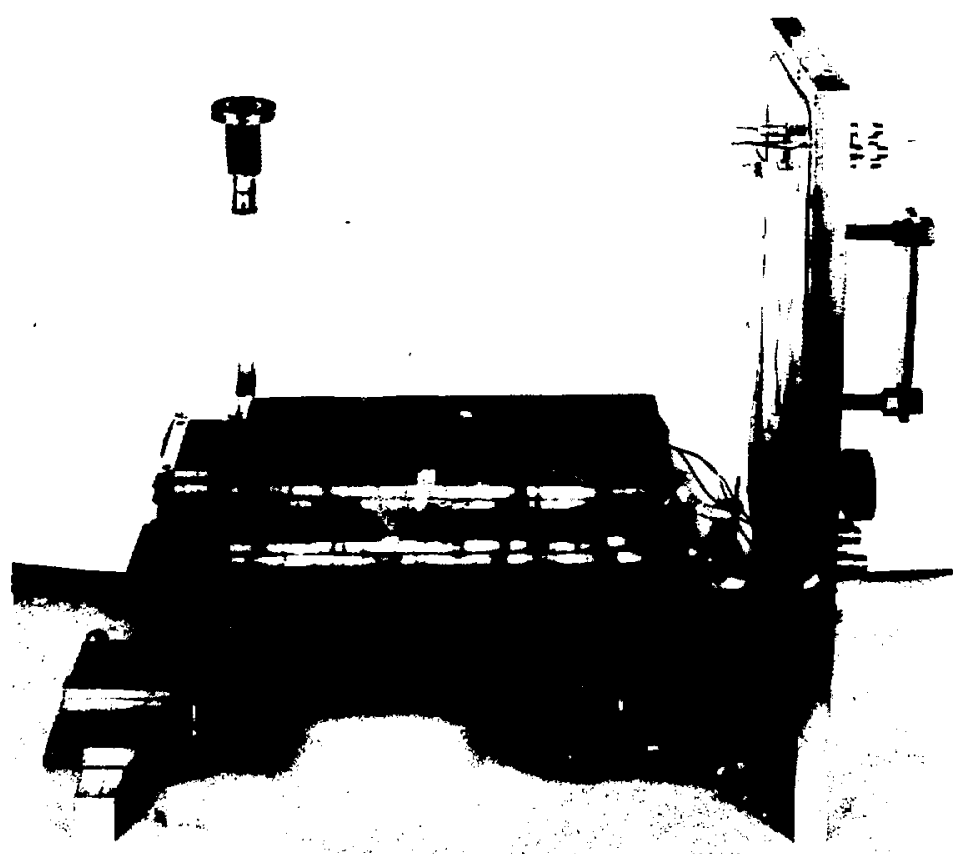

CBB 833-2939

Fig. IV-9. Oven attached to vacuum can flange for installation. The heat shields are removed to expose the heaters. 
1-1/2" in diameter, one on top and one below, with which to $v^{\circ} \mathrm{ew}$ the interaction regions. These holes are covered with 1/4" quartz windows to reduce heat loss due to black body radiation from the oven.

The oven is enclosed in an aluminum vacuum can. Tre entire oven and cell assembly is mounted on one end flange of the can to facilitate insertion and removal. To assemble, the entire vacuum system (minus the diffusion pump) is raised from between the pole tips of the magnet; the oven and cell, attached to the end flange, are slipped into place; the flange is bolted on, and the entire system lowered as showin in Fig. IV-10. The vacium system can be rotated about a vertical axis $\pm 10 \mathrm{mrad}$ and translated $\pm 5 \mathrm{~mm}$ from center while between the pole tips of the magnet. This is used for diagnostic tests of systematics, as well as to align the axis of the cell perpendicular to the magnetic field.

A water cooled electromagnet with $12^{\prime \prime}$ pole tips and a 6-1/4" gap provides a magnetic field of up to 5 Gauss for the experiment. The magnet is powered by a $2 \mathrm{Amp}, 5$ KWatt regulated suppty (Varian $V-2100 B$ ) designed for use in NMR applications and the current regulation is one part in 100,000. Figure IV-11(a) gives the langitudinal profile of the magnetic field as a function of position from the center of the pole tip and IV-11(b) gives B Vs. I, the calibration curve for the magnet. Both of these have been measured using an RFL Model $750 \mathrm{~A}$ gaussmeter with a Hall probe. The can is centered between the magnet pole tips so that the interaction regions are $\pm 2 \mathrm{~cm}$ from pole tip center and the field uniformity there is excellent. 


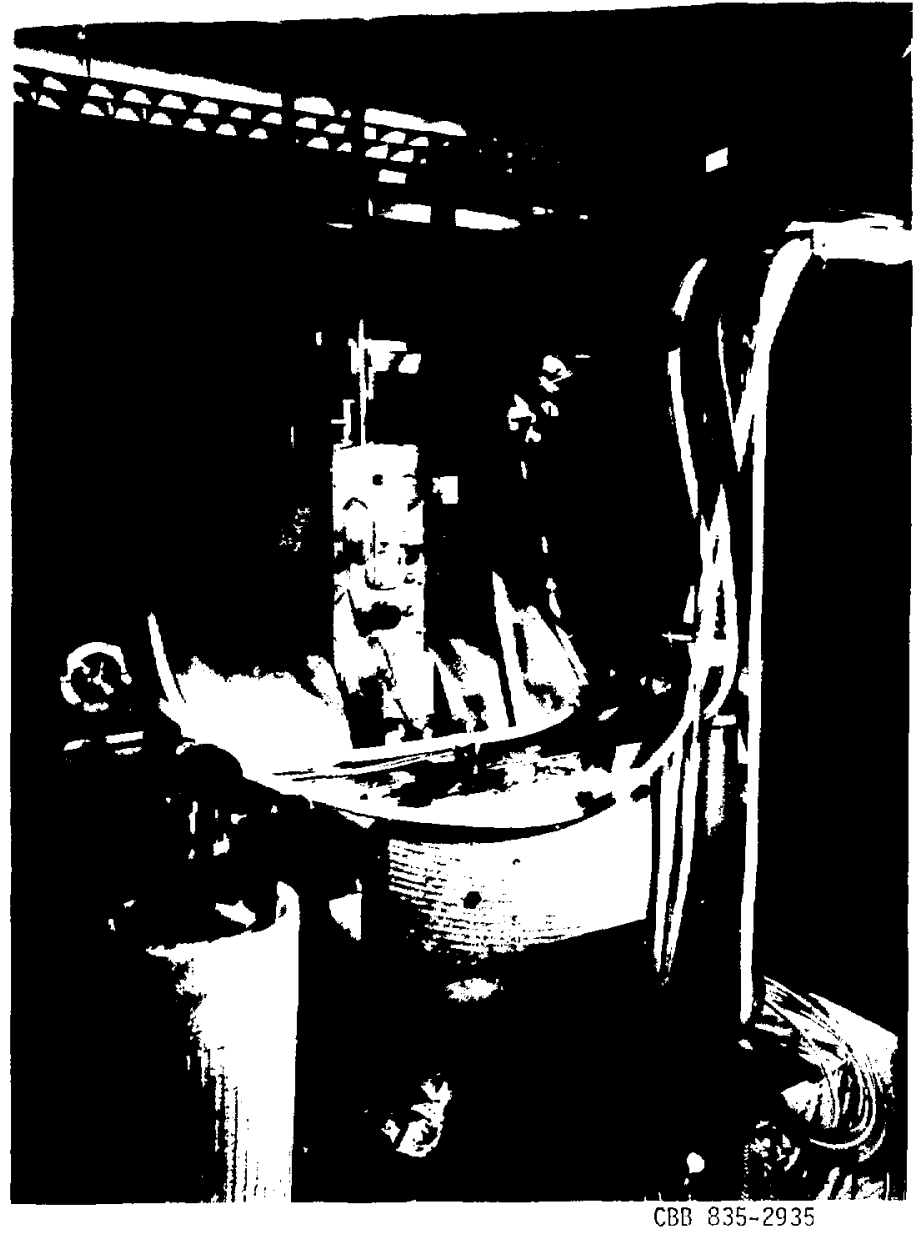

Fig. IV-10. The vacuum can is shown in the raised position in preparation for cell installation. 


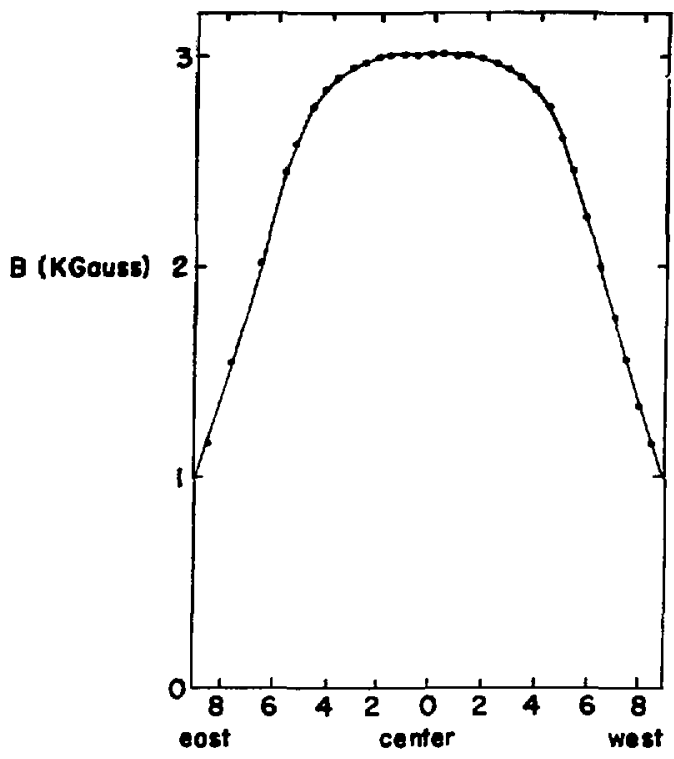

(a)

\section{Posifion from mognet center (inches)}

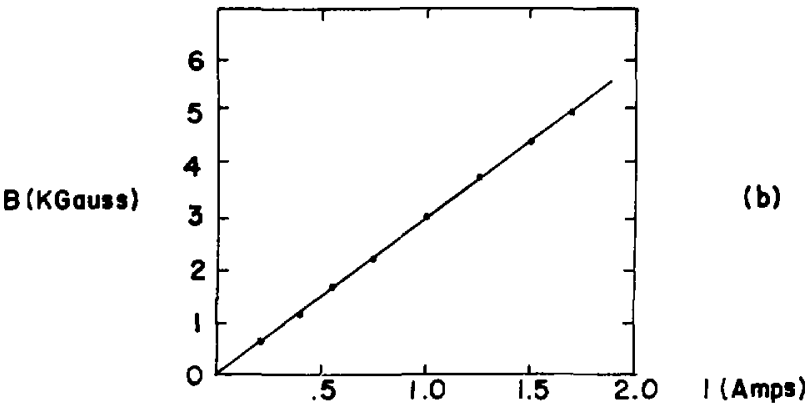

XBL $833-8856$

Fig. IV-11. (a) Profile of magnetic field along the $y$-axis. The interaction regions are centered at $\pm 2 \mathrm{~cm}$ from center.

(b) Magnetic field in gap center as a function of supply current. 


\section{Electric Field Pulser}

An electric fielf of several hundred volts per centimeter is needed in each interaction region in order to generate a Stark signal much larger than the background. The electric field in the two adjacent regions should be in opposite directions so that an asymetry can be calculated on a shot-to-shot basis, thereby cancelling fluctuations in the data due to intensity fluctuations in the laser. Furthermore the electric fields in the region should change sign on a shot-to-shct basis in order to cancel instrumental asymetries, and the electric field pulses must be short, both to capacitively couple the pulse into the cell, and to prevent discharges from occurring at the densities used.

An electric field pulser was designed with a fast rise and fall time to minimize the time an electric field was present in the cell. The pulser design is quite simple. A dc regulated HV supply (Hewlett Packard 6516A) charges a delay line through a $10 \mathrm{M} \Omega$ resistor. A stack of 6 SCRs (GA201 A Unitrode) holds off the voltage until a pulse synchronized with the laser causes them to conduct. The output is teminated by a $125 \Omega$ resistor and the voltage sensed across the resistor is applied to a cell electrode. The turn on time of the SCRs is several nsec and the rise time of the pulse is $6 \mathrm{nsec}$. The charging time of the delay line is very slow compared to the pulse width of 50-80 nsec (depending on the length of the delay lines) so that the pulse turns off abruptly when the delay line is discharged, with a fall time of approximately $20 \mathrm{nsec}$. A trace of the pulse shape along with a diagram of the pulser are given in Fig. IV-12. 


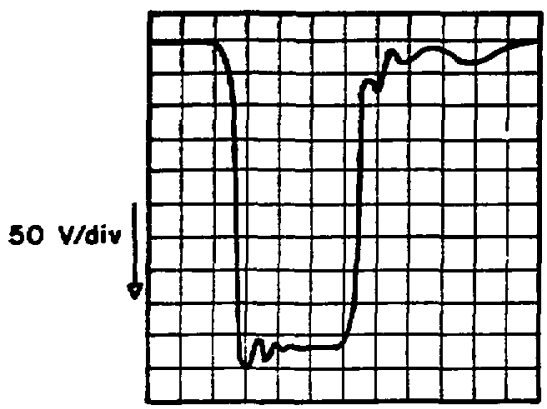

20 nsec/div $\longrightarrow$

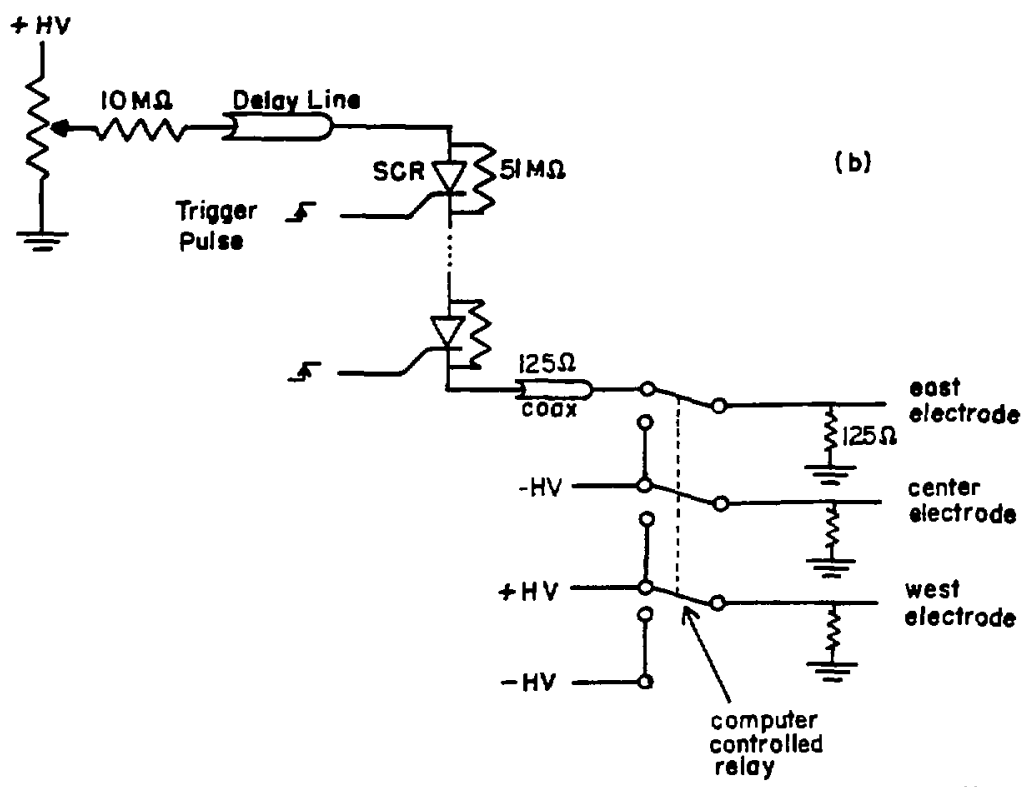

XBL $833-8857$

Fig. IV-12. (a) Oscilloscope trace of electric field pulse measured using a Textronix7904 fast scope. (b) Electric field pulser schematic. 
The pulser is made of 4 boards of 6 SCRS, each with its own delay line; two of the boards generate positive voltage pulses and the other two generate negative voltage pulses. Potentioneters allow independent fine adjustments of the magnitude of the voltage pulse from each board and computer controlled relays allow the connections between the boards and the cell electrodes to be reversed at will. The two outer pairs of electrodes are pulsed with voltage of the same sign while the center pair is chosen to have the opposite sign. The independent voltage controls on the four boards allow the regions to be independently balanced so that the reversal $+E \rightarrow-E$ is good. The Stark signal is used to make the fine adjustments in balancing the regions as will be discussed in the following chapter.

Ideally the electrode structure presents an open circuit to the voltage pulse from the electric field pulser and the entire voltage that appears across the $125 \Omega$ resistor appears on the electrode plates. This is not a good description however, and a more accurate equivalent circuit is shown in Fig. IV-13. The assumptions that the 3 band capacitances are equal, the 2 interaction region capacitances are equal, and the stray capacitances to ground are equal, are justified by independent measurements of these quantities that showed them the same to within $10 \%$ in each case. The ratio of the voltage, $v_{10}$, appearing across the electrodes in a region to the voltage $V=V_{1}=$ $v_{2}$ applied between the external capacitor bands is:

$$
\left.\frac{v_{10}}{v}\right|_{a}=1-\frac{c_{1}}{c}-\frac{3 c_{0}}{c} ; \quad v=v_{1}=v_{2} \text {. }
$$




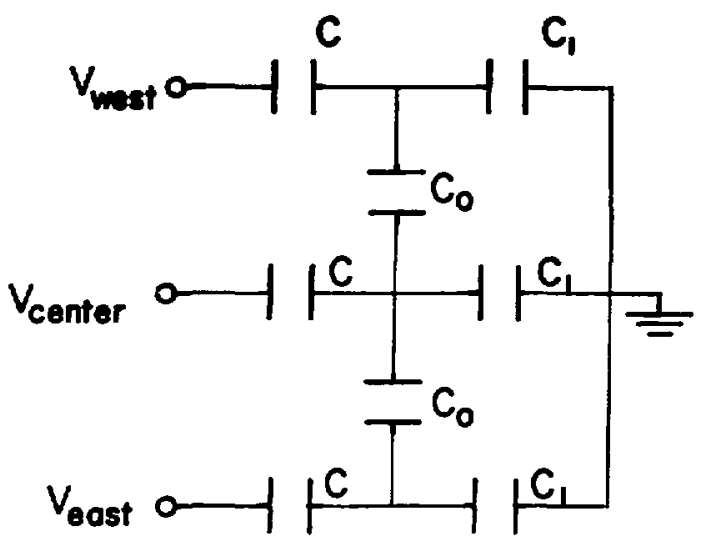

$$
V_{1}=V_{\text {oost }}-V_{\text {center }}
$$

$$
V_{2}=V_{\text {west }}-V_{\text {center }}
$$

XBL $833-8858$

Fig. IV-13. Equivalent circuit for electrode assembly. $C_{0}$ is the capacitance of the interaction region, $C$ is the capacitance of the coupling and $C_{1}$ is stray capacitance to ground. 
The ratios of the capacitances are known from direct measurements but the atoms provide an independent check. If the voltages in the two regions are made unequal by grounding one of the capacitor bands so that $v_{1}=v, v_{2}=v / 2$, then IV.2 is modified to be

$$
\left.\frac{v_{10}}{v}\right|_{b}=1-\frac{c_{1}}{c}-\frac{5}{2} \frac{c_{0}}{c} ; v_{1}=v, v_{2}=v / 2 .
$$

Experimentally, the quantity

$$
\frac{\left.v_{10}\right|_{a}}{v_{10}}=\frac{\left.\frac{v_{10}}{v}\right|_{a}}{\left.\frac{v 10}{v}\right|_{b}}
$$

can be measured by looking at the signal size in one region (which is proportional to the electric field squared) as the outer elect.. Jde in the other region is switched from a potential $V / 2$ to ground and taking the square root of the ratio of the signal sizes. Since

$$
\frac{\left.\frac{v_{10}}{V}\right|_{a}}{\left.\frac{v_{10}}{V}\right|_{b}}=1+\frac{1}{2} \frac{C_{0}}{C}
$$

and

$$
\frac{\left.\frac{v_{10}}{V}\right|_{b}-\left.\frac{v_{10}}{V}\right|_{a}}{\left.\frac{V_{10}}{V}\right|_{b}+\left.\frac{v_{10}}{V}\right|_{a}}=\frac{1}{4} \frac{c_{0}}{c}\left[1+\frac{c_{1}}{c}+\frac{11}{4} \frac{c_{0}}{C}\right] \text {, }
$$

the two equations in two unknowns can be solved to yield the following results:

$$
\begin{aligned}
& \frac{c_{1}}{c}=.13 \\
& \frac{c_{0}}{c}=.01 .
\end{aligned}
$$


This compares well with the values abtained by direct measurement of $C_{1}$ and $C$. Using the LC meter

$$
\frac{C_{1}}{c}=.15 \text {. }
$$

The direct measurements of capacitance cannot be repeated once the cell is sealed but the indirect ones can be made with high precision (a computer program is used to switch the E-field and to monitor and normalize the data to fluctuations in laser intensity) and the electric field in the region determined to $\pm 5 \%$.

4. Detection System and Data Acquisition

The $535 \mathrm{~nm}$ light from the decay of the thallium $7^{2} \mathrm{~S}_{1 / 2}$ state is detected and the magnitude of that signal is analyzed for its dependence on $w$ and the direction of $\hat{E}, \vec{E}, \vec{B}$, and $\hat{k}$. The detection of the signal proceeds in two stages. First the light from the thallium cell is collected and focussed onto the cathode of a photomultiplier tube and the resultant electron current is integrated and amplified, and secondly, the signal is digitized and stored in an LSI-11 computer where the data are binned according to the sign of $\hat{E}, \vec{E}, \vec{B}, \hat{k}$ and the frequency of the laser. The detection system is shown in Fig. IV-14.

The $535 \mathrm{~nm}$ fluorescence from each interaction region passes through the holes in the oven and heat shields and is collected by 4" f-1 Fresnel lenses, chosen because of their small spherical aberration. To protect the lenses, filters, and phototubes from the hot oven, the detection optics are enclosed in a water-cooled brass housing, re-entrant in the vacuum can, so that the optical path length from the interaction region to the first lens is just $4 "$ ". A liquid 


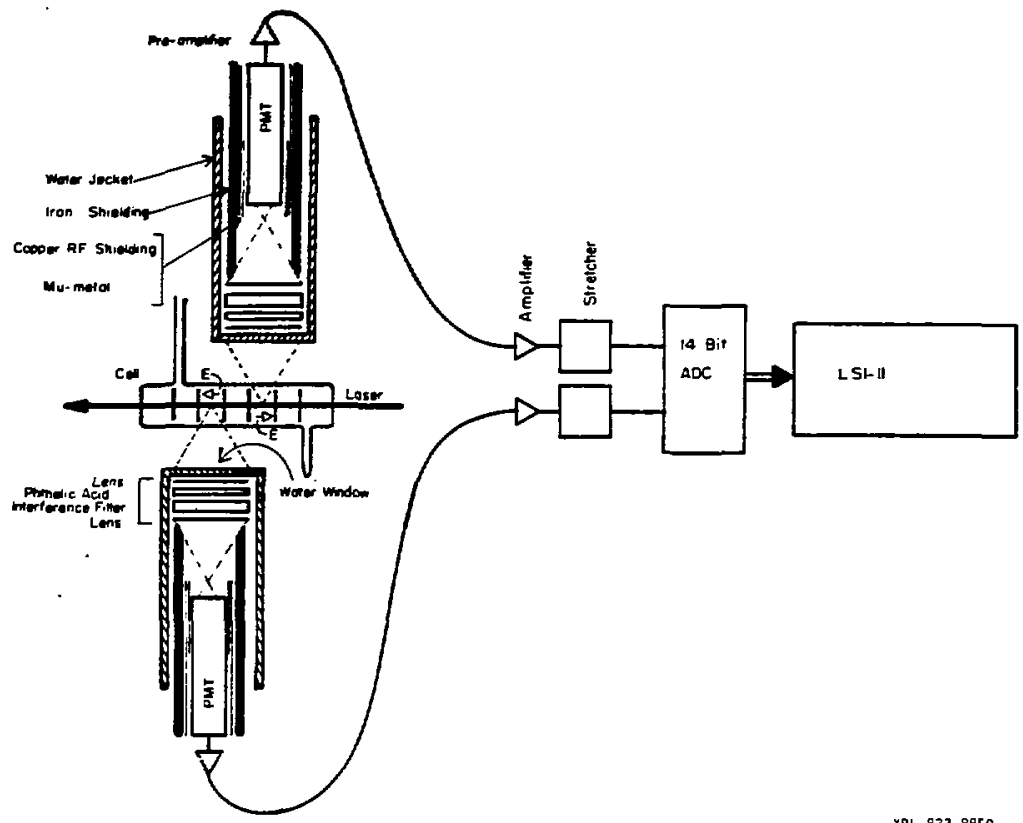

X日L $837-8859$

Fig. IV-14. Detection system (not to scale). 
filter of $12 \mathrm{gm}$ of potassium acid phthalate per liter of distilled water, 1/4" thick, placed in front of the first lens, effectively blocks any scattered laser light from the lens. 50 After the lens, the collimated light passes through a 4" diameter $535 \mathrm{~nm}$ interference filter with a $10 \mathrm{~nm}$ bandwidth, 60\% peak transmission, and is refocussed with another $4^{*} f-1$ Fresnel lens. A spatial filter at the lens focus is useful for removing some background light produced by electrons photoejected at the electrodes, but it also decreases the signal substantialiy since the interaction region is a line rather than a point source. The phototube is placed $1 "$ in back of the lens focus to prevent photocathode saturation. A $3 / 8$ " thick iron cylinder and cylindrical mu-metal shields protect the phototube from the magnetic field and a grounded copper shield protects the tube from rf broadcast from the electric field pulser and the lasers.

Approximately $10^{5}$ photoelectrons are produced during each laser pulse and the tube (EMI 9658) must be run at a low gain to prevent saturation of the last stages. The anode of the tube is capacitively coupled to a homemade change integrating preamp mounted on the base of the tube which consists of a switch-selectable RC integrator follow by a high speed buffer amplifier (National Semiconductor LHO033) designed to drive high currents. The voltage puise out of the preamp is amplified and shaped by a high rate linear amplifier (LBL 1.1\%5501) and the peak voltage held by a pulse stretcher (LBL $11 \times 9421$ ) until it is digitized by a 14 bit $A$ to $D$ converter (ADAC) and stored in the computer (LSI-11/2). For diagnostic purposes it is useful to gate the charge coming off the anode of the phototube and only accept a portion 
of the pulse. To this end, fast gates ( 2 nsec rise time) were built to go on the PMT anode using a charge-to-time converter (LeCroy QT-100C) where the ramp voltage was sensed instead of the TTL output usually used. The gates could be used to give informaton on the temporal features of the background but did not improve signal to background ratios as much as was initially hoped, because most of the background is light from the $7^{2} \mathrm{~S}_{1 / 2}$ state and has the same time features as the signal.

The signal sent to the computer is binned according to the sign of $E$ and $\theta$. In each group of 16 laser shots four shots of data are taken in each of the configurations

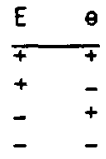

in an order assigned by the computer's random number generator. A parity asymetry is then formed by calculating

$$
\Delta_{\mathrm{Par}}=\left[\left({ }^{++}\right)-(+-)-(-+)+(++)\right] / 4
$$

where the region subtraction $\left(S_{1}-S_{2}\right) /\left(S_{1}+S_{2}\right)$, has already been made. The data are then averaged over a total of 256 laser shots and stored on disk. A total of 20 quantities are stored each 16 seconds: an average asymmetry, the signal averages for each region for each independent configuration, the average total ignal, and the sums of the squares for all these quantities so a standard deviation can be calculated. Approximately once per minute the following information is printed out: the parity averaged over 1024 shots, the standard 
deviation in the data for 16 shots, and then four diagnostic quantities $r_{E}^{1,2}$ and $\gamma_{U V}^{1,2}$ formed as follows:

$$
\begin{aligned}
& r_{E}^{1}=\frac{S_{1}(E+)-S_{1}(E-)}{S_{1}\left(E^{+}\right)+S_{1}(E-)} ; r_{U V}^{1}=\frac{S_{1}(U V+)-S_{1}(U V-)}{S_{1}(U V+)+S_{1}(U V-)} \\
& r_{E}^{2}=\frac{S_{2}(E+)-S_{2}(E-)}{S_{2}\left(E^{+}\right)+S_{2}(E-)} ; r_{U V}^{2}=\frac{S_{2}(U V+)-S_{2}(U V-)}{S_{2}\left(U V^{+}\right)+S_{2}(U V-)}
\end{aligned}
$$

where $S_{1(2)}$ refers to region $1(2)$ and $S\left(E^{+}\right)$means the data with $E^{+}$ have been averaged over UV+ and UV- and so forth. Since the signal is proportional to the electric field squared, adjusting the potentiometers of the electric field pulser to keep $r_{E}^{1}$ and $r_{E}^{2}$ very small, insures that the electric field has the same magnitude for both polarities ir each region. $Y_{U V}^{1}$ and $Y_{U V}^{2}$ serve a similar function. Keeping them small by rotating Pockels cell I slightly insures that $\theta^{+}$and $\theta-$ are symmetric about the magnetic field. These quantities typically are kept to no larger than a few parts in $10^{-3}$ in each short printout. After each short printout, the computer either changes the frequency of the laser to a line of opposite asymmetry, blocks or unblocks the mirror at the back of the can, or both. After 30 minutes, the computer prints out a summary of this data for each of the configurations, including a calculation of the asymmetry in the difference between the data taken at the different frequencies. An off-line analysis program compiles the data in larger bins. 


\section{Calibration Experiments}

There are several things in the experiment that need to be calibrated: the magnetic field, the electric field, and int frequency scanning of the Taser. It is also important to demonstrate sources of noise and verify that photon counting statistics are the main source of uncertainty in the data.

The calibration of the electric field has already been discussed in some detail. The potentials applied to the external capacitor bands are well known and are measured with a fast scope (HP7904 with P6106 probe). The output of one board in the pulser is used in the calibration. The potentiometer setting for that board is never changed and the other boards are balanced relative to it. Corrections to the value of the field must then be made to account for geometry and the effects of the capacitive coupling, as has already been discussed.

The magnetic field in the interaction region is measured directly with a Hall probe and the scanning rate of the $\mathrm{cw}$ laser is measured using an interferometer (Tropel Model 240) with a free spectral rango of $1.5 \mathrm{GHz}$. An independent method to measure boih these quantities exists by using the thallium atoms to sense frequency and magnetic field. Programs have been written to scan the laser frequency across the hyperfine structure automatically and record the signal size as a funct' sa of frequency. Data are caken with the magnet off to measure the Doppler width and then again with the magnet on to measure $\theta$ and B. In both cases the Pockels cell switches from $\theta=0^{\circ}$ to $\theta=90^{\circ}$ so that both $\Delta m_{F}=0$ and $\Delta m_{F}= \pm 1$ data are recorded. Data are 
stored on disk and then analyzed with a curve fitting program that searches parameter space for a minimum $x^{2}$. For the $B=0$ curve, the hyperfine and Doppler shift splittings and line ratios are fixed and $x^{2}$ is minimized by varying the Doppler width and the horizontal scale to check the laser scan rate. The overall intensity normalization is also allowed to vary. For the $B=0$ curves, the Doppler width and horizontal scale are fixed and $\chi^{2}$ is minimized by varying $B, \theta$ and the intensity. Both fits also fit a linear background. Figure IV-15 shows scan data and the results of a $\chi^{2}$ fit for $B=2.2 \mathrm{KG}$. There are features in the lineshape not accounted for by a simple Doppler profile that have to be understood befcre the best fit values for $B$ and $\theta$ can be taken too seriously. The açreement between fit and data is generally good, and decreasing the background will allow more accurate scans.

Because the signal is small (we want to work at the lowest possible electric field the background dilution permits in order to maximize the asymetry) it is important to consider sources of noise and how they might affect the data. The main source of fluctuatins should be counting statistics. The best way to check whether or not this is the case is to measure the fluctuations in the data as a function of the inverse of electric field and see if the relation is linear. This will then verify whether or not the fluctuations are indeed proportional to the inverse of the square root of the number of photons counted. Various $\in$ lements of the experiment have been found to contribute to the fluctuations: saturated phototubes, noise from electronics, misaligned laser, and bubbles in the water windows. These have been removed and Fig. IV-16 shows a plot of $\sigma$ vs. 1/E. The fact 


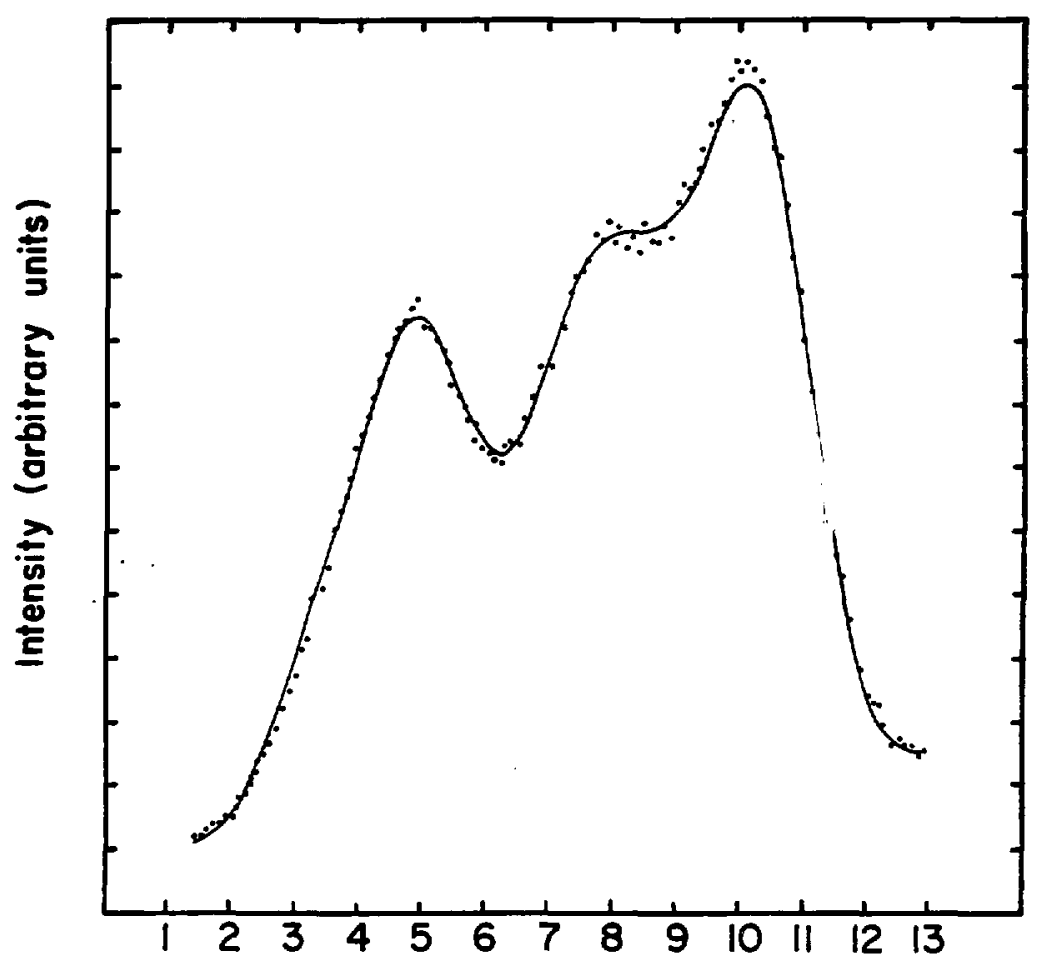

Frequency $(\mathrm{GHz})$

XBL 834-9058

Fig. IV-15. Results of a laser scan of the hyperfine structure for $B=2.2$ KGauss and $\theta=0^{\circ}$. The solid line is a fit to the data. 
that the curve does not go through the origin reflects the presence, on the level of 8 parts in $10^{-4}$ per pulse, of noise in the system contributed by electronics. Figure IV-16 includes a curve where the phototubes were at the focus of the final fresnel lens and the saturation is quite apparent. The data collection rate represents an order of magnitude improvement over previous experiments in thallium 31 and will allow measurements with a precision of 1 part in $10^{5}$ to be made in a few hours. 


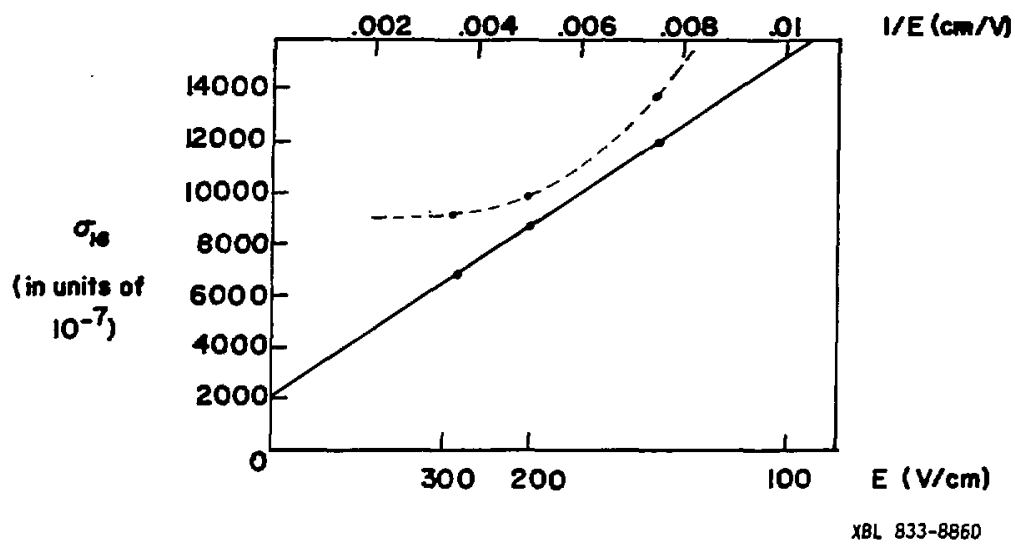

Fig. IV-16. Standard deviation for 16 laser shots versus the inverse of the electric field for phototubes at the focus (dotted line) and 1 in. behind focus (solid line) of final fresnel lens. 


\section{RESULTS}

This experiment cannot claim any significant limits on or measurement of parity nonconservation in atomic thallium. Because of the Targe asymmetry in the background thought to be due to electrons, (as explained in Chapter III), an asymmetry in the signai can only be measured with a precision of a few parts in $10^{5}$. The expected asymmetry due to weak neutral current effects is approximately one part in $10^{5}$ so the existing limit is not very meaningful. Increased statistics could improve this limit somewhat, but uncertainties in elimination of the background asymetry would prevent a precise measurement. Instead the experiment is being modified to eliminate the background and the parity measurement will wait until the changes are completed. Nevertheless, investigation of the possible systematic asymetries associated with misalignments in the experiment, as listed in Fig. III -4 , have yielded interesting results. Limits exist for all of these possible systematics except the $a_{1} a_{3}$ interference. These limits will be improved easily when the background is reduced, but they all put the size of possible false asymmetries significantly below the expected parity asymetry. In this chapter the methods for measuring these false parity effects will be described in detail and the results of these measurements will be presented.

\section{On-Line Limits}

While the experiment is accumulating parity data, two quantities can be measured concurrently: $\Delta E_{y}$ and $\theta_{x}$. This is done using $\gamma_{E}^{1,2}$ and $\gamma_{U V}^{1,2}$ as defined in (IV.9). 
The leading term in the transition probability is

$$
\begin{cases}B_{2}{ }^{2} \sin ^{2} \theta & \Delta m_{F}=0 \\ B_{2}{ }^{2} \cos ^{2} \theta & \Delta m_{F}= \pm 1 .\end{cases}
$$

Using IV.9 and recalling that

$$
B_{2}=B\left(E_{\text {oy }}+\Delta E_{y}\right)
$$

where $E_{o y}$ is the reversing and $\Delta E_{y}$ the non-reversing $y$ component of the electric field, then

$$
Y_{E}=\frac{S\left(E_{+}\right)-S\left(E_{-}\right)}{S\left(E^{+}\right)+S\left(E_{-}\right)}=\frac{2 \Delta E_{y}}{E_{D y}} .
$$

The quantity $\gamma_{E}$ is computed separately for the two regions. The sizes of the electric field for $\mathrm{E}^{+}$and $\mathrm{E}-$ can be independently adjusted for the regions individually in order to minimize $\gamma_{E}$. It is possible during a run to keep

$$
\left|\Delta E_{y}\right|<5 \times 10^{-4}\left|E_{o y}\right|
$$

for each region. This value is combined with other data to put a limit on the $\mathrm{B}_{2}{ }^{2} \mathrm{\theta}_{\mathrm{x}}$ interference term which gives an asymmetry of

$$
{ }^{\theta} x \text { alse }=\frac{4 \Delta E_{y^{\theta} x}}{E_{o y}} \text {. }
$$

(Note that $\Delta E_{y}$ does not enter into the asymmetries induced by the other interference terms containing a factor of $\beta_{2}$ except for effects which are of third order and thus negligible.)

Now consider $\gamma_{U V}$ which is also measured on-line. Recall that

$$
Y_{U V}=\frac{S(U V+)-S(U V-)}{S(U V+)+S(U V-)}
$$

where now more than the leading term in (V.1) must be kept in computing $r_{U V}$. Returning to the expressions for the total 
transition probability one finds:

$$
r_{U Y}= \begin{cases}-2 \theta_{x} \cot \theta & \Delta m_{F}=0 \\ +2 \theta_{x} \tan \bullet & \Delta m_{F}= \pm 1 .\end{cases}
$$

$\gamma_{U V}$ depends on the misalignment in the $x$ direction of the magnetic field as a result of the definition of the $z$-axis as the axis that bisects the angle between $\theta^{+}$and $\theta-$. What $\gamma_{\mathrm{UV}}$ allows then is the precise alignment of the laser polarization with respect to the magnetic field. The rotation angle, $B$, of PCI (see Fig. IV-3) is adjusted to minimize $\gamma_{U V}$ and thus insure that $\theta_{x}=\frac{B_{x}}{B_{z}}$ is small. It is possible during a run to keep

$$
Y_{U V}<2 \times 10^{-3}
$$

which correspond to the following limits on $\theta_{x}$ :

$$
\begin{aligned}
\left|\theta_{x}\right| & <7 \times 10^{-4} & \Delta m_{F} & =0 \\
& <1.4 \times 10^{-3} & \Delta m_{F} & = \pm 1
\end{aligned}
$$

for each region. Thus we have the limit for each region independentiy that

$$
\stackrel{\theta}{\Delta} \underset{\text { false }}{ }<2 \times 10^{-6} \text {. }
$$

This limit alone, extracted from several hours of data, is quite good. However the region subtraction has yet to be taken advantage of. If If $\theta_{x}$ and $\frac{\Delta E_{y}}{E_{y}}$ are the same in both regions they cannot contribute a false parity since the signals in the two regions are subtracted to calculate an asymmetry. Both $\theta_{x}$ and $\frac{\Delta E_{y}}{E_{y}}$ are strongly correlated in the regions so making that subtraction gives

$$
\stackrel{\theta}{\Delta_{\text {false }}}<1.2 \times 10^{-7} \text {. }
$$


The contribution to the assymmetry due to the $s_{2}^{2} \theta$ interference is negligible.

\section{Circular Polarization Measurements}

In order to investigate interference terms involving $a_{3}$, wich arise from the component of the electric field along the 2 axis, an auxiliary experiment is performed using circularly polarized (CP) incident laser light. To make CP light Pockels cell II is removed and Pockels cell $I$ is aligned so that the effective fast axis is at $45^{\circ}$ to the incident laser polarization. The voltage on the Pockels cell is then adjusted to produce quarter-wave retardation. Changing the sign of the high voltage reverses the helicity of the light.

The transition amplitudes for $C P$ light are easy to construct, and Fig. III-2, with the replacement

$$
\begin{aligned}
\cos \theta & \rightarrow \frac{1}{\sqrt{2}} \\
\left(1+\delta_{\varepsilon}\right) \sin \theta & \rightarrow \pm \frac{i}{\sqrt{2}},
\end{aligned}
$$

is still valid. Keeping only the highest order interference term in the transition probability gives

$$
\begin{aligned}
& T_{i}\left(\Delta m_{F}=0\right)=\frac{1}{2} a_{i} \beta_{2}^{2} \pm b_{j} \beta_{2} a_{3} \\
& T_{i}\left(\Delta m_{F}= \pm 1\right)=\frac{1}{2} a_{i} \beta_{2}^{2} \pm b_{i}\left(\beta_{2} \beta_{3}+\theta_{y} \theta_{2}^{2}\right)
\end{aligned}
$$

where $a_{j}$ is the coefficient of the leading term listed in Fig. III-4,

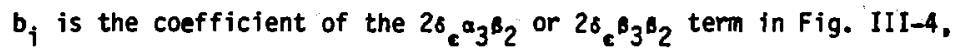
and \pm refers to $*$ helicity incident laser light. For data taken with CP light, 


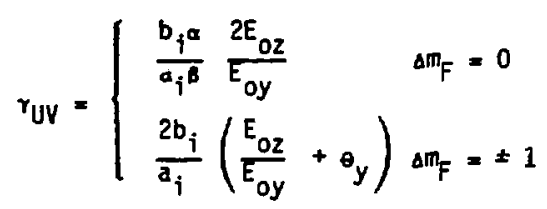

in each region. $\gamma_{E}$ is the same as for linearly polarized light but the leading teirm in the parity channel where the electric field, UV, and region substractions have been made is:

$$
\Delta_{f a l s e}^{C P}= \begin{cases}\frac{b_{i}}{a_{i}} \frac{a}{B} \frac{2 \Delta E_{2}}{E_{o y}} & \Delta m_{F}=0 \\ \frac{b_{i}}{a_{i}} \frac{2 \Delta E_{2}}{E_{o y}} & \Delta m_{F}= \pm 1 .\end{cases}
$$

Apart from the factor of $\varepsilon_{\varepsilon}$ this is exactly the contribution that the $\delta_{\varepsilon}{ }_{2} a_{3}$ or $\delta_{\varepsilon} \dot{B}_{2} B_{3}$ interference terms make to the true parity asymetry when the experiment is performed with linearly polarized light. In the actual experiment $\delta_{E}$ is very small since the quality of the linear polarization is so good, but here $C P$ has enhanced these interference terms by more than an order of magnitude.

Approximately 4 hours of data were taken with CP Tight on two transitions. Both the main $\Delta \mathrm{m}_{F}=0$ transition which is a combination of the $|1,1\rangle \rightarrow|1,1\rangle,|1,-1\rangle \rightarrow|1,-1\rangle$ and $|+\rangle \rightarrow|+\rangle$ ines, and the most prominent $\Delta m_{F}= \pm 1$ transition, $|1,-1\rangle \rightarrow|+\rangle$, were used. On the $\Delta m_{F}=0$ transition, che effect cancels in the $|1,1\rangle \Rightarrow|1,1\rangle$ and $|1,-1\rangle \rightarrow|1,-1\rangle$ lines and only enters at half strength through the $|+\rangle \rightarrow|+\rangle$ line which is almost $1 \mathrm{GHz}$ off resonance. The ful $\}$ effect enters in the $|1,-1\rangle \rightarrow$ It> line undiluced but the line is weaker and the statistical precision not as good. From the parity data where all the subtractions were made and $r_{y v}$ where the data were averaged over electric field and the 
region subtraction has been included, the following 1 imits were extracted:

$$
\begin{aligned}
& \left|\Delta E_{z}\right|=(1.3 \pm .4) \times 10^{-3}\left|E_{o y}\right| \\
& \left|E_{o z}\right|=(2 \pm .7) \times 10^{-3}\left|E_{o y}\right| .
\end{aligned}
$$

Limits can now be placed on two additional interference terms:

${ }^{5} \varepsilon_{2} \alpha_{3}$ and $a_{3}{ }^{M}$.

The $\delta_{\varepsilon_{2}{ }^{\alpha} \alpha_{3}}$ term contributes a false parity asymetry to the linear polarization PNC experiment of

$$
\Delta_{\text {false }}^{\delta}=\frac{{ }^{2 \delta_{\varepsilon} \Delta E_{z}}}{E_{\text {oy }}} \frac{\alpha}{B}\left(\Delta m_{F}=0\right) .
$$

The quality of the linear polarization is very good and the measured limit on $\delta$ is

$$
\begin{aligned}
& \delta_{\varepsilon}^{2}<10^{-3} \\
& \delta_{\varepsilon}<.032
\end{aligned}
$$

This combined with (V.15) yields

$$
\Delta_{\text {false }}^{\delta}<8 \times 10^{-5},\left(\Delta m_{F}=0\right) .
$$

The $\mathrm{Ma}_{3}$ term contributes a false parity asymetry of

$$
\Delta_{\text {false }}^{M}=\frac{2 \alpha M E_{O Z}}{B^{2} E_{o y}^{2}}\left(\Delta m_{F}=0\right) .
$$

From (V.15) and the values of $M / B$ from Chapter II, one gets:

$$
\begin{aligned}
\Delta_{\text {false }}^{M} & =6 \times 10^{-3} \times \frac{1}{E}(\mathrm{~V} / \mathrm{cm})\left(\Delta m_{F}=0\right) \\
& =2.4 \times 10^{-5} \quad \text { for } E=250 \mathrm{~V} / \mathrm{cm} .
\end{aligned}
$$

As they stand (V.18) and (V.20) are not satisfactory limits, but several factors allow these limits to be improved so that these false asymmetries can be distinguished from the true effect. 
When the direction of the magnetic field is changed, both of the above false asymmetries reverse sign but the asymmetry induced by neutral current effects does not change. After the magnetic field subtraction has been made, then

$$
\begin{aligned}
& \Delta_{\text {false }}^{\delta}<5 \times 10^{-6} \\
& \Delta_{\text {false }}^{M}<1.5 \times 10^{-6} .
\end{aligned}
$$

These are limits that can be improved as increased statistics verify the quality of the subtraction. These two false asymmetries also reverse sign when the direction of the laser beam is reversed, whereas the true parity asymmetry is independent of $\hat{k}$. It is expected that this subtraction will further reduce the limits in ( $V .21)$ by a factor of 4 .

For the $\Delta m_{F}= \pm 1$ transitions this is the best that can be done and it is possible that the $\Delta m_{F}= \pm 1$ data may be limited by the uncertainty in the contribution from the $\delta_{\varepsilon} E_{y} E_{z}$ interference. (Actually the limits quoted in (V.21) are a little smaller for the $\Delta m_{F}= \pm 1$ transition where the factor $\alpha / \beta$ is replaced by unity). In the $\Delta m_{F}=0$ transition that we choose to work on however, the false asymmetry is further reduced by a factor of 8 since these interference terms cancel in the $|1,1\rangle>|1,1\rangle$ and $|1,-1\rangle \rightarrow|1,-1\rangle$ transitions as mentioned previously. The contribution from the $|+\rangle|+|+$ line is supressed a factor of 2 because it is off resonance and both terms in (v.21) become negligible. 
3. $\theta_{y}$ and 700 Gauss Measurements

The circular polarization measurements and the data accumulated while running the experiment have set limits on the asymmetries induced by three of the interference terms jisted in Fig. III-4. To set jimits on the remaining two terms requires two additional experiments.

The interference term proportional to $B_{1} B_{2}{ }^{\theta} y$ contributes an asymmetry of the form

$$
\stackrel{\theta_{\mathrm{y}}^{y}}{\Delta_{\text {false }}}=\frac{2 \Delta E_{x^{\theta} y}}{E_{y}} .
$$

This is a troublesome term because $\Delta_{\text {false }}^{\theta_{y}}$ changes sign and magnitude in the same was as the true parity asymmetry as the hyperfine structure is scanned, and it behaves the same way under reversal of $B$ and $k$. In order to put a limit on this term, it is first enhanced by rotating the entire vacuum system inside the magnet gap. If the laser beam is then realigned so that it goes through the cell in the same fashion as before, $\Delta E_{x}$ will have been unchanged but $\theta_{y}$ will have been greatly increased. There is sufficient room in the magnet gap to rotate the vacuum can $\neq 10 \mathrm{mrad}$ and $i t$ is aligned to within I mrad of center so $e_{y}$ is approximately 10 times greater with the can tilted.

Data are taken with the can tilted to botn $+10 \mathrm{mrad}$ and $-10 \mathrm{mrad}$ and the parity channel is examined for any evidence of an asymmetry that changes sign with the can angle. Que to the large asymetry in the background, data were taken on both the main $\Delta \mathrm{m}_{F}=0$ transition and on $a \Delta m_{F}=+1$ transition and combined to el iminate background effects. Figure $V-I$ shows the data from which we can coriclude: 


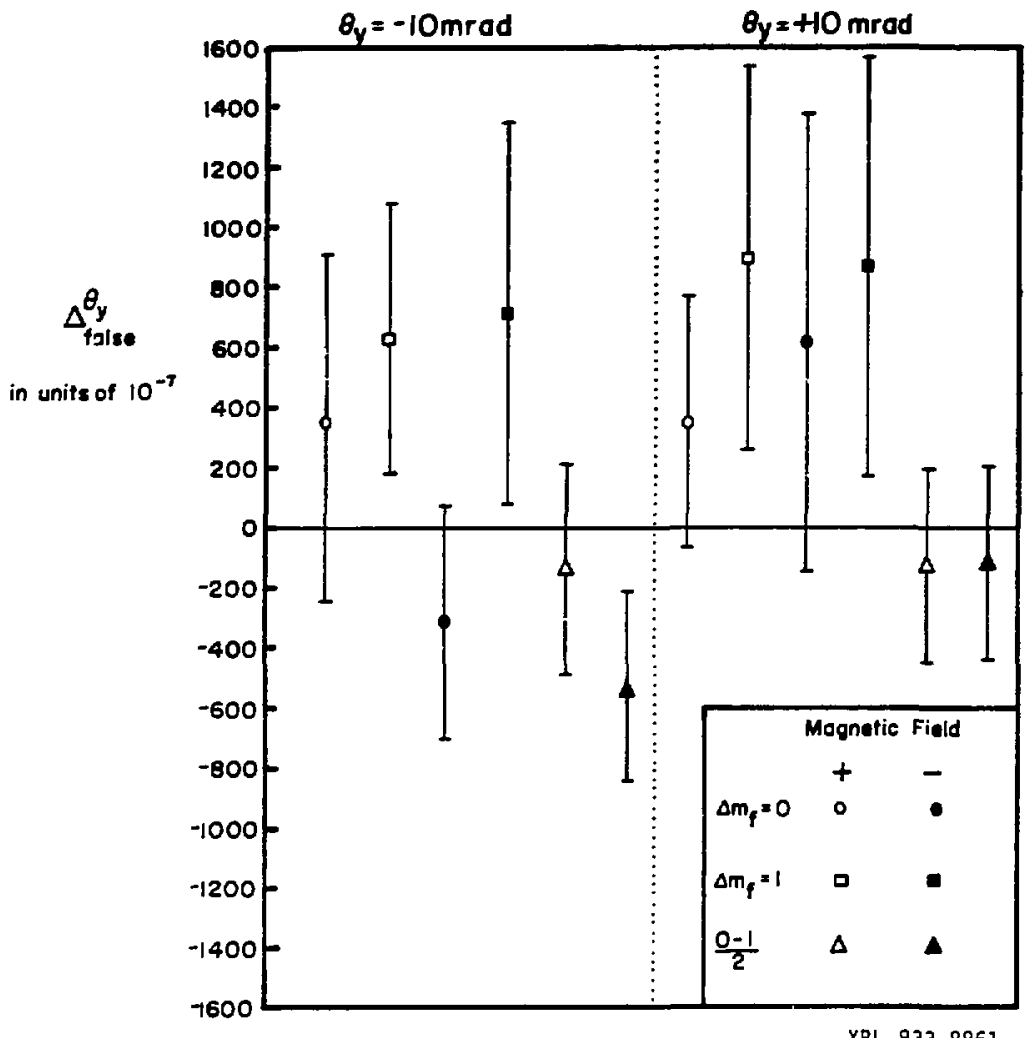

XBL 833-8861

Fig. V-1. Asymmetry in parity channel with can tilted 10 mrad. Data are shown for two transitions: $|1,1\rangle \rightarrow|1,1\rangle$, $|+>\rightarrow|+\rangle,|1,-1\rangle \rightarrow|1,-1\rangle \Delta \mathrm{mF}=0$ line and $|1-1\rangle+|+\rangle \Delta \mathrm{mF}=$ +1 ine. $0-1 / 2$ is the asymmetry in the difference between the two lines where the subtraction is used to remove any asymmetries in the background. Data are shown for $B= \pm 3.6$ Kgauss. 


$$
\left|\Delta E_{x}\right|<1 \times 10^{-3}\left|E_{o y}\right|
$$

where the limit is purely statistical. Therefore, with the cell centered in the magnet gap, the limit is

$$
\stackrel{\theta y}{\Delta \text { false }}<2 \times 10^{-6} .
$$

This 1 imit can be improved when removal of the asymmetry in the background makes a more precise measurement possible.

The last interference term in the transition amplitude that can produce a false asymmetry is the term resulting from the components or the misaligned electric field: $a_{1} a_{3}$ and $\theta_{1} b_{3}$ for $\Delta m_{F}=0$ and $\Delta m_{F}= \pm 1$ respectively. These terms induce an asymmetry of the form

$$
\Delta_{f a l s e}^{x z}= \begin{cases}\frac{2 a^{2}}{B^{2}} \frac{\left(E_{o x} \Delta E_{Z}+E_{o Z} E_{X}\right)}{E_{o y}^{2}} & \Delta m_{F}=0 \\ \frac{2\left(E_{o x} \Delta E_{Z}+E_{o z} \Delta E_{x}\right)}{E_{o y}^{2}} & \Delta m_{F}= \pm 1\end{cases}
$$

It has already been demonstrated that $E_{0 z}, \Delta E_{z}$, and $\Delta E_{x}$ can be independently determined but there is no independent way that $E_{o x}$ can be measured. This effect could be substantial, since if $E_{o x}$ is roughly the size of $E_{0 z}$ then (V.15) and (V.23) combine to give

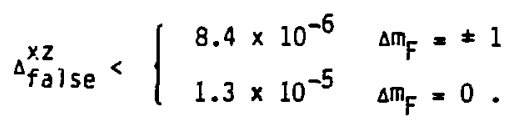

These are clearly unsatisfactory limits, and there is no guarantee that $E_{o x}$ is not iarger than $E_{0 z}$ though the geometry prevents it from being mure than a factor of 2-3 bigger. This false asymmetry behaves exactly like the true parity asymmetry as the magnetic field 
and laser direction are reversed, and it has the same behavior on the different hyperfine transitions. Fortunately, a separate experiment allows the measurement $s_{f a l s e}^{x z}$ directly so that it can be corrected for if necessary.

At a magnetic field of approximately 700 Gauss, the Zeeman splittings of the lines are negligible and the only transitions that can be resolved are

$$
\begin{aligned}
& 6^{2} \mathrm{P}_{1 / 2}, F=0 \rightarrow 7^{2} \mathrm{P}_{1 / 2}, F=0 \\
& 6^{2} \mathrm{P}_{1 / 2}, F=0 \rightarrow 7^{2} \mathrm{P}_{1 / 2}, F=1 \\
& 6^{2} \mathrm{P}_{1 / 2}, F=1 \rightarrow 7^{2} \mathrm{p}_{1 / 2}, F=0 \\
& 6^{2} \mathrm{P}_{1 / 2}, F=1 \rightarrow 7^{2} \mathrm{P}_{1 / 2}, F=1 .
\end{aligned}
$$

The transition nrobability for the $1 \rightarrow 1$ transition can be constructed by summing the appropriate entries from Fig. III-2 which gives

$$
T_{1 \rightarrow 1}=2 B_{2}^{2}+\left(6 a_{1} a_{3}-4 \beta_{1} B_{3}\right) \sin \theta \cos \theta
$$

where only the leading order term and leading interference term have been kept. When the lines overlap, the parity nonconserving interference cancels so that in the parity channel where electric field, UV, and region subtractions have been made, the only asymmetry that can contribute is

$$
\begin{aligned}
\Delta_{f a I s e}^{700} & =\frac{3 a^{2}-2 B^{2}}{s^{2}} \frac{\left(E_{0 x} \Delta E_{z}+E_{0 z} \Delta E_{x}\right)}{E_{o y}^{2}} \\
& =\frac{2.7\left(E_{o x} \Delta E_{z}+E_{o z} \Delta E_{x}\right)}{E_{o y}^{2}} .
\end{aligned}
$$

Apart from numerical factors, this is identical to (v.25). This quantity, $\Delta_{f a l s e}^{700}$, must be measured with the same precision as the true 
parity asymetry and then the data must be corrected for it. Due to the small size of this term and because of the precision with which it must be measured, the large asymmetry in the background has prevented a meaningful limit from being placed on it so far.

When the true parity asymmetry is measured, data are taken at two different frequencies, where the asymetry is opposite in sign but the ratio of signal to background is the same, so that any residual background asymmetries can be canceled. In the 700 Gauss data, this is not possible because the different $m_{F}$ transitions are not resolved. Instead, data are taken on and off resonance with a suitable correction applied for the signal to background ratio, and the data are subtracted to remove any possible background asymmetry present in $\Delta_{\mathrm{false}}^{700}$.

The choice of 700 Gauss for this auxiliary experiment was somewhat arbitrary and data will be taken at several fields to insure the independence of (V.29) from the value of the field (apart from known contributions due to the line splittings). Since the overlap of the hyperfine lines supresses the true PNC asymmetry relative to the $E_{x} E_{2}$ interference, a small magnetic field is used. Zero fieid was not chosen however, because the background due to electrons is substantially different at zero field and at several hundred Gauss, where the electron orbits are already only on the order of $1 \mathrm{~mm}$. The change in the size and dependence on $E$ of the background as $B$ is increased from 0 to 100 Gauss is dramatic but no change is seen as $B$ continues to increase. Thus any possible contribution that electrons in the cell might be making to $E_{o x}, E_{o z}, \Delta E_{x}$, or $\Delta E_{z}$ should not be changing as the 


\section{8}

as the magnetic field is increased. This will be verified directly by measuring ${ }^{700} \mathrm{false}$ at several magnetic fields.

Figure $Y-2$ is a summary of the existing limits on the possible false asymmetries for this experiment. With the new cell they will all be remeasured and, it is hoped, great?y improved. Only systematics that are the product of up to two small quantities have been included in Fig. V-2. All quantities third order and higher have been examined and discarded because all have been found to contribute less than $10^{-7}$ to the total asymetry.

The goal of this experiment is to reduce the uncertainties in the terms in $\mathrm{V}-2$ to the $10^{-6}$ level. It would be overly optimistic to hope that the terms themselves would all contribute less than one part in $10^{-6}$ to the asymmetry, but if they can be measured with sufficient precision, they can be reliably corrected for. The corrections that are ultimately made to the data in this experiment will be very different from the corrections made in previous atomic physics experiments of this type, 29,31 (in particular we avoid substantial corrections due to imperfect cancellation of an M1 asymmetry), although the spirit of the analysis is quite similar. It is unlikely that the investigation of PNC in $\mathrm{Tl}$ will be persued in the near future below the $10-15 \%$ level of uncertainty that this experiment hopes to achieve. At that point the uncertainties in the atomic physics are much greater than the experimental uncertainties and there is not much hope at this time that the theoretical situation can be greatly improved. 


\section{Limits on Asymetries}

$$
\begin{aligned}
& \Delta_{\text {parity }}=\frac{2 U}{\mathrm{GE} \text { oy }}<3 \times 10^{-5} \\
& \Delta_{\text {false }}^{x z}=\frac{2 a^{2}}{B^{2}} \frac{\left(E_{o x} \Delta E_{z}+E_{o z} \Delta E_{x}\right)}{E_{o y}^{2}}<3 \times 10^{-5} \\
& \Delta \Delta_{\text {false }}^{M}=\frac{2 a M E_{0 Z}}{B^{2} E_{\text {oy }}^{2}}<1.5 \times 10^{-7} \Delta \mathrm{m} F=0 \\
& \left(<1.2 \times 10^{-6} \Delta \mathrm{m}_{F}= \pm 1\right) \\
& \underset{\Delta_{\text {false }}^{\theta_{y}}}{\theta_{y}}=\frac{2 \Delta E_{x}{ }^{\theta} y}{E_{y}}<2 \times 10^{-6} \\
& \Delta_{\text {fatse }}^{\theta_{x}}=\frac{2 \Delta E_{y^{\theta} x}}{E_{\text {oy }}}<1.2 \times 10^{-7} \\
& \Delta \Delta_{\text {false }}^{\delta}=\frac{2 \delta_{e} \Delta E_{Z}}{E_{\text {oy }}} \frac{a}{B}<5 \times 10^{-7} \Delta m_{F}=0 \\
& \left(<4 \times 10^{-0} \Delta m_{F}= \pm 1\right)
\end{aligned}
$$

Fig. V-2. Measured limits on systematic effects. Unless otherwise indicated, the expressions shown are valid for $\Delta m_{F}=0$ lines. Expressions for $\Delta \mathrm{m}_{F}= \pm 1$ require replacement of factors of $\frac{\alpha}{\beta}$ by 1 . 


\section{ACKNOWLEDGEMENTS}

This work benefitted enormously from the contributions of many members of the Berkeley physics community - students, faculty, and staff - who have been very generous with their scientific and moral support. I particularly wish to thank Dane Anderberg who built the thallium cells and Frank Lopez who did much of the machining for the vacuum system. I am also much indebted to Steven Chu who introduced me to experimental physics in general and lasers in particular.

I was fortunate to have Eugene Commins as my advisor for this thesis. He contributed ideas, help, and encouragement throughout. I have enjoyed learning from him and I have appreciated his patience and his confidence in me.

I gratefully acknowledge support from N.S.F. and from the Bell Telephone Laboratory G.R.P.W. program. This work was supported by the Director, Office of Energy Research, Office of Basic Energy Sciences, Chemical Sciences Division of the U. S. Department of Energy under Contract No. DE-ACO3-76SF00098. 


\section{REFERENCES}

1. Steven Weinberg, Phys, Rev. Lett. 19, 1264 (1967).

2. A. Salam, in Elementary Particle Physics: Relativistic Groups and Analyticity (Nobel Symposium No. 8), edited by N. Svartholm (Almqvist and Wikse11, Stockholm, 1968), D. 367.

3. S. Weinberg, Rev. Mod. Phys. 46, 255 (1974), and references therein.

4. E. Commins, University of California Course on Gauge Theories (unpublished) 1978; E. Commins and P. Bucksbaum, Weak Interactions of Ouarks and Leptons, (Cambridge University Press, 1983).

5. Jihn E. Kim, Faul Langacker, M. Levine, and H. H. Hilliams, Rev. Mod. Phys. 53, 211 (1981).

6. Pham Quang Hung and J. J. Sakurai, Phys. Lett. 88B, 91 (1979).

7. A. Sirlin and W. J. Marciano, New York University preprint NYO/TR3/81.

8. C. Y. Prescott et al., Phys. Lett. $\underline{77 B}, 347$ (1978); Phys. Lett. 84B, 524 (1979).

9. C. H. Llewellyn Smith and J. F. Wheater, Phys. Lett. 1058, 486 (1981).

10. R. Brandelick et al., Phys. Lett. 117B, 365 (1982); B. Adeva et al., Phys. Rev. Lett. $\underline{48}, 1701$ (1982); W. Bartell et al., Phys.

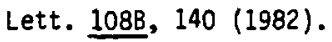

11. G. Arnison et al., Phys. Lett. 122B, 103 (1983); M. Banner et a1., Phys. Lett. 122B, 476 (1983).

12. E. D. Commins and P. H. Bucksbaum, Annu. Rev. Nucl. Part. Sci., 30, $1(1980)$. 
13. M. A. Bouchiat and C. Bouchiat, J. Phys. (Paris) 35, 899 (1974); 36, 493 (1975).

14. M. A. Bouchiat and C. C. Bouchiat, Phys. Lett. 48B, 111 (1974).

15. I. B. Khriplovich, Pis'ma Zh. Eksp. Theor. Fiz. 20,686 (1974) [JETP Lett. 20, 315 (1974)].

16. L. L. Lewis, J. H. Hollister, D. C. Soreide, E. G. Lindah1, and E. N. Fortson, Phys. Rev. Lett. 39,795 (1977).

17. P. E. G. Baird, M. W. S. M. Brimicombe, R. G. Hunt, G. J. Roberts, P. G. H. Sandars, and D. N. Stacey, Phys. Rev. Lett. 39,798 (1977).

18. L. M. Barkov and M. S. Zolotorev, Pis'ma Zh. Eksp. Teor. Fiz. 27, 379 (1978) [JETP Lett. 27, 356 (1978)].

19. L. M. Barkov and M. S. Zolotorev, Phys. Lett. 855, 308 (1979).

20. Yu. V. Bogdanov, I. I. Sobel'man, V. N. Sorok in, and I. I. Struk, Pis'ma Zh. Eksp. Teor. Fiz. 31, 556 (1980) [JETP Lett. 31, 522 (1980)].

21. J. H. Hollister, G. R. Apperson, L. L. Lewis, T. P. Emmons, T. G. Vold, and E. N. Fortson, Phys. Rev. Lett. 46, 643 (1981).

22. M. W. S. M. Bimicombe, C. E. Loving, and P. G. H. Sandars, J. Phys. B 9, L237 (1976); M. J. Harris, C. E. Loving, and P. G. H. Sandars, J. Phys. B 11 , L749 (1978).

23. V. N. Novikov, 0. P. Sushkov, and I. B. Khriplovich, Zh. Eksp. Teor. Fiz. 71, 1665 (1976) [Sov. Phys. JETP 44, 872 (1976)].

24. Ernest M. Henley and Lawrence Wilets, Phys. Rev. A 14, 1411 (1976); E. M. Henley, M. Klapisch, and L. Wilets, Phys. Rev. Lett. 39, 994 (1977). 
25. Steven L. Carter and Hugh P. Kelly, Phys. Rev. Lett. 42, 966 (1979).

26. A.-M. Martensson, E. M. Henley, and L. Wilets, Phys. Rev. A 24, 308 (1981).

27. P. G. H. Sandars, Phys. Scr. 21,284 (1980).

28. J. Hoffnagle, L. Ph. Rocsch, V. L. Telegdi, A. Weis, and A. Zehnder, Phys. Lett. 85A, 143 (1981).

29. M. A. Bouchiat, J. Guena, L. Hunter, and L. Pottier, Phys. Lett. $117 \mathrm{~B}, 358$ (1982).

30. S. L. Gilbert, R. N. Watts, and C. E. Wieman, Phys. Rev. A 27, 581 (1983).

31. P. Bucksbaum, E. Commins, and L. Hunter, Phys. Rev. Lett. 46,640 (1981); Phys. Rev. 0 24, 1134 (1981).

32. C. E. Loving and P. G. H. Sandars, J. Phys. B $\underline{8}$, L336 (1975).

33. E. M. Henley and W.-Y. P. Hwang, Phys. Lett. 88B, 349 (1979).

34. David V. Meuffer and Eugene D. Commins, Phys. Rev. A 16,1760 (1977).

35. C. Bouchiat, C. A. Piketty and D. Pignon, Ecole Normale Superiere preprint LPTENS $82 / 34$.

36. B. P. Das, J. fadriessen, Mina Vajed-Samii, S. N. Ray, and T. P. Das, Phys. Rev. Lett. 49,32 (1982); B. P. Das et al., to be published.

37. R. W. Dunford, R. R. Lewis, and W. L. Williams, Phys. Rev. A $\underline{18}$, 2421 (1978).

38. L. P. Lévy and W. L. Williams, Phys. Rev. Lett $\underline{48}, 607$ (1982). 
39. David Y. Meuffer and Eugene D. Commins, Phys. Rev. A 16, 844 (1977).

40. 0. P. Sushkov, V. V. Flambaum, and 1. B. Khriplovich, Pis'ma $2 h$. Eksp. Teor. Fiz. 24, 502 (1976) [JETP Lett. 24, 460 (1976)].

41. C. Moore, Atomic Energy Levels, Vol. III, (Nat. Bureau of Standards, 1952).

42. A. Flusberg, T. Mossberg, and S. R. Hartmann, Phys. Rev. A 14 , 2146 (1976).

43. S. Chu, E. D. Commins, and R. Conti, Phys, Lett. 60A, 96 (1977).

44. L. Hunter, E. Commins, and L. Roesch, Phys. Rev. A 25, 885 (1982).

45. Alan Gallagher and Allen Lurio, Phys. Rev. 136, A87 (1964).

46. M. A. Bouchiat and L. Pottier, Proceedings of the Work shop on Neutral Current Interactions in Atoms, Cargése (1979), edited by W. Williams

47. E. D. Commins, private communication, 1979.

48. M. A. Bouchiat, J. Guenna and L. Pottier, J. Phys. Lett. (Paris) 41, L-299 (1980).

49. S. Chu, Ph. D. Thesis, Lawrence Berkeley Laboratory report LBL-5731 (unpublished).

50. M. Kasha, J. Opt. Soc. Am. 38, 929 (1948).

51. R. H. Fowler, Phys. Rev. 38, 45 (1931).

52. A. B. Cardwe11, Phys. Rev, 47, 628 (1935).

53. G. N. Glasoe, Phys. Rev. 38, 1490 (1931).

54. Peris Drell and Steven Chu, Opt. Comm. 28, 343 (1979). 
55. A. N. Nesmayonov, Vapor Pressure of the Chemical Elements, (Elsevier, Amsterdam, 1963).

56. P. Bucksbaum, Ph. D. Thesis, Lawrince Berkeiey Laboratory report LBL-11641 (unpublished).

57. Amer. Institute of Physics Handbook, ed. D. E. Gray, (McGraw-Hi11, New York, 1963). 